\title{
Reconfigurable Band Rejection Frequency Selective Structures
}

\author{
Dissertation \\ Presented in Partial Fulfillment of the Requirements for the Degree Doctor of Philosophy \\ in the Graduate School of The Ohio State University \\ By \\ Jeffrey Scott Kula \\ Graduate Program in Electrical and Computer Engineering \\ The Ohio State University \\ 2019 \\ Dissertation Committee \\ John L. Volakis, Advisor \\ Niru K. Nahar, Co-Advisor \\ Asimina Kiourti
}


Copyrighted by

Jeffrey Scott Kula

2019 


\begin{abstract}
This dissertation introduces a new class of reconfigurable frequency selective structures (FSSs) to operate across a wide band of frequencies. Specifically, we explore various thick FSS designs with desirable properties, including sharp roll-offs for narrowband rejections and performance stability with incidence angle. By comparison, previous research has mostly focused on thin FSS structures, providing for limited degrees of freedom in designing the band rejection or band-pass properties of these filters. Similar to multi-stage filters, the attained higher-order selectivity enables frequency and bandwidth control. However, practical application also requires the ability to tune these structures.

The proposed designs introduce novel geometries to achieve reconfigurable frequency control for FSSs within the 6-14 GHz band. Various element designs of different physical configurations are explored, aimed at greater selectivity as well as dynamic tuning and control of the band rejection width. In addition to demonstrating each of these performance enhancements, we discuss and analyze the impact of combining them into a single design that incorporates the required tradeoffs to achieve desired design goals. As a way to provide design flexibility, a reconfigurable FSS based on an elliptical element is proposed and studied, taking advantage of its symmetry and
\end{abstract}


rounded edges. Specific band rejection designs are fabricated and tested to verify the design and simulations. Similar FSS structures can also be adapted to band-pass configurations. Given the validation and potential applications, we present a new path forward for these reconfigurable thick FSS designs. 


\section{Dedication}

To my mother, brother, and wife. 


\section{Acknowledgments}

Work of this magnitude can only be accomplished with the help and support of many individuals. First, I would like to acknowledge my advisor, Prof. John Volakis. In addition to his technical and research guidance, he has served as a mentor for me through regular conversations on both personal and professional development. I am grateful for his endless patience in helping me see this project through to its end, after my long path to graduation. I would also like to thank and acknowledge Prof. Niru Nahar for agreeing to serve as my co-advisor. Her knowledge and expertise has been instrumental in enabling me to take these last steps across the finish line. I would additionally like to acknowledge Prof. Asimina Kiourti, who not only served on my dissertation committee, but also played an important role in my research progress during its early stages.

I would also like to thank many others from the ElectroScience Laboratory who played key roles in my progress and development. I want to express my gratitude to Prof. Prabhakar Pathak, who served a critical role in my development of this research, providing me with a foundational understanding of both theory and applied research. I also want to thank Prof. Kubilay Sertel, who assisted me on numerous occasions on this and other research projects and helped me develop an appreciation for research practices. I also want to acknowledge Prof. Chi-Chih Chen, Prof. Fernando Teixeira, Prof. Bob 
Burkholder, Prof. Walter Burnside, Prof. Robert Garbacz, Prof. Edward Newman, and Prof. Rob Lee, all of whom contributed in important ways to my research and academic success during my time at Ohio State, as well as the staff at the ElectroScience Laboratory who provided constant assistance and support.

I want to acknowledge and express my appreciation for several folks who additionally contributed to my research. As this project was supported by the Dayton Area Graduate Studies Institute (now Defense Associated Graduate Student Innovators as part of the Southwestern Ohio Council for Higher Education), I'd like to thank Dr. Elizabeth Downie, Ms. Kim Elam, and others from DAGSI and SOCHE who helped make this possible. I'd also like to thank the Air Force Research Laboratory for its support, resources, and expertise, especially Mr. Mark Minges and Dr. Dan Janning. Finally, I'd like to thank Dr. Felix Miranda from NASA Glenn Research Center for his support of this work.

And of course, none of this would have been possible without the endless love and support from my family. In addition to both their explicit and implicit support, their collective work ethic has also served as a great example. I thank my mother, Cindy, for her ongoing belief and encouragement to persevere, as well as the patience to allow me to ultimately finish after a long and winding journey. I thank my brother, Kevin, for his support and for providing me with the confidence to believe that I can achieve anything I set my mind to. And I thank my wife, Nancy, whose dedication to her own research combined with her constant reassurance has helped inspire me to finally finish. 


\section{Vita}

December 28, 1978

June 2002

June 2005
..Born - Parma, OH USA

B.S. Electrical and Computer Engineering The Ohio State University - Columbus, $\mathrm{OH}$

M.S. Electrical and Computer Engineering The Ohio State University - Columbus, OH

\section{Publications}

J. S. Kula, N. K. Nahar, and J. L. Volakis, "Reconfigurable band rejection frequency selective structures," IEEE Transactions on Antennas and Propagation, submitted 2018.

J. S. Kula, N. K. Nahar, and J. L. Volakis, "Reconfigurable band rejection and band-pass frequency selective structures," USNC-URSI National Radio Science Mtg., July 2018.

J. S. Kula and J. L. Volakis, "Reconfigurable band rejection and band-pass frequency selective structures," USNC-URSI National Radio Science Mtg., July 2017.

J. S. Kula, P. H. Pathak, and J. L. Volakis, "Reconfigurable band rejection and band-pass frequency selective structures," IEEE International Symp. on Antennas and Propagation, pp. 890-891, July 2015.

J. S. Kula, P. H. Pathak, and J. L. Volakis, "Reconfigurable band rejection frequency selective structures," USNC-URSI National Radio Science Mtg., p. 149, July 2014.

J. S. Kula, D. Psychoudakis, W.-J. Liao, C.-C. Chen, J. L. Volakis, and J. W. Halloran, "Patch-antenna miniaturization using recently available ceramic substrates," IEEE Antennas and Propagation Magazine, vol. 48, no. 6, pp. 13-20, Dec. 2006. 
J. Kula, D. Psychoudakis, C.-C. Chen, J. L. Volakis, and J. H. Halloran, "Patch antenna miniaturization using thick truncated textured ceramic substrates," IEEE Antennas and Propagation Society Symp., pp. 3800-3803, June 2004.

\section{Fields of Study}

Major Field: Electrical and Computer Engineering

\section{Specialization:}

Electromagnetics

Communications 


\section{Table of Contents}

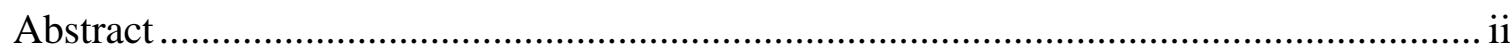

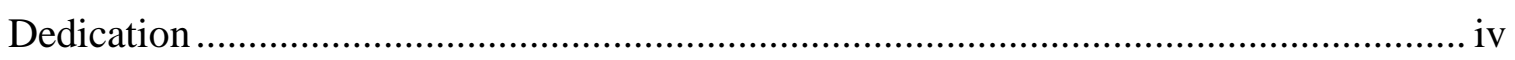

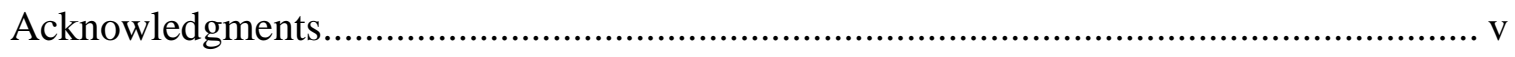

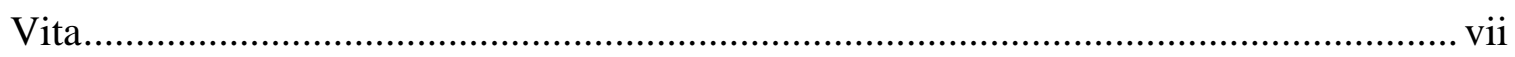

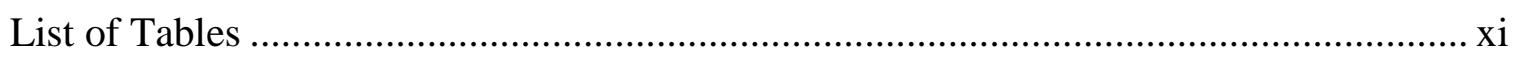

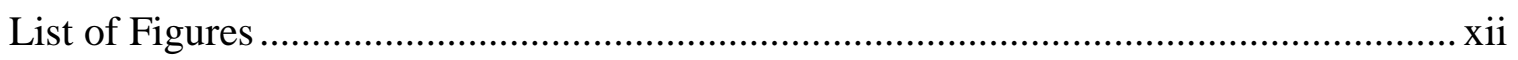

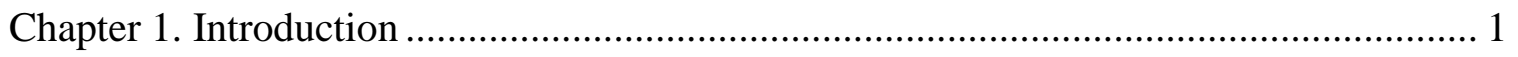

Chapter 2. Overview of Thick Frequency Selective Structures and Reconfigurability...... 9

2.1 Fundamental Design and Operating Principles .......................................... 9

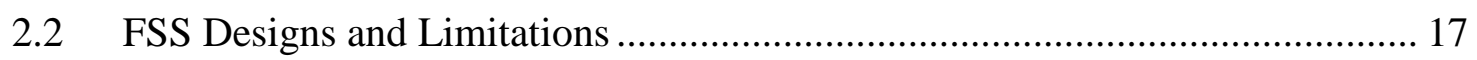

2.3 Proposed Designs and Key Contributions.................................................... 22

Chapter 3. Frequency Selectivity and Bandwidth Control ........................................... 26

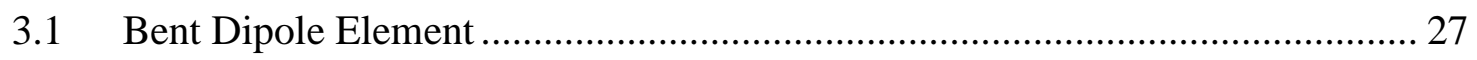

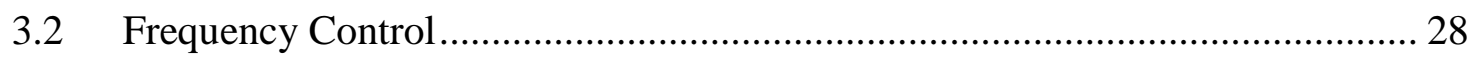

3.3 Highly Selective Rejections .................................................................. 31

3.4 Bandwidth Control .................................................................................... 33

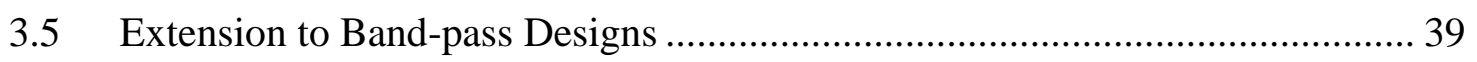

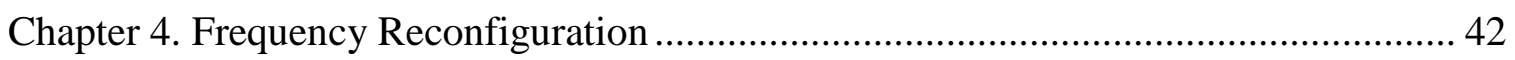

4.1 Reconfigurable Multi-Arm Bent Dipole ....................................................... 43

4.2 Reconfigurable Angled Dipole...................................................................... 49

4.3 Multi-band Designs .................................................................................. 51

Chapter 5. Performance Stability with Incidence Angle ............................................ 57

5.1 Bent and Angled Dipole Designs .............................................................. 58

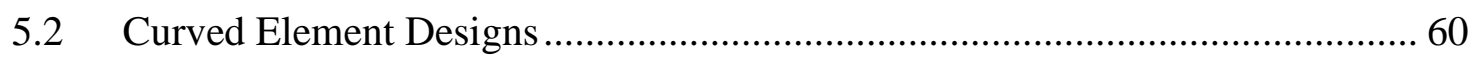

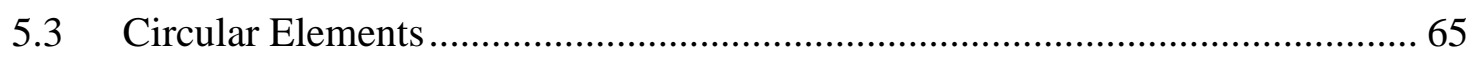




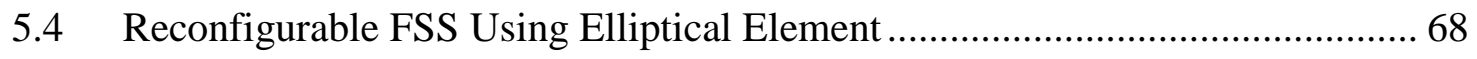

Chapter 6. Fabrication and Measurements for Validation ........................................... 74

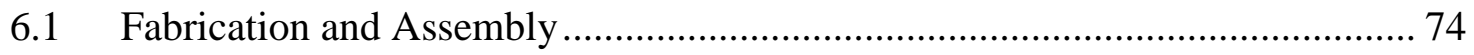

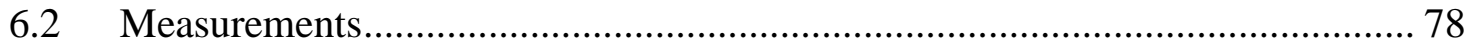

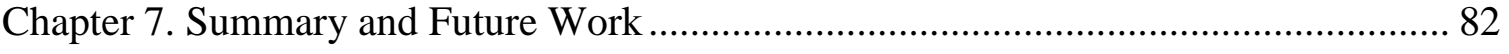

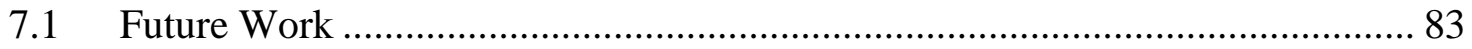

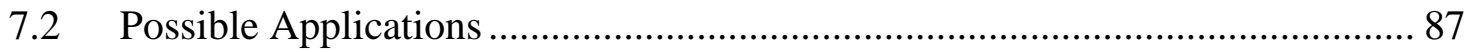

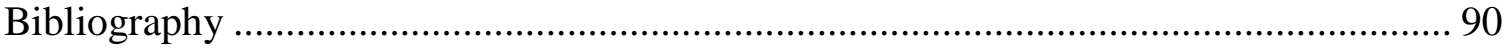




\section{List of Tables}

Table 1. Impact of varying dipole width on relative bandwidth................................. 36

Table 2. Impact of varying dielectric substrate thickness on relative bandwidth............ 38

Table 3. Rejection frequency and relative bandwidth for angled dipole FSS design...... 51 


\section{List of Figures}

Figure 1. Example planar frequency selective surface design. ................................... 3

Figure 2. Example thick frequency selective structure design. .................................... 5

Figure 3. Unit cell for thick FSS using simple dipole element geometry...................... 10

Figure 4. Creation of thick FSS, from unit cell to 2-D periodic array........................... 11

Figure 5. Thick frequency selective structure produced using simple dipole element..... 12

Figure 6. Reduction of infinite periodic array to single unit cell, with periodic

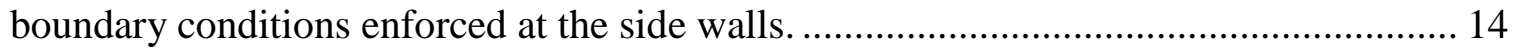

Figure 7. Foundational unit cell for proposed thick FSS designs, with incidence angle.. 23

Figure 8. Bent dipole geometry and unit cell....................................................... 27

Figure 9. Frequency shift in $S_{21}$ response by varying $L_{f u l l}$ values for bent dipole. .......... 29

Figure 10. Frequency shift in $\mathrm{S}_{21}$ response by varying $L$ values for bent dipole............. 30

Figure 11. Transmission response demonstrating improved selectivity for bent dipole

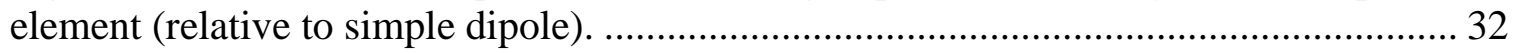

Figure 12. PEC element width for bent dipole design. .......................................... 34

Figure 13. Performance for varying PEC element widths: (a) $S_{11}$ and (b) $S_{21} \ldots \ldots \ldots \ldots . . . . .35$

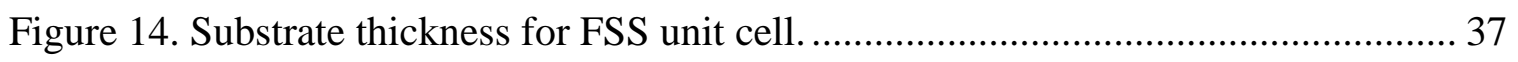

Figure 15. $\mathrm{S}_{21}$ performance for varying dielectric substrate thickness. ......................... 38

Figure 16. Slot design for band-pass response...................................................... 39 
Figure 17. $\mathrm{S}_{21}$ performance for slot design producing band-pass frequency response..... 40

Figure 18. Multi-arm bent dipole element for reconfigurable frequency control............ 44

Figure 19. Multi-arm bent dipole unit cell and FSS array....................................... 45

Figure 20. Ramped multi-arm bent dipole element. ............................................... 46

Figure 21. Frequency control and reconfiguration across 6-12 GHz using ramped

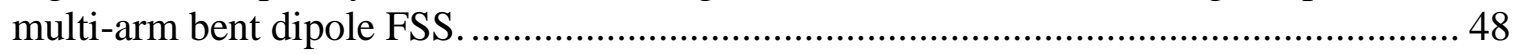

Figure 22. Higher-order resonances for ramped multi-arm dipole FSS. ...................... 49

Figure 23. Thick FSS unit cell for reconfigurable angled dipole. ............................... 50

Figure 24. Multiple rejections produced by interleaving two simple dipole designs...... 52

Figure 25. Multi-band unit cell design using interleaved multi-arm bent dipole

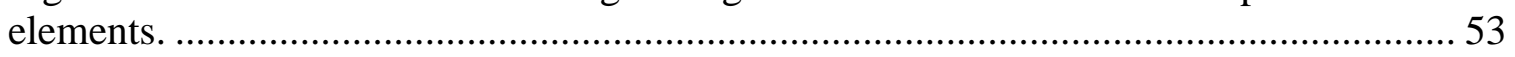

Figure 26. Dual-band rejection response for interleaved multi-arm bent dipoles. ........... 54

Figure 27. Impact of varying gap size on rejection frequency. ................................. 55

Figure 28. $(\theta, \phi)$ orientations for incidence angle used in FSS design. ........................... 58

Figure 29. $S_{21}$ performance for angled dipole by varying incidence angle $(\theta)$, for

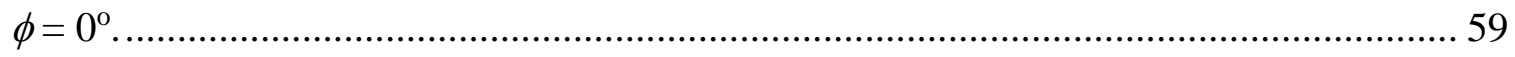

Figure 30. Example dipole elements with additional bends. ..................................... 60

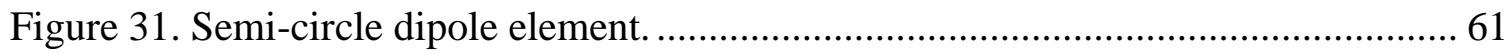

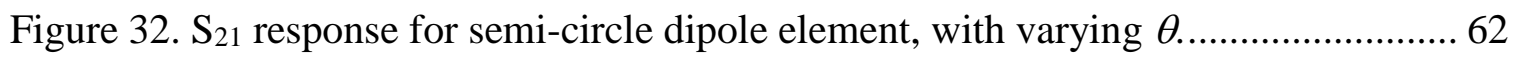

Figure 33. Side view of semi-circle dipoles: (a) original element and (b) torus element. 63

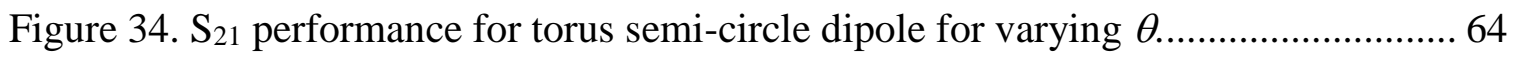

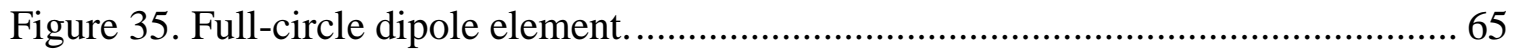

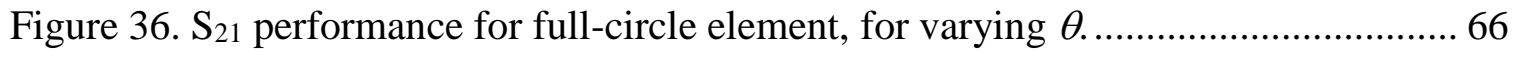


Figure 37. Full-circle dipole element, with gaps for reconfiguration.

Figure 38. $\mathrm{S}_{21}$ performance for circular element with gaps, for varying $\theta$.

Figure 39. Elliptical element design: (a) 3-D unit cell, (b) full ellipse, (c) ellipse with gaps at top and bottom of the element, and (d) ellipse with two symmetric gaps near the top of the element.

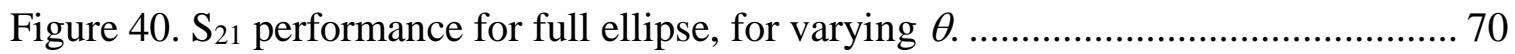

Figure $41 . \mathrm{S}_{21}$ performance for ellipse with gaps at top and bottom, for varying $\theta . \ldots \ldots . .71$

Figure 42. $\mathrm{S}_{21}$ performance for ellipse with two symmetric gaps near top, for varying $\theta .72$

Figure 43. $S_{21}$ performance for different reconfigurations of elliptical FSS element, with normal incidence angle $\left(\theta=0^{\circ}, \phi=0^{\circ}\right)$.

Figure 44. Creation of strip layers for fabricated designs: (a) side view of unit cell, (b) front view of unit cell with dimensions, and (c) 1-D strip created from unit cell....... 76

Figure 45. Fabricated and assembled FSS array: (a) full 2-D array and (b) unit cell...... 77

Figure 46. (a) Measurement set-up and (b) zoomed-in photo of FSS array/antenna configuration.

Figure 47. Measurement results for two fabricated FSS arrays, compared with simulations. 80

Figure 48. Example RF MEMS switch. 84

Figure 49. Circular-based element for reconfiguration 84

Figure 50. $\mathrm{S}_{21}$ performance for reconfigurable circular element for varying $\theta$. 86 


\section{Chapter 1. Introduction}

Communications, sensing, imaging, and navigation functions are integral to military applications such as the Air Force Research Laboratory's (AFRL) cognitive

layered sensing $[1,2]$. Central to these functions are radio frequency (RF) front-ends that are frequency agile, wideband, reconfigurable, very small in size and weight, and of low power - a necessity for future designs. Such RF front-ends can be used for cognitive sensing enabling remote reconfiguration and for terrestrial and airborne communications [3, 4]. Further, these functions need to be achieved in a congested electromagnetic (EM) environment, all while satisfying the increasing demands for multifunctionality. As the number of wireless systems, bandwidth, and wireless data volume increase, communication becomes more prone to interference. Therefore, a wideband system with interferer rejection capabilities is a necessity in future wideband antenna and front-end design.

Frequency reconfiguration for ultra-wideband (UWB) systems can be accomplished in several ways. Specifically, we can choose to (1) reconfigure the geometry of the antenna aperture using switches to control the inter-element capacitance, (2) integrate filters or geometrically reconfigure the balanced feed, and/or (3) introduce FSSs or reconfigure the ground plane's location or impedance. Indeed, the possibilities 
for array bandwidth and impedance reconfiguration are rather extensive. These approaches provide an incredible number of choices for bandwidth reconfiguration or signal rejection.

An attractive approach to achieve these designs is to employ a frequency selective surface [5-8]. These planar surfaces are created from two-dimensional (2-D) periodic repetitions of a unit cell containing a metallic (or sometimes dielectric) patch or aperture element, as pictured in Figure 1. Due to their design, these surfaces can exhibit a selective effect on the frequency, polarization, and incident angle of electromagnetic waves. In this way, these surfaces act as spatial filters and serve to reflect, transmit, or absorb electromagnetic fields based on the frequency of the field, enabling for rejection bands and/or passbands within a desired frequency range. 


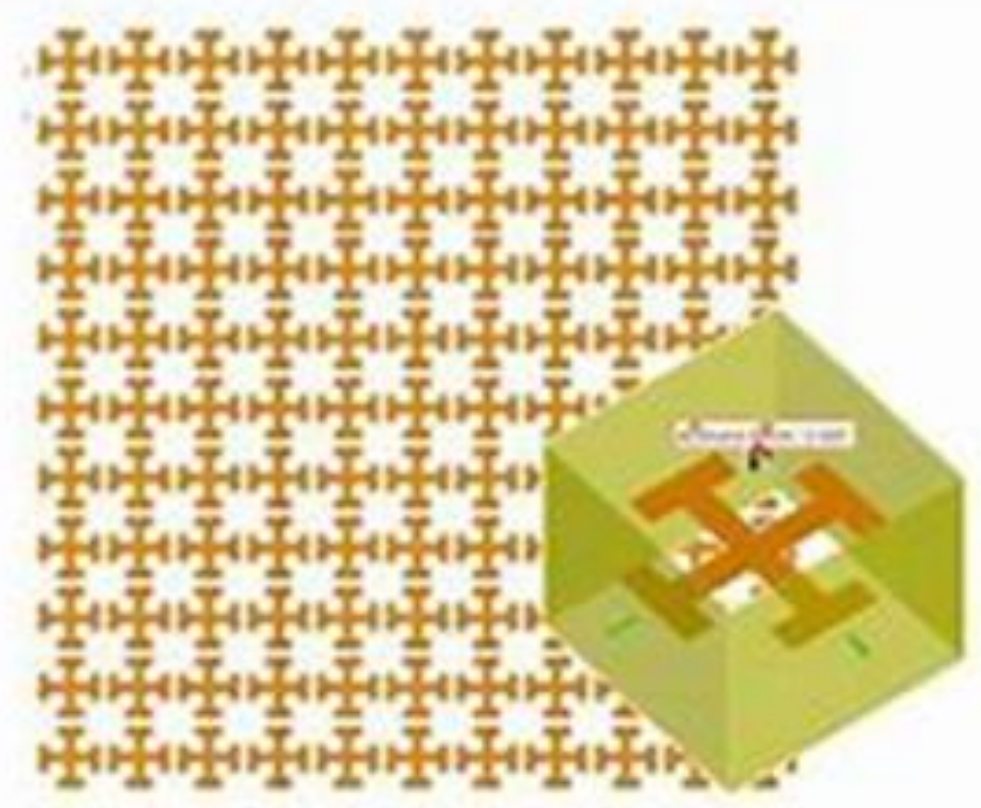

Figure 1. Example planar frequency selective surface design.

Typical frequency selective surface designs utilize periodic arrays of conducting elements (as shown in Figure 1) or slots in conducting screens, and these arrangements enable a resonant interaction of waves with the conductor segments. These elements can take a variety of shapes, and the foundational shapes are categorized into groups based on the types of filter responses that they produce (e.g., band-stop, band-pass, low-pass, and high-pass) [9]. For narrowband and/or multi-band applications, the band-stop and bandpass designs are of most interest. For a frequency selective surface design, these elements are often printed on a dielectric substrate layer for structural support, which can also impact the frequency response. In all, the element geometry, substrate, superstrate, element conductivity, and inter-element spacing are all design parameters that can play 
an important role in the performance of a frequency selective surface. In this way, frequency selective surfaces have been employed in a wide range of applications. These designs serve as filters for many systems, such as antenna radomes [10, 11], RCS reduction of an antenna array $[12,13]$, transmission or reflection of EM waves, reflectors [14, 15], and modern metamaterials [16].

However, existing frequency selective surface designs are thin, and as a result do not have sufficient flexibility to achieve reconfiguration across a large band. Because traditional frequency selective surface designs employ planar arrangements, they typically exhibit poor filtering responses similar to single-stage filters. Therefore, these surfaces cannot produce sharp stop bands, which are highly desirable to enable the supported antenna (e.g., communication system) to continue its normal operation. These planar surface designs also have responses that vary with incidence angle - all unavoidable consequences of the way in which they are constructed $[5,6]$.

In short, thin frequency selective surface designs are narrowband and are insufficient to serve military and commercial wireless needs due to the large spectrum utilization in current RF systems. Notably, while past operational frequencies did not often exceed X-band and transceivers were mostly narrowband, future systems will regularly require operation up to $30 \mathrm{GHz}$ [17]. As a result, transceivers will need to operate across large bandwidths. In those situations, it will be required to employ aperture filters that transmit/receive within small frequency windows across the entire available bandwidth. This is essential to ensure large signal-to-noise ratios. We 
therefore look to solutions that exhibit broadband performance with high frequency selectivity.

In order to provide greater selectivity, a class of thick frequency selective structures (FSSs) was considered [18, 19] to provide design flexibility and frequency control. In contrast to planar surface designs, a thick FSS creates a 2-D periodicity perpendicular to the plane of the element as displayed in Figure 2, and in doing so introduces higher-order filtering. However, this class of arrays lacks adequate tuning capability for practical application. Additionally, these thick FSSs still leave room for even higher-order filtering through more selective designs.

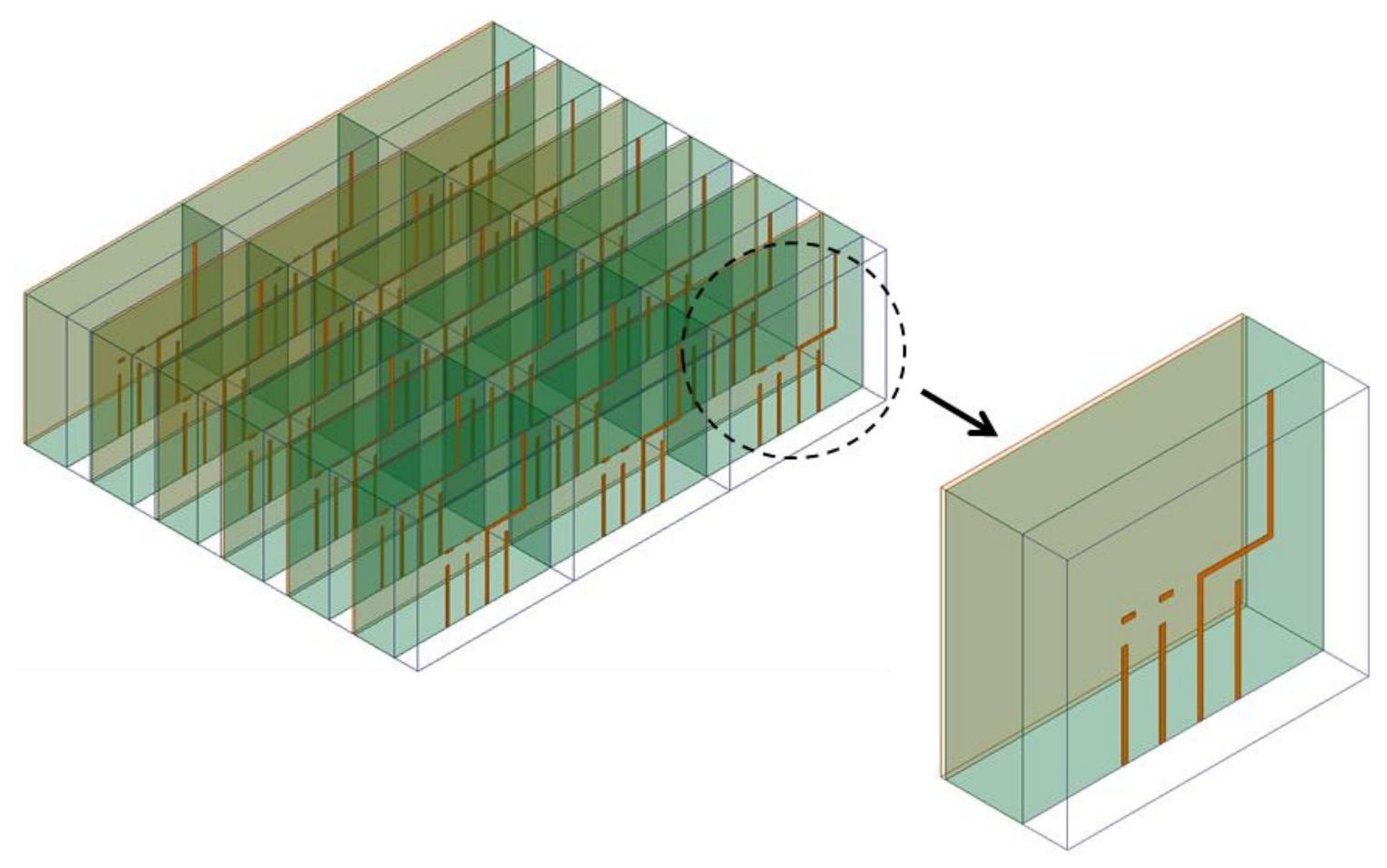

Figure 2. Example thick frequency selective structure design. 
The objective of this research is to design, optimize, and analyze a novel RF reconfiguration technique for UWB systems. We expand upon the thick FSSs in [18] to demonstrate RF reconfiguration and agility by tuning a narrowband design for operation across a wide range of frequencies. We introduce a new technique to control the frequency response of a multi-band notch filter by dynamically adjusting the FSS geometry of the thick unit cell. Previous designs demonstrated multi-band rejections for planar surfaces, but did not enable tuning/control of the frequency band via geometry reconfiguration.

The novelty of this dissertation is the exploration of thick FSS designs, and the extension to RF reconfigurability that has yet to be exploited for this class of arrays. We not only develop this new technique, but also extend it to thick structures. Such thick FSSs have many more degrees of freedom and can therefore allow for much improved band selectivity and lower profile with respect to current designs. We examine the viability of such tunability, stop-band sharpness, and verification via full-wave simulations and measurements.

Several methods are proposed for RF reconfiguration suitable for wideband, multi-function applications, including a novel class of thick FSSs with desirable properties such as sharp roll-offs and narrow rejections. Similar to multi-stage filters, this higher-order selectivity enables frequency and bandwidth control. The proposed designs allow for reconfigurable RF tuning and control of the rejection bandwidth. Different geometries are explored in terms of frequency and bandwidth control, and the concept extends to both band rejection and band-pass designs. We then use these 
geometries to analyze designs for stability with respect to incidence angle - incorporating multi-band operation and frequency control, as well as optimization for a more compact and efficient design.

These multi-function designs are ultimately optimized for efficiency and cost, with a big-picture focus on system resource management for practical implementation. The designs also incorporate an understanding of the integration necessary for practical application at a system level, as the resulting FSS layers must be fully integrated with antennas and all of the other back-end components for successful operation in the field. These frequency-agile, narrowband designs will be useful for future military and/or communication applications, where there is an ongoing drive for multi-function operation within a congested EM spectrum [20,21]. An application of interest is to employ proposed band rejection filters as reconfigurable radomes over broadband antennas, providing diverse and significant operation for future Air Force systems [22]. The proposed elements support highly improved selectivity and lower profile for RF reconfiguration, and as such, they can enable dynamic adaptation to a changing environment.

Moving forward, we expect that thick FSSs will provide the very much needed RF agility for filtering desired signals across large bandwidths. This dissertation represents foundational work for this new research direction, as thick FSS studies will likely follow the path of multilayered and reconfigurable printed and multilayer patch antennas [23-25], an area that has seen consistent growth since the mid-1990s. As such, 
these structures potentially represent the beginning of a large and broad field of study that will lead to new characterization methods. 


\section{Chapter 2. Overview of Thick Frequency Selective Structures and Reconfigurability}

Traditional frequency selective surfaces [5, 6] employ planar arrangements, and typically exhibit poor filtering responses similar to single-stage filters. For higher-order

filtering responses, recent literature has explored "thick" frequency selective structures (FSSs) to design rejection filters with sharp roll-offs. A class of thick FSSs was recently considered $[18,19]$ to add inductance and capacitance to the unit cell and provide design flexibility and frequency control for the FSS. In effect, these designs realize a higher-order multi-stage filter to achieve sharper roll-offs relative to the singlestage filtering exhibited in planar designs.

\subsection{Fundamental Design and Operating Principles}

An example thick FSS unit cell with a simple dipole element geometry is displayed in Figure 3. The unit cell is comprised of a printed element (typically metallic) sandwiched between an air layer and a dielectric substrate. As shown, the substrate separates the printed element from a PEC backing that completely covers the back side of the substrate layer. 


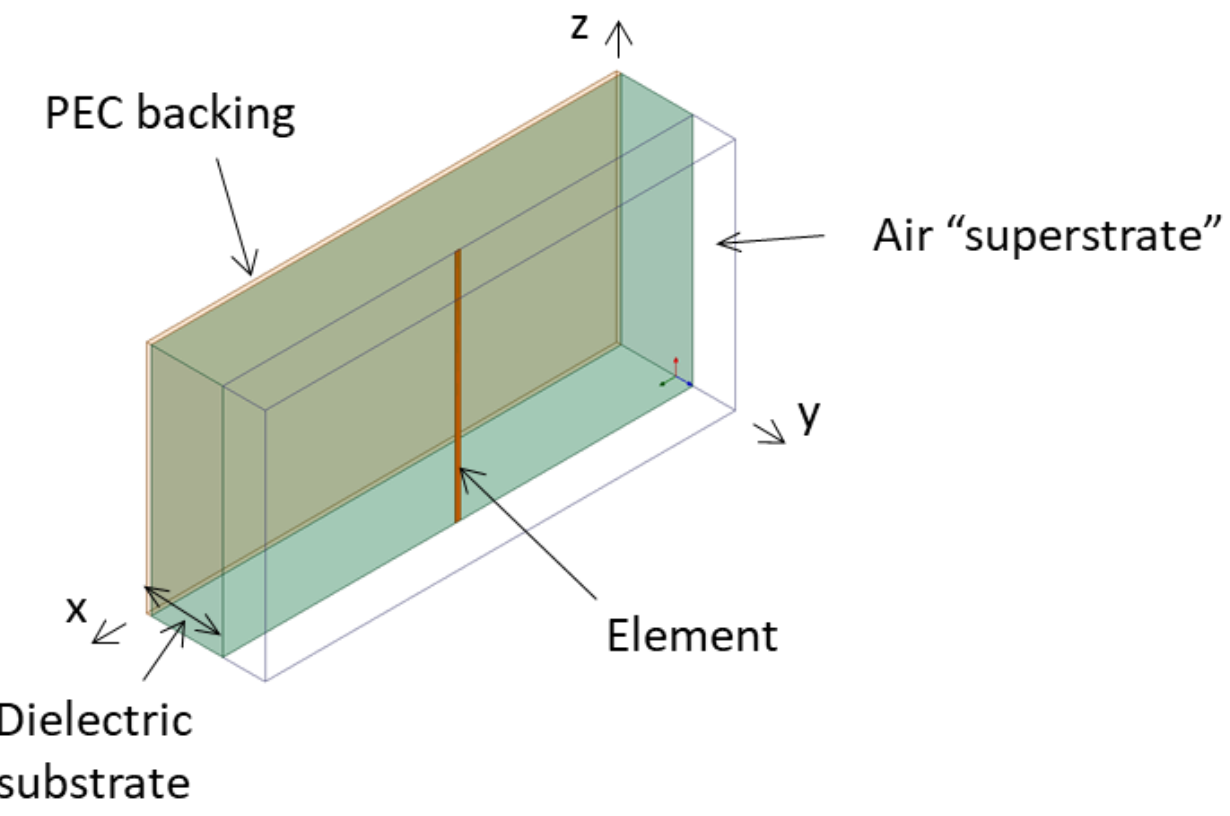

Figure 3. Unit cell for thick FSS using simple dipole element geometry.

In order to create the FSS array, the unit cell is replicated with a 2-D periodicity in the $\mathrm{x}$ - and $\mathrm{y}$-directions. The structure produced from this unit cell is depicted in Figure 4. As pictured, when the unit cell is replicated in the $\mathrm{x}$-direction, the individual cells join to form a 1-D array with solid air and substrate layers. Further, the PEC backing forms a solid ground plane for the strip. These strips are then stacked periodically in the y-direction to form a 2-D array. The resulting FSS is in fact a series of parallel PEC sheets (each in an xz-plane) acting as waveguides. Each row of printed elements is separated from its nearest PEC sheets by a dielectric substrate layer and an air "superstrate" layer, respectively. 


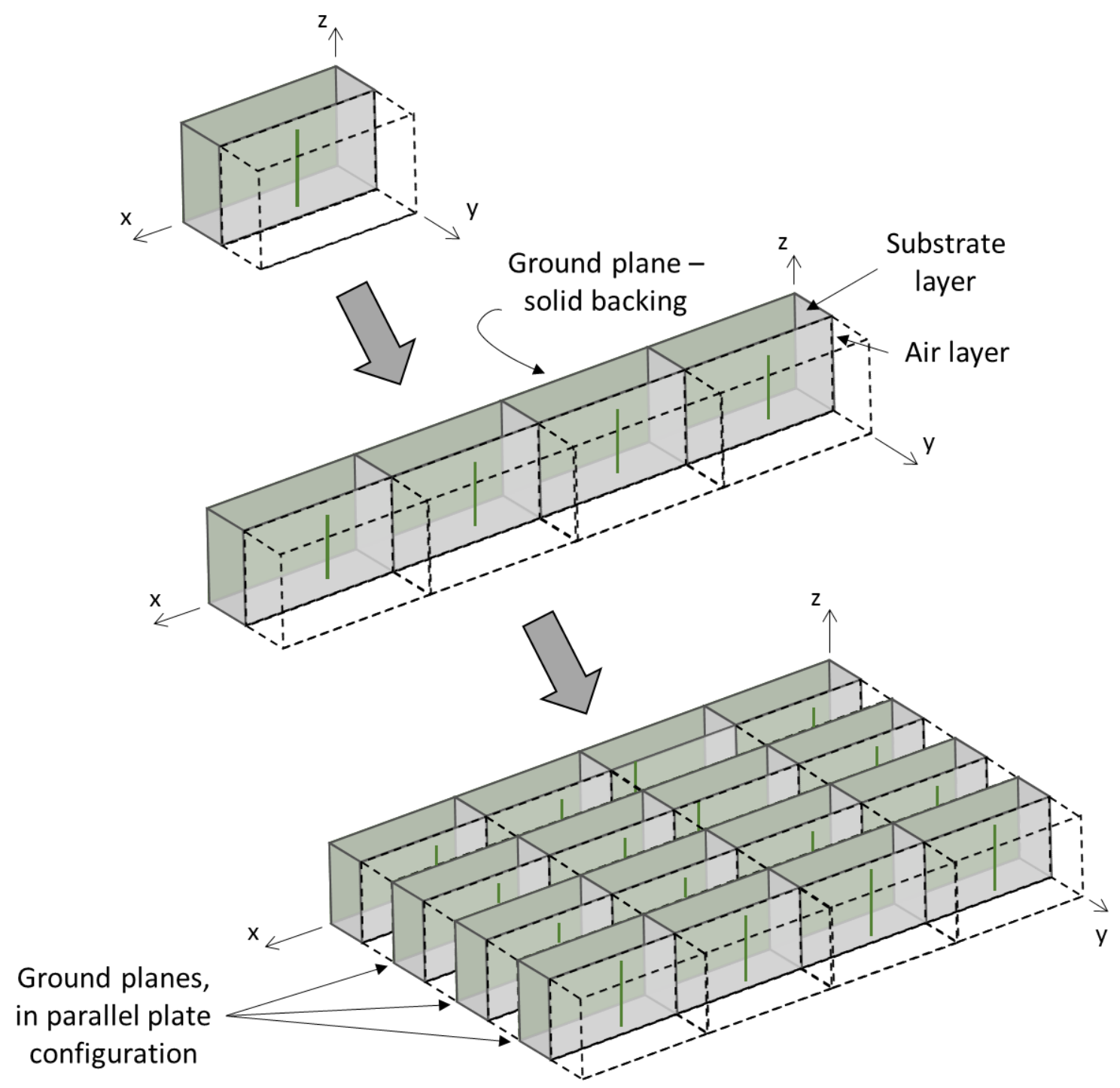

Figure 4. Creation of thick FSS, from unit cell to 2-D periodic array.

Figure 5 shows the complete thick frequency selective structure produced by the 2-D periodic arrangement of the unit cell from Figure 3. The normally-incident plane wave is $-z$-directed. So as previously noted, in contrast to the usual planar geometry, this thick FSS design utilizes elements with a thickness in the direction of propagation. 


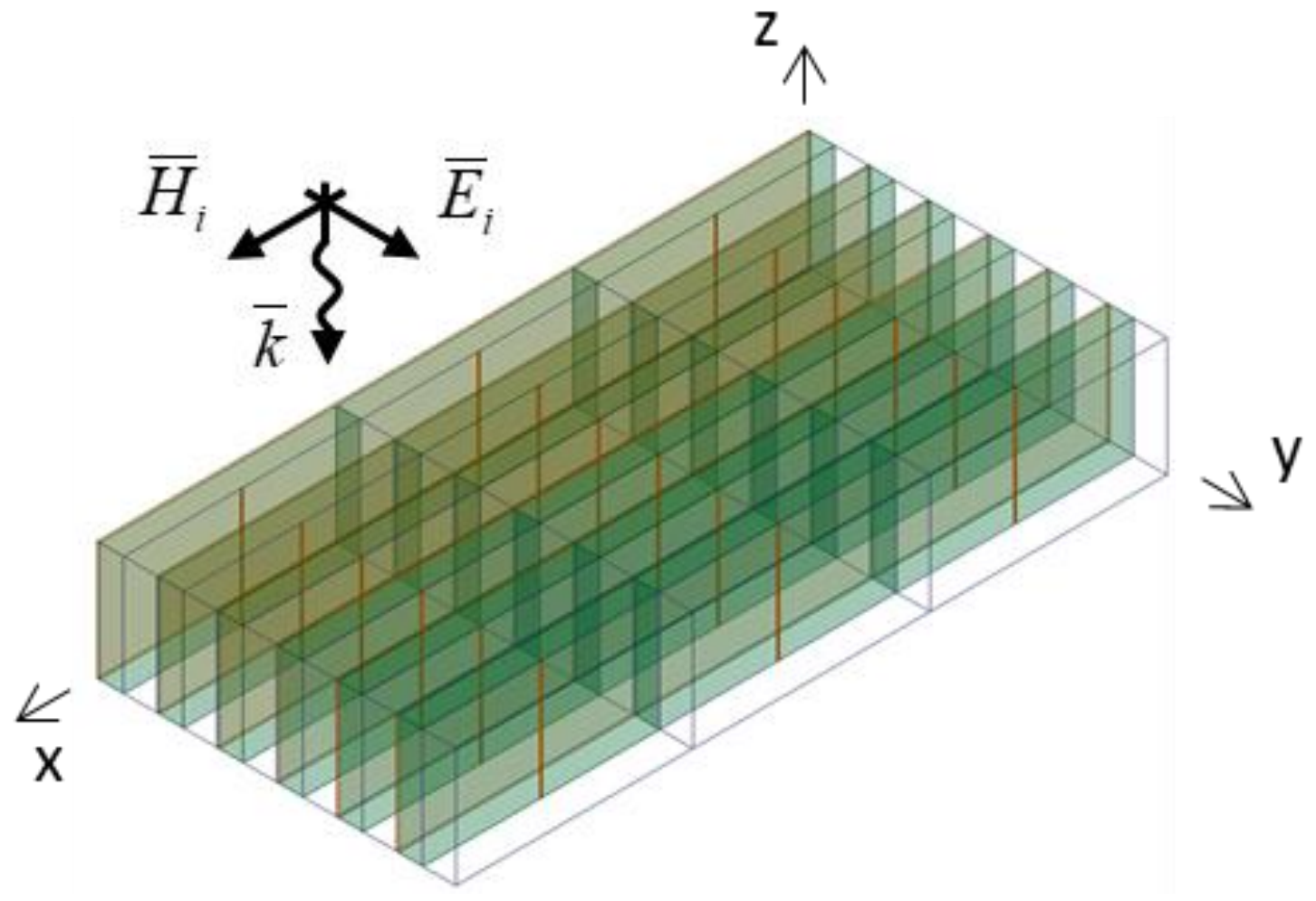

Figure 5. Thick frequency selective structure produced using simple dipole element.

The thick FSS operates for a linear electric field polarization that is received perpendicular to the printed strips, as pictured in Figure 5. Numerous methods have been developed to analyze frequency selective surfaces, and these approaches usually assume that the array is periodic and infinite in extent. For instance, one technique uses an equivalent circuit, where the various strip segments comprising an element are modeled as inductive and capacitive components on a transmission line. While the resulting circuit represents a straightforward way to calculate the reflection and transmission coefficients of an FSS screen, this method has accuracy issues past the resonant frequency and when modeling dielectric loading effects. 
Another method is a modal analysis, which has been the most successful in calculating the performance of an FSS. With this technique, an integral equation is derived to match fields scattered from the FSS to the current modes on the periodic surface. The fields are characterized by Floquet modes in space [26], while the current distribution induced in the conducting elements (by the incident field) is represented as a series of suitable basis functions. The integral equation is formed by applying standard boundary conditions at the conductors to match these two components. While the integral equation may be formulated using either a spatial or spectral domain approach, an efficient implementation can use various acceleration techniques to improve the convergence of the respective Floquet-mode series [27].

Various techniques may be utilized to solve this integral equation, including the method of moments $[28,29]$. Additionally, full-wave analysis can be performed by a hybrid finite element/boundary integral method [30], particularly useful in the analysis of multilayer periodic FSSs. Figure 6 demonstrates an example of the reduction of an infinite array to a unit cell. 


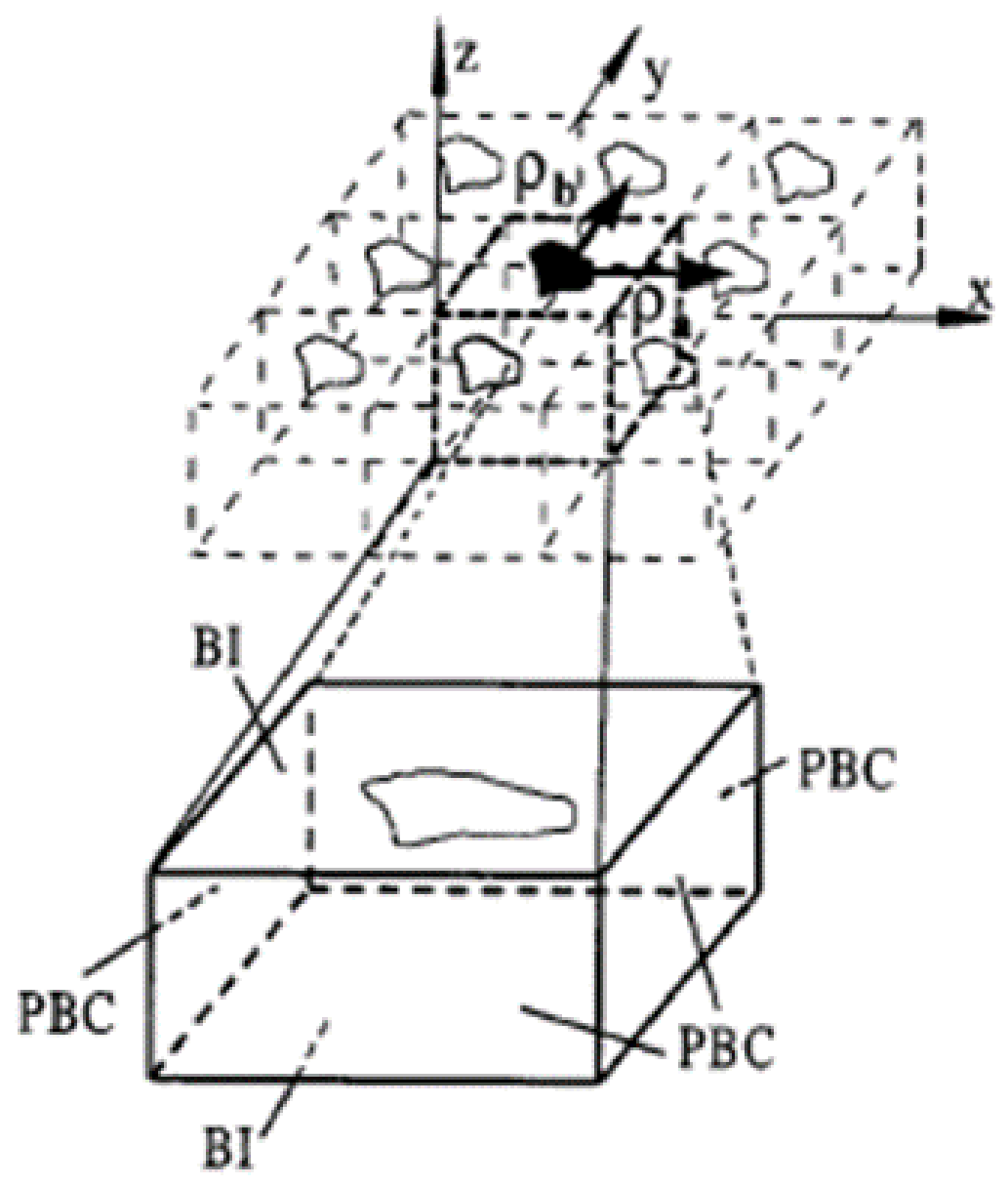

Figure 6. Reduction of infinite periodic array to single unit cell, with periodic boundary conditions enforced at the side walls.

As pictured, infinite periodic arrays are assumed to be periodic in the xy-plane, and the $(m, n)$ th cell of the array is obtained by shifting the $(0,0)$ th cell through the relation:

$$
\rho_{m n}=m \rho_{a}+n \rho_{b}
$$


Here, $\rho_{a}, \rho_{b}$ are the lattice vectors parallel to the $x y$-plane. For periodic excitation of the array with a linear phase factor, the fields in the array obey the periodicity conditions:

$$
\begin{aligned}
& \mathrm{E}\left(\mathrm{r}+m \rho_{a}+n \rho_{b}\right)=\mathrm{E}(\mathrm{r}) \boldsymbol{e}^{-j \mathrm{k}_{t 00} \cdot\left(m \rho_{a}+n \rho_{b}\right)} \\
& \mathrm{H}\left(\mathrm{r}+m \boldsymbol{\rho}_{a}+n \boldsymbol{\rho}_{b}\right)=\mathrm{H}(\mathbf{r}) \boldsymbol{e}^{-j \mathrm{k}_{t 00} \cdot\left(m \rho_{a}+n \rho_{b}\right)}
\end{aligned}
$$

where

$$
\mathbf{k}_{\boldsymbol{t} \mathbf{0 0}}=k_{x 00} \hat{\mathbf{x}}+k_{y 00} \hat{\mathbf{y}}=k \sin \theta_{0} \cos \phi_{0} \hat{\mathbf{x}}+k \sin \theta_{0} \sin \phi_{0} \hat{\mathbf{y}}
$$

Here, $\left(\theta_{0}, \phi_{0}\right)$ characterizes the propagation direction of an incident plane wave [31].

By applying these periodicity conditions to the boundary integral, the computational domain of an infinite periodic structure can be reduced to a single unit cell. As shown in Figure 6, this field periodicity condition is enforced at the vertical boundaries of the unit cell $[32,33]$, denoted as "PBC"s. These periodic (or phase) boundary conditions (PBCs) relate the fields on opposing walls of the unit cell through a phase shift derived from (2). For instance, if $c_{m}$ represents the unknown field on one of the vertical walls of the unit cell, the value of the field on the opposite side wall $\left(c_{n}\right)$ is given by:

$$
c_{n}=c_{m} e^{-j \mathbf{k}_{t 00} \cdot \Delta \mathrm{r}}
$$


where $\Delta \boldsymbol{r}\left(=\boldsymbol{\rho}_{a}\right.$ or $\left.\boldsymbol{\rho}_{b}\right)$ is the vector joining the two edges [34]. In addition to enforcing the periodic (phase) boundary conditions on the side walls of the unit cell, the appropriate periodic Green's function must also be used.

The designs presented in this dissertation were simulated with ANSYS HFSS (high-frequency structure simulator) [35], which uses these periodic boundary conditions (referred to as master-slave conditions in the software) to calculate a solution for a periodic array from a single unit cell design. These simulations are for an incident planewave excitation (as pictured in Figure 5). Using this linear polarization, when adjacent strips are excited in phase, a unit cell of this periodic FSS array consists of a microstrip element that supports two quasi-TEM modes. The resulting operating principle is similar to that of elliptic waveguide filters employing a dual-mode resonator [36, 37].

Highly selective frequency control is explored by using the FSS as a band rejection filter. The thick FSS achieves frequency rejection by forming two resonances within the structure. These frequencies refer to the air mode and substrate mode resonances. When the (z-directed) incident plane wave is introduced with its electric field perpendicular to the dipole elements, two separate propagation paths are formed to link the input and output ports (i.e. the top and bottom faces of the thick FSS array). One of these is an air path, while the other is a dielectric substrate path - and both of these paths act like resonators. At lower frequencies, signals primarily travel through the air path. But at higher frequencies, the signals propagate through both paths. When the signals propagate through both parallel paths, they do so with different phase shifts. 
Since the guided wavelength in the substrate is shorter than that of the air mode, the substrate mode resonator resonates first. When the phase difference between signals traveling along the parallel substrate and air paths is $180^{\circ}$ (i.e. they are completely out of phase with one another), the signals cancel to produce a transmission cancellation [38]. Each transmission cancellation represents a rejection frequency of the FSS, and the resulting frequency response can be controlled based on the specific design, geometry, and dimensions.

\subsection{FSS Designs and Limitations}

While the foundational thick FSS concept provides improved filtering with respect to planar frequency selective surface designs, there are still limitations to performance and frequency control. Many recent studies have attempted to improve upon various facets of these designs, by focusing on specific performance characteristics. A common thread among these designs is the existence of tradeoffs. Not surprisingly, designing with a specific characteristic or metric in mind has an adverse effect on other performance features; therefore, an end design must typically be tailored for a specific application by balancing these tradeoffs against one another for optimally desired performance.

Although traditional planar frequency selective surfaces benefit from low size, weight, and power (SWaP) and cost, these single-layered designs are known to suffer from poor selectivity and narrow operating frequency range. Various techniques have been explored to overcome these limitations and enhance this performance, most notably 
via multilayered designs [39-41]. This technique offers additional design flexibility by cascading multiple frequency selective surface layers and embedding them within a dielectric (or separating them by a dielectric spacer) to improve filtering and enhance outof-band performance. This broadband response in the desired frequency range is obtained due to the discontinuity provided by the layers, resulting in faster roll-offs and smooth in-band frequency response relative to single-layer designs [42].

However, these multilayered designs based on planar arrays still have several limitations. For instance, the introduction of the finite dielectric layer produces effects caused by the interaction of fields adjacent to the conductors with the slab surface. Trapped waves can be produced, reducing the resonance even at normal incidence angles [43]. The selectivity of these designs can certainly improve. Additionally, embedded layered designs with multiple periodic layers present a challenge in syncing the periodicity from each for conformal designs on curved surfaces. Finally, whether single-layered or multilayered, planar frequency selective surface designs are unable to realize all attractive features (e.g., broadband performance, fast roll-offs, stability with incidence angle) simultaneously.

Since multilayered planar designs already add to the structure's profile, exploring thicker designs and their related benefits proves worthwhile. To this end, the introduction of thick FSS designs has opened new doors in achieving increased filtering responses for microwave technology. By using elements of a three-dimensional (3-D) nature, electric currents flow with components at right angles to the surface, thereby creating a geometry with reduced insertion loss that is also less sensitive to incidence 
angle [44]. A variety of inter-structure additions have been explored for specific design characteristics, such as microstrip line boards, metal plate bits, and shorting via holes $[45,46]$. Many of these techniques produce multiple resonances, as any desired number of transmission zeros and/or poles can be created at fixed frequencies by introducing additional propagating paths. These designs guide wave propagation and improve spatial filtering [47]. But despite high-order filtering and sharp roll-offs, thick FSSs still lack adequate tuning for practical application. Given the increasing demands for multi-load apertures and frequency agility, this tuning capability is critical.

A major challenge is that of achieving RF reconfiguration without having to actually modify the antenna or its feeding system. Such modifications are costly and complex, and may not be possible when the radar or communication system is already installed or available in the market. Nevertheless, such reconfiguration is important for protecting existing communication systems from stray and unwanted signals, including noise and jamming signals. Of particular interest is to increase the signal-to-noise ratio, and therefore ensure communication under interference.

A key technique for reconfiguration is through the introduction of an FSS. While research into frequency reconfiguration for thick structures has been limited, a variety of techniques have been explored for reconfiguration or multi-band rejections for traditional planar FSS deigns [48-50]. Many of these designs explore the use of active elements, to expand on passive frequency selective surfaces that are inflexible in performance after manufacturing [51, 52]. One technique involves mechanical alteration of the grid orientation [53] or of the elements themselves [54], but in general electrically-controlled 
devices are preferred for their flexibility and reliability. These electrical techniques can be separated into two categories - switchable and tunable. Switchable designs are used to toggle the frequency response at the desired band between transmission and reflection, often used to switch characteristics between an absorber and a reflector [55]. Tunable structures, on the other hand, are used to shift the resonant frequency within a certain frequency band. These tunable methods are the techniques of interest when developing the types of reconfigurable FSS designs explored in this dissertation.

Several types of devices have been utilized to achieve these tunable planar designs. For instance, magnetically-tunable ferrite substrates and ferroelectric materials have been used to shift both rejection bands and passbands by applying a bias voltage to change the substrate's dielectric constant $[56,57]$. This can result in absorption/reflection switching, but controlling the conductivity of the surface layers in this way is quite complex. Another example often explored is liquid tuning, such as with liquid crystals [58, 59]. However, the frequency tuning range of these designs is comparatively limited. Additionally, devices such as PIN diodes [60, 61], Schottky diodes [62], and varactors $[63,64]$ have been used for dual modes of EM switching and polarization selection. One drawback of these devices is that most resulting designs are asymmetrical. Therefore stability is a challenge, and the designs typically lead to small tuning range. Finally, radio frequency microelectromechanical system (RF MEMS) switches have been used for dynamic reconfiguration of planar frequency selective surfaces and antennas $[65,66]$. However, as all of these designs are for planar surfaces, 
they again result in relatively poor selectivity and control of the rejection band. And further, none of these techniques allowed for tuning via geometry reconfiguration.

While all of these techniques present possible options for tunable planar filters, there has been relatively little research towards their adaptation to thick designs. Almost all current publications on thick FSS designs focus on fixed frequencies, and therefore have limited use for tunable applications. Further, those that explore reconfiguration have primarily done so for normally-incident plane waves. Several designs have explored the use of a 3-D FSS element, but these simply extend the element from a planar design to include a depth in the direction of propagation. There is no rotation of the unit cell as with the thick FSS designs, and as a result the rejection bands and passbands still exhibit little selectivity $[46,54]$.

Some work has been done with electrically-controlled devices for thick FSSs, including the use of varactor diodes for frequency tuning [67]. While this design achieves a stable response with solid tuning range and selectivity, the structure has a large profile and a complex fabrication and assembly. Several mechanically-controlled techniques have also been explored for reconfiguration of thick FSS designs, including mechanical compression of spring resonators [68], controlled bending of a flexible FSS sheet [69], and physical rotation of the elements [70]. This last example demonstrates good flexibility for broadband operation and reasonable selectivity. However, not only does the design have a very large profile, but there also remains a need to drive the rotation of the elements for reconfigurable operation. Further, all of these mechanical 
tuning approaches would be slower than electrically-controlled techniques, and they also exhibit relatively limited tuning range.

\subsection{Proposed Designs and Key Contributions}

Given these limitations in the current development of tunable thick FSS designs, this dissertation explores a method to reconfigure resonances for operation across a wide band of frequencies. With reconfiguration as a primary focus, we utilize relatively simple element geometries to achieve selectivity improvement while avoiding a complex and expensive fabrication for validation. We develop straightforward design principles that enable elements to be constructed from their foundational parameters, all while operating in a higher frequency band (i.e. 6-14 GHz) than other tunable thick FSS designs. And we incorporate the concept of electrical switching to physically alter the element geometry, combining the benefits of speed and reliability with a direct design approach.

In this work, we build upon the thick FSS concept using novel elements to demonstrate dynamic RF reconfiguration within a single multi-function narrowband filter design. We improve upon these sharp band stops using unique elements that introduce greater inductance and capacitance for higher-order filtering, thus designing a new class of reconfigurable narrowband FSSs that operate across a wide band of frequencies. To do so, we introduce a new reconfiguration technique to control the frequency response of a multi-band notch filter by dynamically adjusting the unit cell geometry making up the 
thick FSS. These unit cell designs utilize the foundational structure depicted in Figure 7. The thick FSS is then created from the unit cell as discussed in Figure 4.

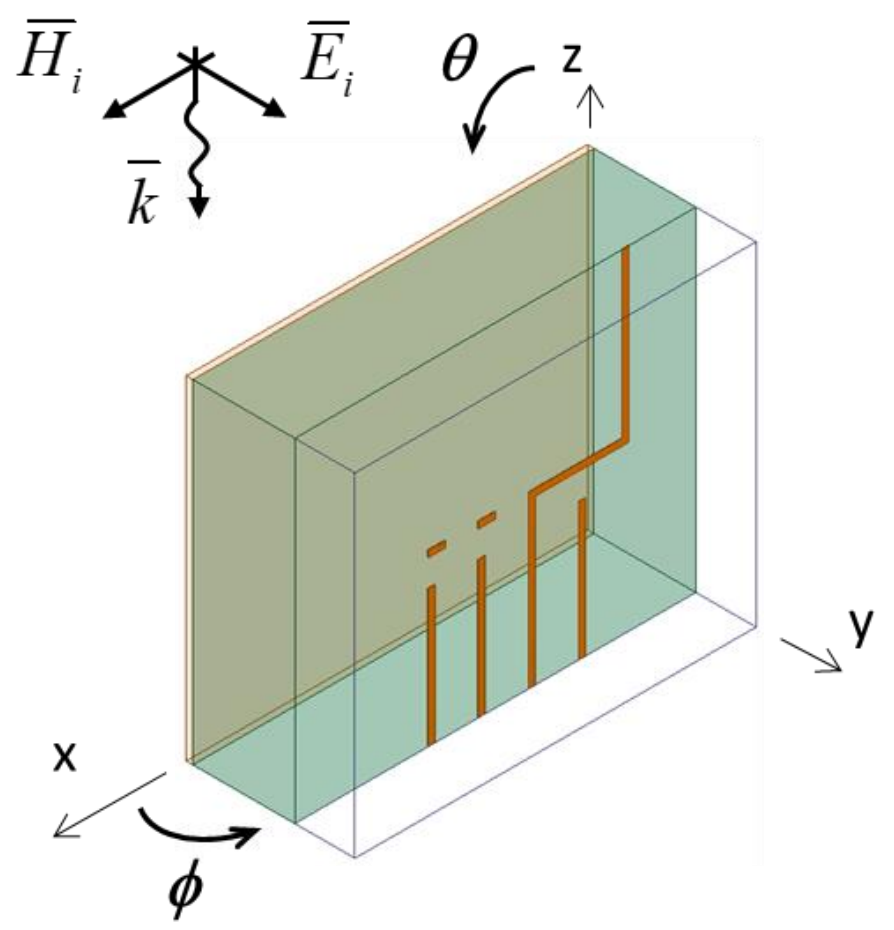

Figure 7. Foundational unit cell for proposed thick FSS designs, with incidence angle.

Also pictured is the coordinate frame for the parameters used to define the incidence angle of an incoming plane wave (i.e. $\theta$ and $\phi$ ) as used throughout the work. We reiterate that most of the analysis was accomplished for normal incidence (i.e. $\theta=0^{\circ}$, $\phi=0^{\circ}$ ), except for Chapter 5 which is specifically intended to analyze the impact of incidence angle on performance. Due to the extremely narrowband nature of these designs, even small frequency shifts due to incidence angle make stability a challenge for rejection bands or passbands - yet we successfully overcome this challenge by 
demonstrating a stable response across a wide range of incidence angles, as well as design considerations to control the bandwidth of the rejection frequencies.

The designed novel tunable structures demonstrate improved band selectivity and lower profile with respect to current designs. While the particular example highlighted in Figure 7 represents a reconfigurable multi-arm bent dipole extension of the simple dipole element, we explored many different FSS elements, geometries, and arrangements in analyzing the designs. The benefits, limitations, and tradeoffs for each will be discussed in the following chapters.

These improved designs have many potential applications, such as dynamically adjustable cover layers for multi-function systems [71] or frequency switching for communication/signal security [72]. The narrowband rejection designs can also be extended to exhibit band-pass capability, which enables total control of the operating frequency for one's own systems. While the exact architecture and test conditions may vary with applications, these concepts can be extended to critical technologies important to AFRL, such as simultaneous transmit/receive (STAR) capability [73], fieldprogrammable gate array analysis [74], and wideband digital beamforming [75].

This research provides key contributions to the area of tunable filters by exploring several improvements to the thick FSS design concept. Among these are (1) frequency selectivity with bandwidth control, (2) frequency reconfiguration for multi-band designs, and (3) performance stability with incidence angle. In the chapters that follow, we examine each of these significant design characteristics in succession, including the tradeoffs among them. We discuss the design evolution through a variety of thick FSS 
element geometries and unit cell parameters to develop sound design principles and provide meaningful analysis. This culminates in the cost-effective fabrication of two reconfigurable configurations of a singular, fundamental FSS element shape from commercial off-the-shelf materials - as well as the resulting measurements to validate our simulations. We begin by building upon the thick FSS concept to explore techniques to improve frequency selectivity and control for narrowband designs. 


\section{Chapter 3. Frequency Selectivity and Bandwidth Control}

Traditional planar frequency selective surfaces exhibit poor filtering responses similar to single-stage filters. "Thick" FSS designs improve upon this selectivity for both band rejections and passbands, by creating higher-order filtering responses with sharp roll-offs. By adding inductance and capacitance to the unit cell, these thick designs provide design flexibility and frequency control for the FSS, in effect realizing higherorder multi-stage filters with sharper roll-offs (versus single-stage filtering in planar designs).

However, even the thick FSS designs that have been studied to this point leave room for novel elements to increase this selectivity. While applications may vary considerably and dictate the exact type of element design desired, we pursued elements to generate highly selective rejection bands. These designs demonstrate benefits for narrowband applications. Further, while the primary focus is on rejections, the same principles can be extended to band-pass systems - such as in reconfigurable radomes over broadband antennas, designed to allow transmission for only a very narrow band of frequencies at a time. 


\subsection{Bent Dipole Element}

Through this research, we explored a variety of elements in an attempt to improve on the selectivity of thick FSS designs demonstrated in the literature [76]. In doing so, a straightforward reference design is the simple dipole used in [18]. Our technique explored ways to expand on this foundational element to introduce additional inductance and capacitance to the unit cell design. We found great success with a bent (zig-zag) dipole geometry as shown in Figure 8. This element geometry is primarily characterized by two parameters. First, $L_{f u l l}$ is used to represent the true physical length of the dipole, following the path along the contours of the element. Next, $L$ characterizes the z-directed "height" of the bent dipole, along the thickness of the FSS unit cell. Both of these parameters are depicted in Figure 8. In essence, the bent dipole design extends the length of the element without increasing the profile of the thick FSS.

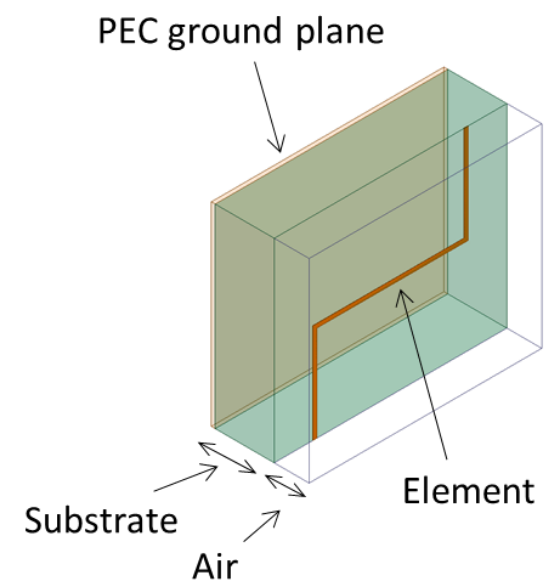

Figure 8. Bent dipole geometry and unit cell. 


\subsection{Frequency Control}

Frequency control is explored by using the FSS as a band rejection filter. Like a standard half-wavelength dipole, $L_{\text {full }}$ is initially selected to correspond to $\lambda_{g} / 2$, where $\lambda_{g}=\lambda_{0} / \sqrt{\left(\varepsilon_{r}+1\right) / 2}$ is the guided wavelength in the dielectric substrate (characterized by relative permittivity, $\varepsilon_{r}$ ). Note that since the energy is concentrated near the airdielectric interface, the effective permittivity is the average of the free space permittivity and the substrate permittivity. For a given set of selections for unit cell dimensions, substrate thickness, air layer thickness, and substrate permittivity, these two parameters $\left(L_{\text {full }}\right.$ and $\left.L\right)$ can be used to design for a specific rejection frequency.

Figure 9 demonstrates the impact that varying $L_{\text {full }}$ has on rejection frequency. For this case, $L$ is maintained at a constant value, so increasing $L_{\text {full }}$ results in a wider element shape. As is clear from the comparative $S_{21}$ plots, relatively small changes in $L_{f u l l}$ shift the rejection frequency somewhat significantly across a wide range of frequencies in the 8-12 GHz range. As expected, a longer element produces a lower resonant frequency. 


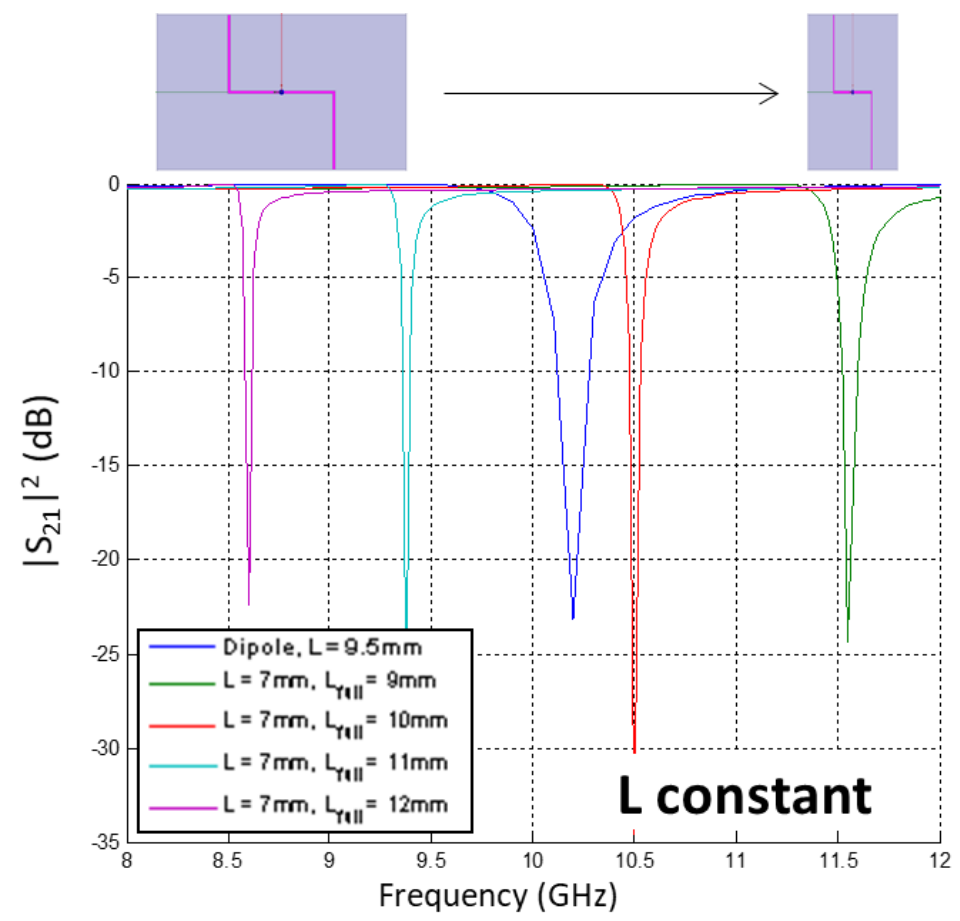

Figure 9. Frequency shift in $S_{21}$ response by varying $L_{f u l l}$ values for bent dipole.

Similarly, Figure 10 displays the impact that varying $L$ has on rejection frequency.

For this case $L_{f u l l}$ is fixed to a constant value, so increasing $L$ results in an element that is simultaneously taller and narrower. As we see from the $S_{21}$ plots, changing $L$ also shifts the rejection frequency. However, since the overall length is maintained constant, this frequency shift is much smaller. 


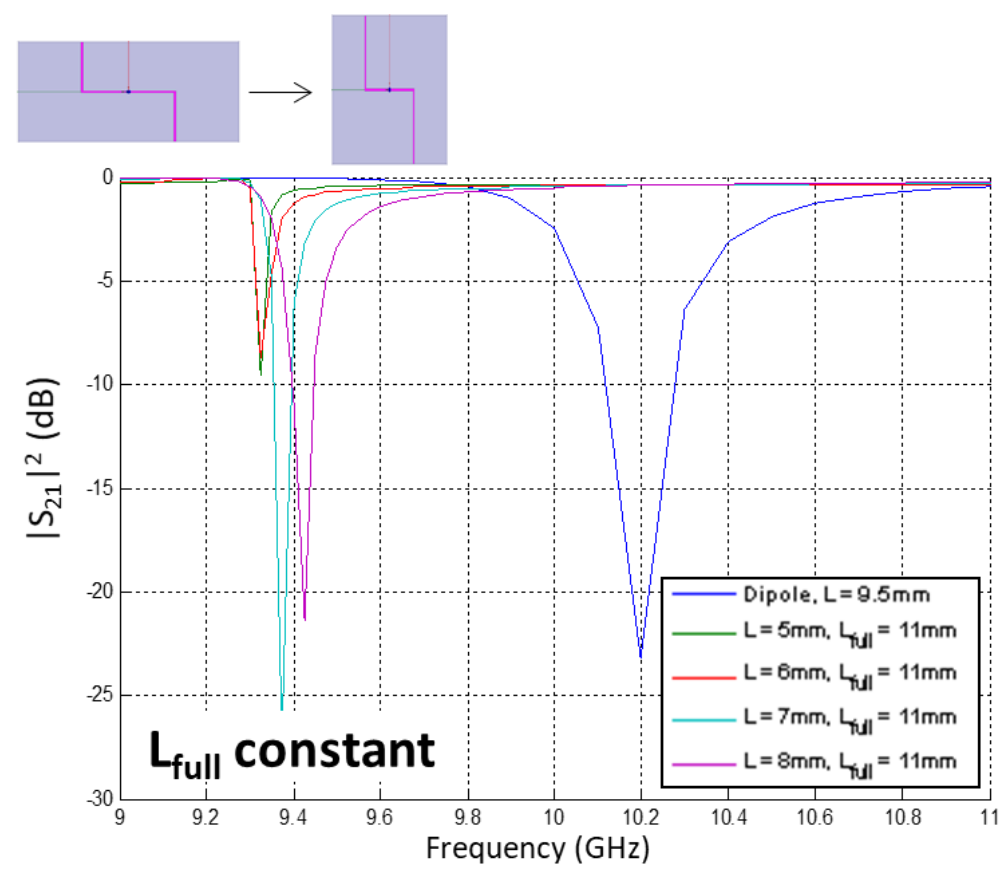

Figure 10. Frequency shift in $S_{21}$ response by varying $L$ values for bent dipole.

By carefully selecting these two parameters in combination with one another, an FSS designer has significant control over the specific frequency of operation. After $L_{f u l l}$ is selected for coarse tuning to control the frequency response, the $L$ parameter is used to fine-tune the frequency. The same element spacing was used between adjacent unit cell elements for all the FSS designs used in this parametric study, thus isolating the test parameter in each simulation and ensuring that coupling from adjacent elements did not play a role. And when varying $L$, the unit cell dimension varied in the z-direction, which would result in a profile change that must be considered when tuning the design frequency for practical applications. 


\title{
3.3 Highly Selective Rejections
}

Having achieved a design with readily adjustable parameters for precise frequency control, we next analyzed the increased selectivity obtained by the thick FSS design using the bent dipole element. While thick FSSs in general demonstrate improved selectivity relative to planar FSS designs, part of our objective is to propose and explore novel designs that enable highly selective rejections for applications and systems that require narrowband frequency operation. The bent dipole represents a successful design toward achieving this objective.

As an initial point of reference, we aimed to demonstrate the improved performance by comparing the selectivity of the bent dipole with that of the simple dipole in [18]. For the bent dipole element design, we maintained many of the same parameters in order to produce a direct comparison. Specifically, we used the following parameters to match the simple dipole design (as pictured in Figure 3):

\author{
Strip width $=0.1 \mathrm{~mm}$ \\ Unit cell width $(\mathrm{x}$-direction $)=10 \mathrm{~mm}$ \\ Air thickness $=1 \mathrm{~mm}$ \\ Substrate thickness $=1.524 \mathrm{~mm}$ \\ Substrate permittivity $=3.38$
}

To create the bent dipole, $L_{\text {full }}$ and $L$ were selected so as to match the rejection frequency of the $9.5 \mathrm{~mm}$ simple dipole. Using the design procedure as described above to 
control and fine-tune the frequency response, $L_{\text {full }}$ and $L$ were set to $10.2 \mathrm{~mm}$ and $6 \mathrm{~mm}$, respectively. Figure 11 shows a comparison of the transmission response for FSSs designed using these two elements. Both designs were simulated using ANSYS HFSS, and we replicated the simple dipole design using all the same design parameter values as in the reference paper. Both designs produce a rejection frequency at approximately $10 \mathrm{GHz}$.

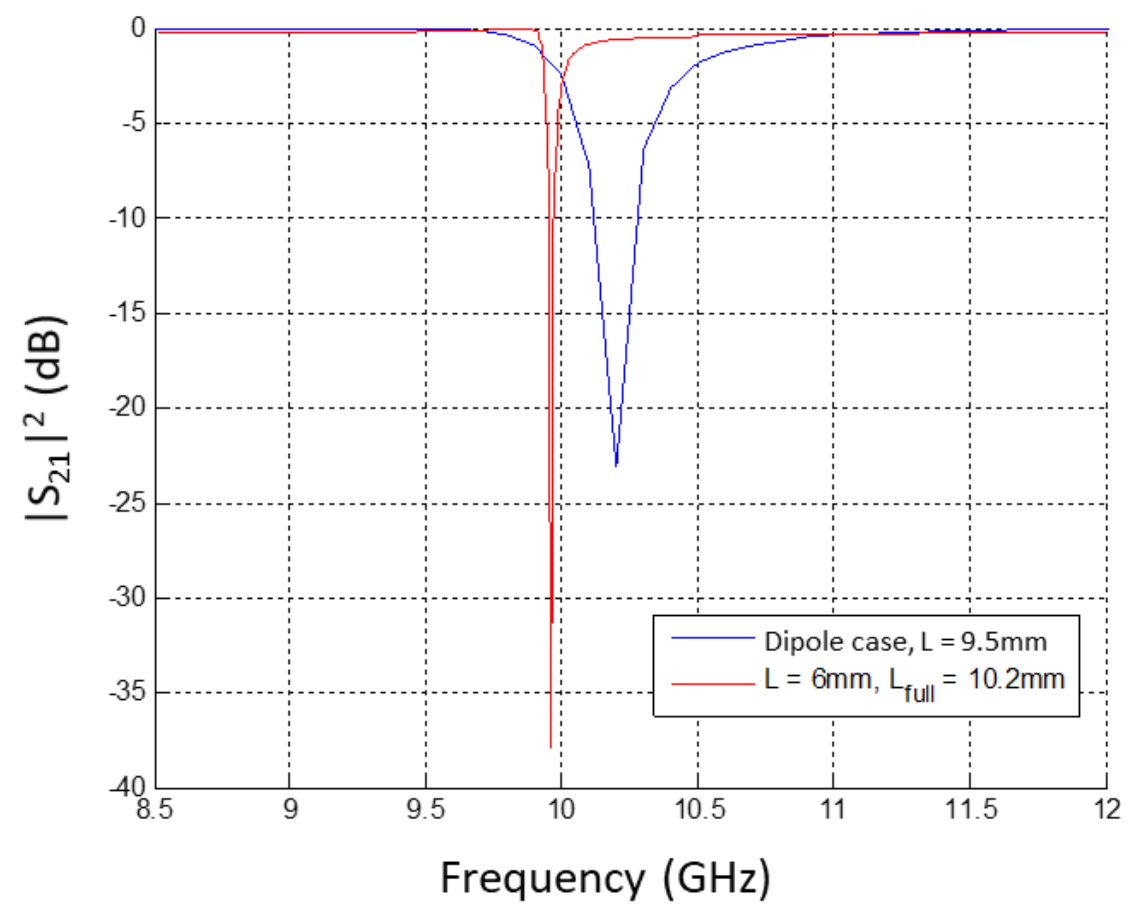

Figure 11. Transmission response demonstrating improved selectivity for bent dipole element (relative to simple dipole).

As seen from the simulation results, the bent dipole design produces several advantages relative to the simple dipole. First, since it utilizes the same periodicity in the 
$x$ - and $y$-directions, the bent dipole produces a lower profile design. That is, the FSS thickness is reduced from $9.5 \mathrm{~mm}$ for the simple dipole to $6 \mathrm{~mm}$ for the bent dipole. This implies $\sim 40 \%$ smaller thickness for a comparable rejection frequency, a significant benefit when trying to reduce the SWaP requirements (as well as the cost) for fabricated designs that are implemented into systems.

However, the primary advantage of using the bent dipole element is that it produces a much narrower rejection band. Specifically, the $-10 \mathrm{~dB}$ relative bandwidth for the bent dipole is $0.15 \%$, demonstrating significantly improved selectivity relative to the simple dipole $(0.77 \%)$. As shown in the graph, this improved selectively also yields sharper roll-offs and improved passband response. Given this narrowband response, the bent dipole design is a good option for applications that require notch filters with highly selective rejections.

\subsection{Bandwidth Control}

In addition to the design parameters for the FSS element itself, the other unit cell parameters can be carefully selected to obtain a desired frequency response. The selection of substrate permittivity and thickness is generally influenced by the specific application or design requirements, as well as by what materials may be available in stock for eventual fabrication. These parameters can also help control the rejection bandwidth. Using the bent dipole design from the previous section, we conducted a parametric analysis to explore the impact on the $-10 \mathrm{~dB}$ rejection bandwidth. The parameters we studied were (1) PEC element width and (2) dielectric substrate thickness. 


\section{PEC Element Width}

The first parameter we analyzed for its impact on bandwidth was the PEC element width, defined as shown in Figure 12.

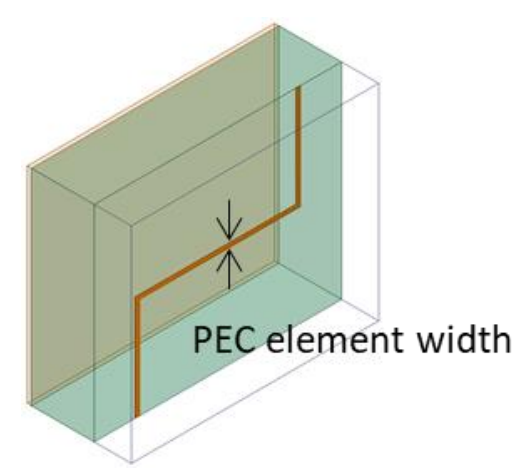

Figure 12. PEC element width for bent dipole design.

Figure 13 explores the impact that varying the PEC element width has on the rejection bandwidth, displaying the $S_{11}$ parameter in Figure 13(a) and the $S_{21}$ parameter in Figure 13(b). The PEC element width was varied from $0.1 \mathrm{~mm}$ to $0.5 \mathrm{~mm}$. Upon close examination, we see that both the transmission $\left(S_{21}\right)$ zeros and reflection $\left(S_{11}\right)$ zeros play an important role in controlling the bandwidth. 


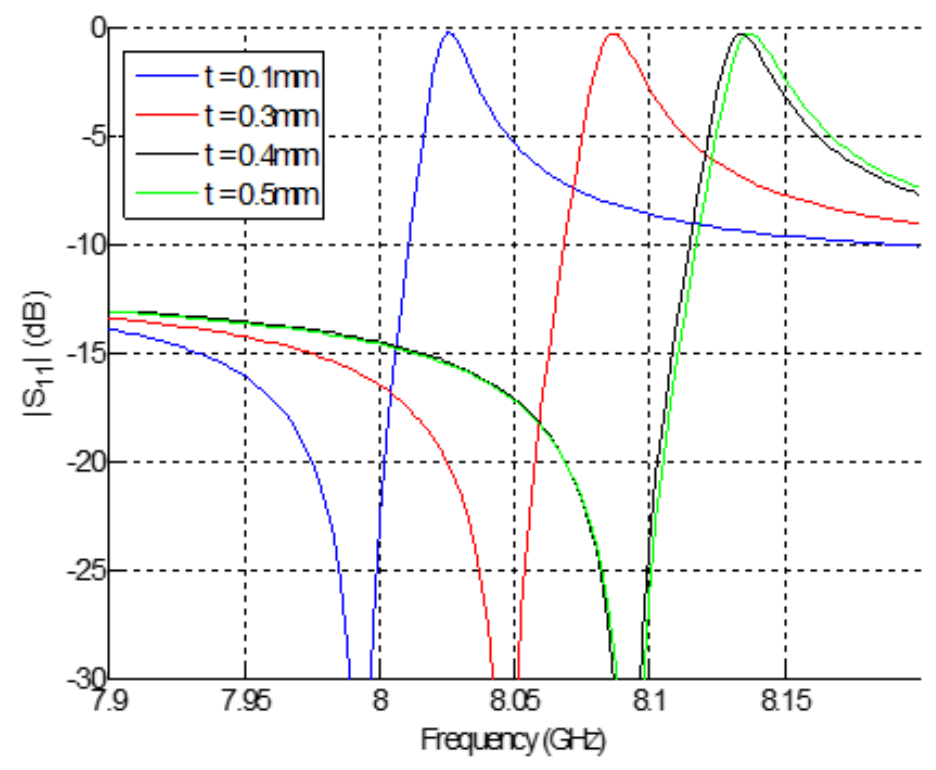

(a)

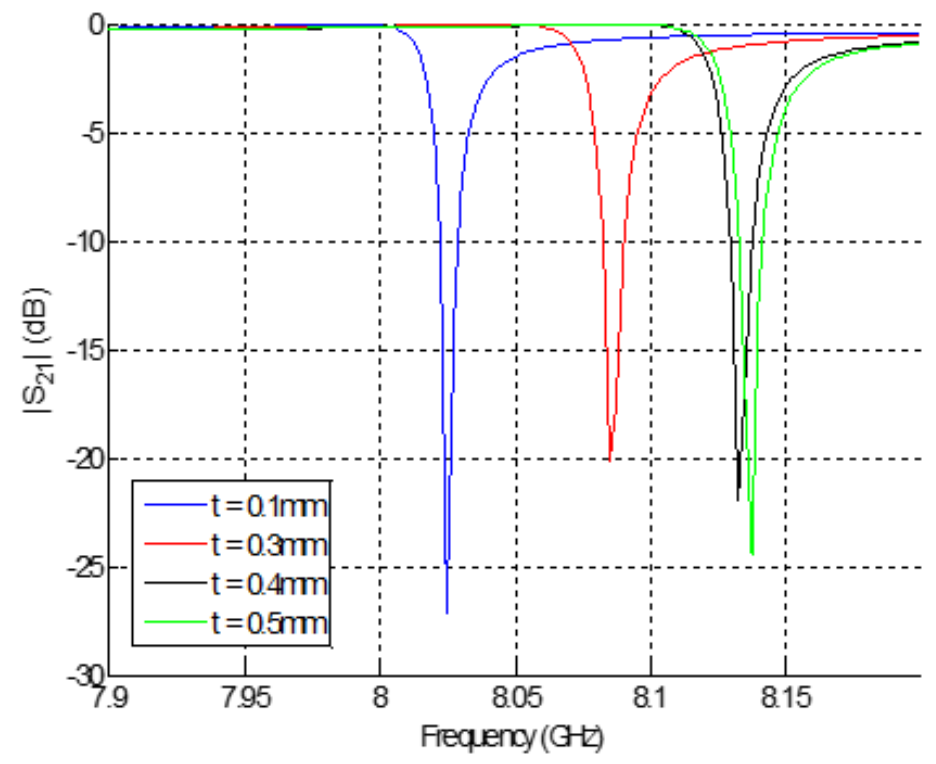

(b)

Figure 13. Performance for varying PEC element widths: (a) $S_{11}$ and (b) $S_{21}$. 
The reflection zeros occur at frequencies where the electrical length of the resonator (i.e. substrate path or air path) becomes a multiple of $\pi$, and these zeros are notably useful in controlling the roll-offs and passband [77, 78]. The PEC element width provides fine tuning to control the location of these reflection zeros. In agreement with microstrip line theory, increasing the element width is found to increase the relative bandwidth (as shown in Table 1) - an effect that remains consistent as dipole width is varied. This is explained in the last column in the table, which demonstrates that a narrower dipole width produces an $\mathrm{S}_{11}$ response with reflection zeros that are more tightly spaced near their respective transmission zeros.

Table 1. Impact of varying dipole width on relative bandwidth.

\begin{tabular}{|c|c|c|c|}
\hline $\begin{array}{c}\text { PEC } \\
\text { element } \\
\text { width }\end{array}$ & $\begin{array}{c}\text { Rejection } \\
\text { frequency }\end{array}$ & $\begin{array}{c}\text { Relative -10dB } \\
\text { bandwidth }\end{array}$ & $\begin{array}{c}\text { Frequency shift from } \\
\text { reflection zero to respective } \\
\text { transmission zero }\end{array}$ \\
\hline $0.1 \mathrm{~mm}$ & $8.0075 \mathrm{GHz}$ & $0.082 \%$ & $32.5 \mathrm{MHz}$ \\
\hline $0.3 \mathrm{~mm}$ & $8.086 \mathrm{GHz}$ & $0.093 \%$ & $38.5 \mathrm{MHz}$ \\
\hline $0.4 \mathrm{~mm}$ & $8.133 \mathrm{GHz}$ & $0.101 \%$ & $41.0 \mathrm{MHz}$ \\
\hline $0.5 \mathrm{~mm}$ & $8.137 \mathrm{GHz}$ & $0.108 \%$ & $45.0 \mathrm{MHz}$ \\
\hline
\end{tabular}

This characteristic is ideal for the narrowband case as sharper roll-offs are created in the $S_{21}$ response, enabling rapid transition from rejection band to passband. Additionally, the rejection band shifts to higher frequencies as the element width is increased. While this frequency response appears to converge and stabilize at larger 
widths (i.e. $0.4 \mathrm{~mm}$ to $0.5 \mathrm{~mm}$ ), the relative bandwidth continues to increase. Overall, element width provides good control over the frequency rejection bandwidth. For a narrowband design, a narrow element width helps achieve sharp roll-offs.

\section{Dielectric Substrate Thickness}

We also studied the thickness of the dielectric substrate in terms of impact on rejection bandwidth. This substrate thickness is defined in Figure 14.

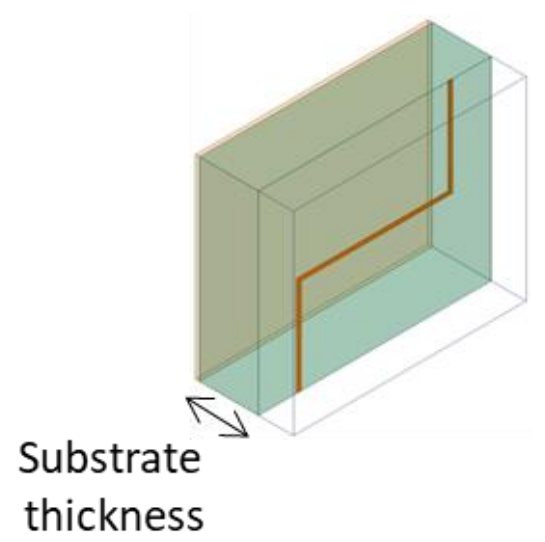

Figure 14. Substrate thickness for FSS unit cell.

Figure 15 displays the transmission response for varying the substrate thickness, using the initial case of $1.524 \mathrm{~mm}$ and then both adding and subtracting $0.25 \mathrm{~mm}$ to explore the impact. Table 2 provides a detailed analysis of this data, including both the rejection frequency and the relative $-10 \mathrm{~dB}$ rejection bandwidth. 


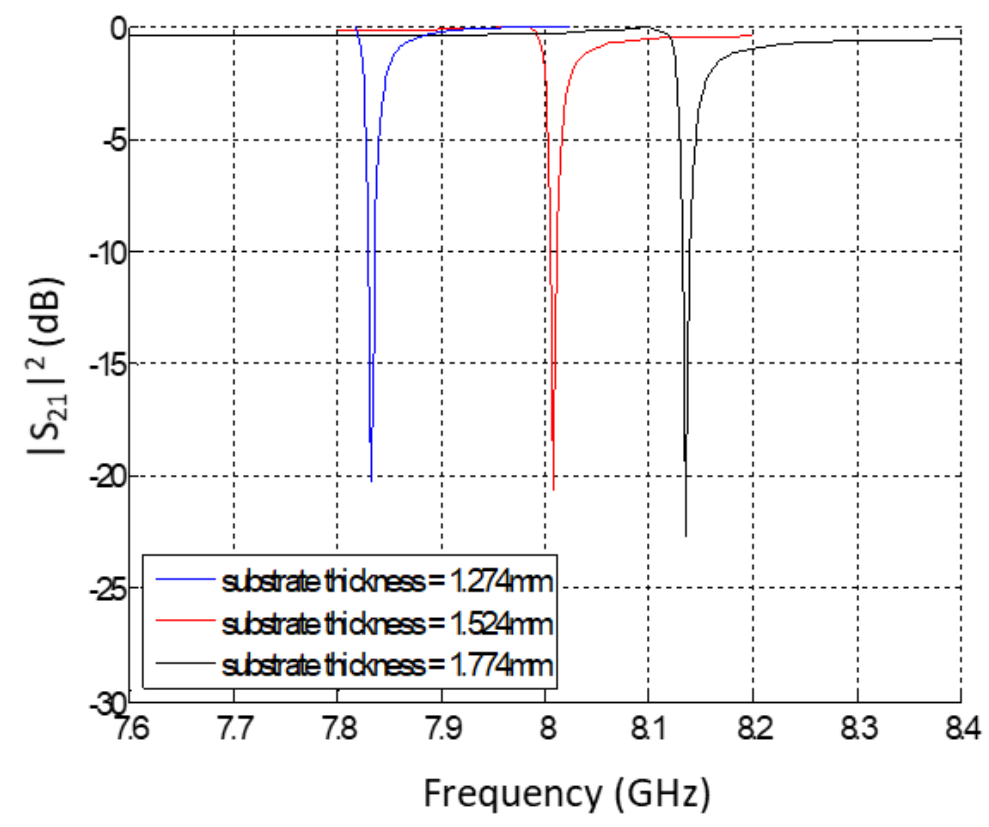

Figure $15 . \mathrm{S}_{21}$ performance for varying dielectric substrate thickness.

Table 2. Impact of varying dielectric substrate thickness on relative bandwidth.

\begin{tabular}{|c|c|c|}
\hline $\begin{array}{c}\text { Substrate } \\
\text { thickness }\end{array}$ & $\begin{array}{c}\text { Rejection } \\
\text { frequency }\end{array}$ & $\begin{array}{c}\text { Relative -10dB } \\
\text { bandwidth }\end{array}$ \\
\hline $1.274 \mathrm{~mm}$ & $7.8325 \mathrm{GHz}$ & $0.080 \%$ \\
\hline $1.524 \mathrm{~mm}$ & $8.0075 \mathrm{GHz}$ & $0.082 \%$ \\
\hline $1.774 \mathrm{~mm}$ & $8.135 \mathrm{GHz}$ & $0.085 \%$ \\
\hline
\end{tabular}

Since the incident wave propagates along the length of the element for a thick FSS, the dielectric substrate thickness has minimal impact on bandwidth for narrowband designs - notably different from its impact on planar frequency selective surfaces. However, we should note that for wideband designs, thicker substrates are known to result 
in increased coupling to higher-order modes [79]. This enables control of multiple transmission zeros to create a wider rejection band.

\subsection{Extension to Band-pass Designs}

While this research primarily focuses on band rejection for thick FSSs, we understand that there are just as many practical applications for band-pass designs. We therefore conducted a brief study to explore the extension to these designs. By simply reversing the role of PEC and dielectric on the surface of the unit cell element (see Figure 16), we create a slot design. As the dual of the patch design, we produce a bandpass response at the exact same frequency as for the rejection from the traditional bent dipole design.

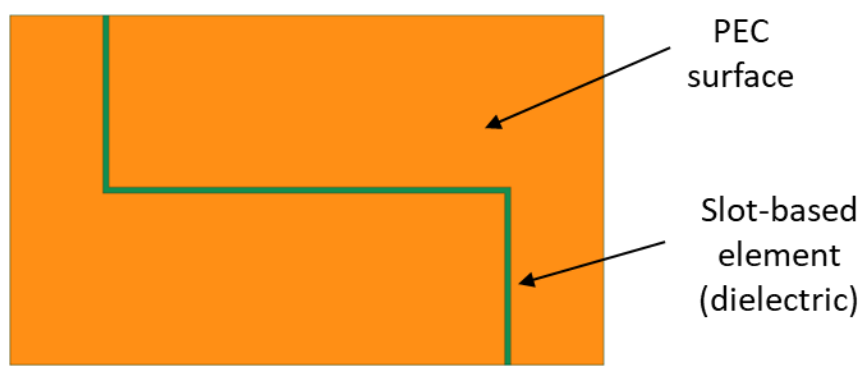

Figure 16. Slot design for band-pass response.

While traditional planar band-pass FSS designs often display low selectivity, thick FSS principles can again overcome these limitations. We see the $S_{21}$ response for several different $L$ values in Figure 17, with the highlighted design (for $L=6 \mathrm{~mm}$ ) achieving an 
impressive $0.91 \%$ relative $-3 \mathrm{~dB}$ bandwidth. These results are for an incident wave with the magnetic field oriented with a perpendicular polarization with respect to the element.

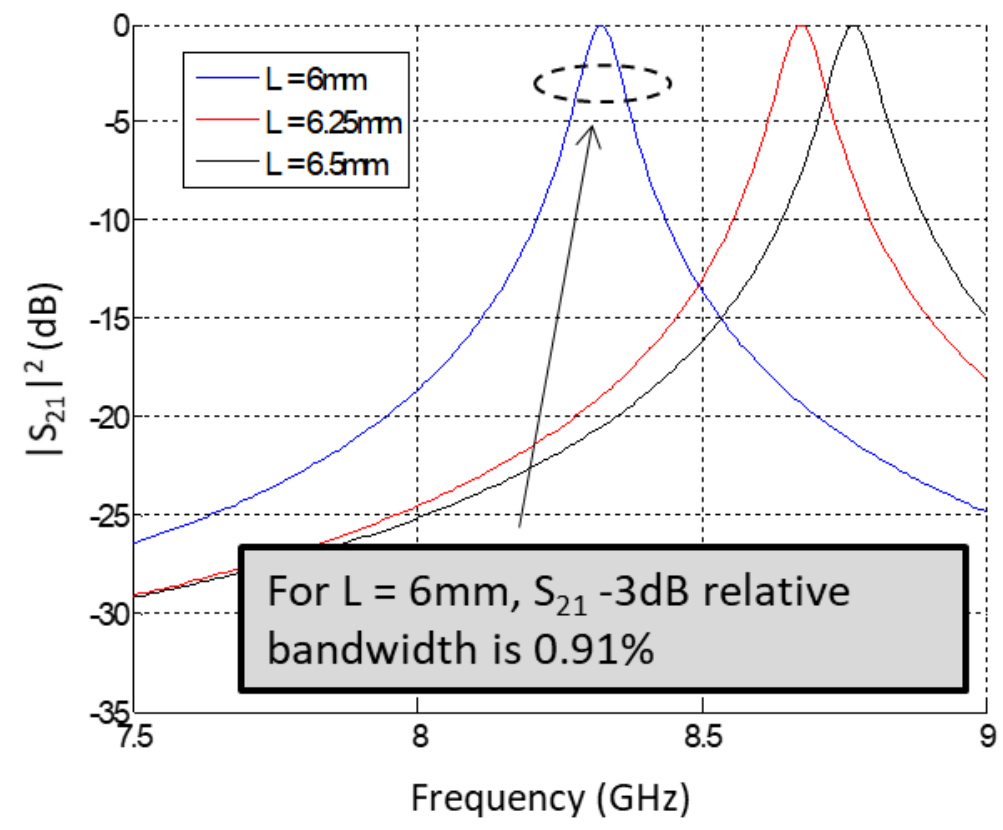

Figure 17. $S_{21}$ performance for slot design producing band-pass frequency response.

Similar to our band rejection designs, the band-pass designs demonstrate high selectivity and a narrowband performance. Band-pass designs allow the FSS to be used as a reconfigurable cover layer for multi-function applications, as well as in frequency switching for communication and/or signal security. Although there is interest in both types of designs, our focus will remain on band rejection FSSs as we explore novel designs for dynamic frequency reconfiguration. Due to the sharp roll-offs and improved 
selectivity, the bent dipole serves as the basis for our early designs - and forms the foundation for our initial research into reconfigurable FSS elements. 


\section{Chapter 4. Frequency Reconfiguration}

Having achieved a thick FSS design with excellent selectivity, our next objective was to build upon this design to explore frequency reconfiguration and agility within a single design. Reconfigurability is a key aspect of future thick FSS designs, and there is much room for innovation and further development in this area. This research introduces a novel RF reconfiguration technique that is designed and analyzed for UWB antennas, by expanding on the thick FSS concept. Dynamic frequency agility will provide the necessary means for 1) adjusting an antenna's frequency of operation, 2) spectrum management, 3) handling of multi-load apertures, and 4) communication security. We explored different thick FSS designs, with a focus on controlling the frequency response for narrowband rejection applications by dynamically adjusting the FSS geometry. Further, these designs can be multi-band for multi-function notch filter applications, such as STAR capability with the potential to improve device efficiency rather drastically [73]. Previous designs demonstrated multi-band rejections for planar surfaces, but did not enable tunable control of the frequency band via geometry reconfiguration [80-83].

These thick FSS designs can then be incorporated into systems, e.g. as reconfigurable cover layers for wideband antennas, and for high band selectivity to achieve isolation and interference reduction. This will help fill the current and future 
demand for RF front-ends that are frequency agile, wideband, reconfigurable, very small in size and weight, and of low power - a necessity for future designs.

\subsection{Reconfigurable Multi-Arm Bent Dipole}

During the early stages of the research, a key accomplishment was a demonstration that the proposed bent dipole element design produces several important advantages, notably a narrower rejection band, a significantly lower profile, and greater control of the rejection band location and bandwidth. As such, this bent dipole design served as the foundation for the unit cell for our initial analysis of reconfiguration and highly selective frequency control for tunable thick FSSs. By using this element as a starting point, we analyzed several designs using the FSS as a band rejection filter.

Our novel approach introduces a new class of thick FSS elements that achieves frequency reconfiguration, by altering the physical geometry of a thick FSS element to achieve dynamic control within a single unit cell design. The initial design concept expanded the thick FSS concept into a multi-arm design depicted in Figure 18. As is shown, the new FSS element is based on the bent dipole concept. The top portion of the bent dipole is created using the PEC strip labeled $\mathrm{L}_{0}$. From there, four different arms (labeled $\mathrm{L}_{1}, \mathrm{~L}_{2}, \mathrm{~L}_{3}$, and $\mathrm{L}_{4}$ ) are used to create four distinct pathways to complete the bottom portion of the bent dipole. Each of these four arms is an individual strip, separated from the other PEC segments by a gap. The set of horizontal strips is designed to connect the various arms to complete the bent dipole. 


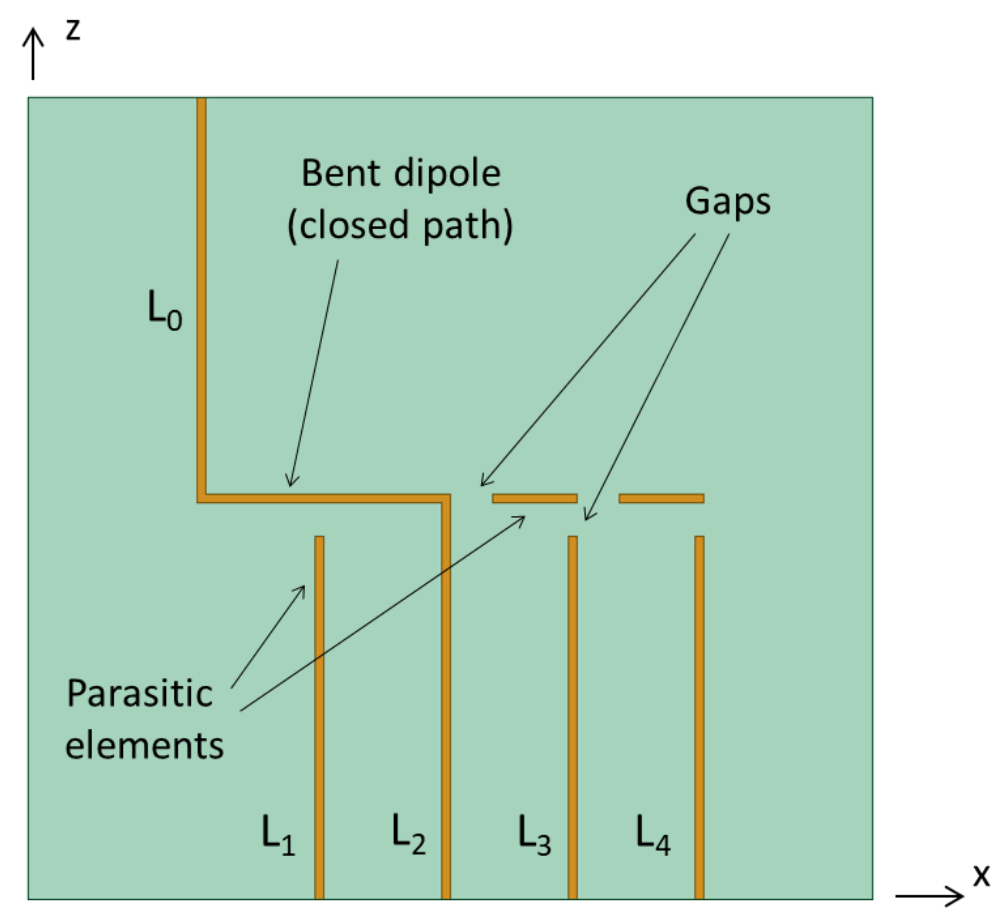

Figure 18. Multi-arm bent dipole element for reconfigurable frequency control.

For simulation purposes, this element is modeled using gaps to separate the various element strips - thus keeping the bent dipole apart from the remaining open paths. For a given simulation, the gap is then "closed" by completing the strip, in effect creating a single bent dipole (e.g., the path created by $\mathrm{L}_{2}$ in Figure 18). The three other arms remain unconnected, simply serving as parasitic elements. While they will create their own frequency rejections, their lengths are such that they will resonate well outside the frequency range of interest. While the simulations model the element using distinct gaps and strips, in practice this design would require switching devices to open or close the gaps. By using this multi-arm FSS element, we can adjust the length of the bent dipole within a single multi-function design. The four arms are used to vary the length, 
enabling reconfiguration to reject four different frequency bands by choosing which of the paths to close. The final design is simply a collection of individual bent dipoles (of varying lengths) combined into a single unit cell structure.

A more thorough description of the multi-arm element unit cell and FSS array is shown in Figure 19. Here we clearly see the $L_{\text {full }}$ and $L$ parameters, and how they relate to the multi-arm design. While $L$ remains constant for each of the four paths, we are able to choose four distinct $L_{\text {full }}$ values to control the rejection frequency.

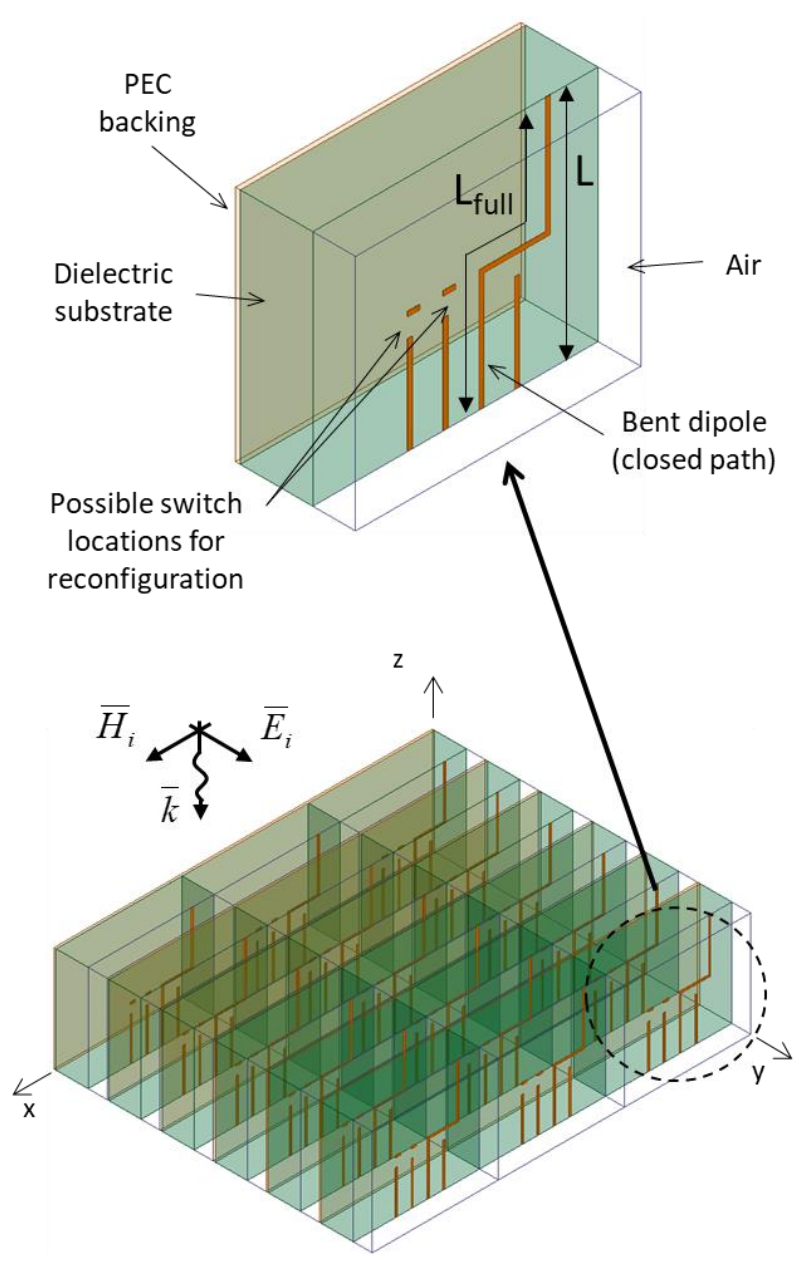

Figure 19. Multi-arm bent dipole unit cell and FSS array. 
The tunable multi-arm bent dipole concept was modeled via full-wave approaches. Through this reconfigurability study, the incident wave was assumed to be at normal incidence. Further, early in the process we made a slight adjustment to the element shown in Figure 18, such that the values for $\mathrm{L}_{1}$ through $\mathrm{L}_{4}$ were set to be different from one another. Specifically, $\mathrm{L}_{1}<\mathrm{L}_{2}<\mathrm{L}_{3}<\mathrm{L}_{4}$ in order to more rapidly alter $L_{\text {full }}$ from the smallest value (the bent dipole created using $L_{1}$ ) to the largest value (the bent dipole created using $\mathrm{L}_{4}$ ), thus enabling a wider tunable frequency range within a compact single-element design. This new ramped bent dipole design is depicted in Figure 20.

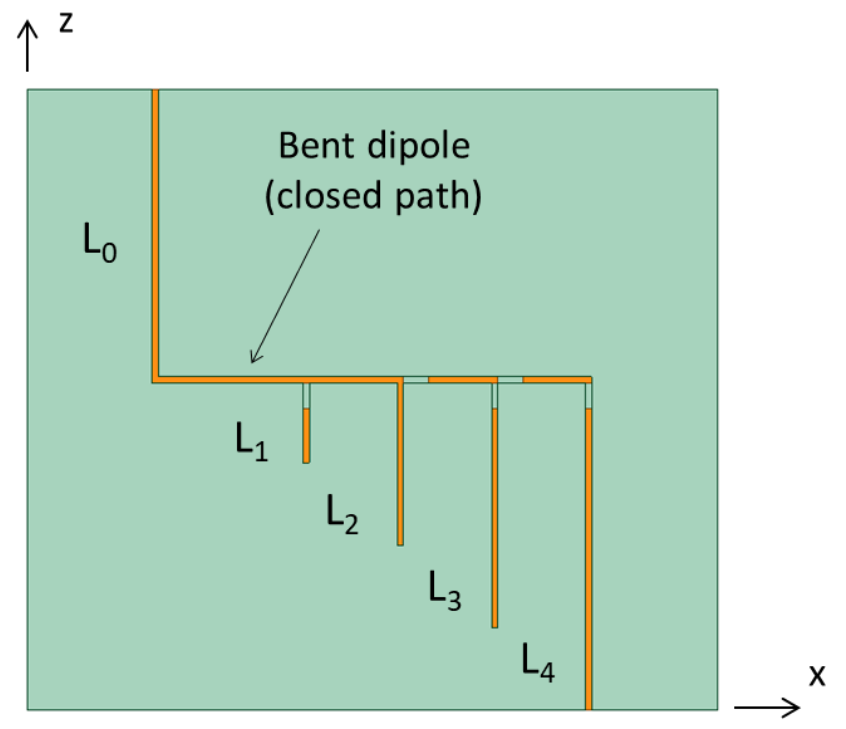

Figure 20. Ramped multi-arm bent dipole element.

As a design exercise, we aimed to use the bent dipole design principles discussed in Chapter 3 to create a tunable FSS design that operates across the 6-12 GHz frequency 
range. To determine values for $L_{f u l l}$, we start by estimating the shortest value using $12 \mathrm{GHz}$. Here, the free-space wavelength $\left(\lambda_{0}\right)$ is $25 \mathrm{~mm}$. Using a relative dielectric permittivity of 3.38 , the guided wavelength is calculated from $\lambda_{g}=\lambda_{0} / \sqrt{\left(\varepsilon_{r}+1\right) / 2}$, resulting in an $L_{f u l l}$ estimate of $\lambda_{g} / 2=8.45 \mathrm{~mm}$. Using the same process to calculate the maximum value from $6 \mathrm{GHz}$, we get $L_{f u l l}=16.89 \mathrm{~mm}$. To design for tunability from 6-12 GHz, we set $L_{f u l l}=8.45 \mathrm{~mm}, 11.26 \mathrm{~mm}, 14.08 \mathrm{~mm}$, and $16.89 \mathrm{~mm}$ using the $\mathrm{L}_{1}, \mathrm{~L}_{2}, \mathrm{~L}_{3}$, and $\mathrm{L}_{4}$ bent dipoles, respectively. The gap sizes were all set to $0.5 \mathrm{~mm}$, and the arms were equally spaced in the horizontal direction. The PEC strip thickness was $0.1 \mathrm{~mm}$, the dielectric thickness was $1.524 \mathrm{~mm}$, and the air thickness was $1 \mathrm{~mm}$. Finally, the unit cell cross-section was $8 \mathrm{~mm}$ x $8 \mathrm{~mm}$.

Figure 21 compares the $S_{21}$ performance for each of these 4 cases. This FSS design was demonstrated for wideband operation across $6 \mathrm{GHz}$ to $12 \mathrm{GHz}$. We immediately see that our $L_{f u l l}$ estimates produce the desired tunable response, with the rejection frequency varying very precisely across this range. As expected, choosing arm lengths that keep the overall bent dipole length between $8.45 \mathrm{~mm}$ and $16.89 \mathrm{~mm}$ produce rejections within this frequency range. Because of the ramped design for the four arms, we rapidly transition across this wide dynamic range within the same compact unit cell element. In addition, each rejection band retains the same advantages of the bent dipole element, namely high selectivity that is a significant improvement relative to reconfigurable designs using planar frequency selective surfaces $[50,53,54,60]$. We also note that the parasitic elements have minimal impact on the rejection frequencies and performance. 


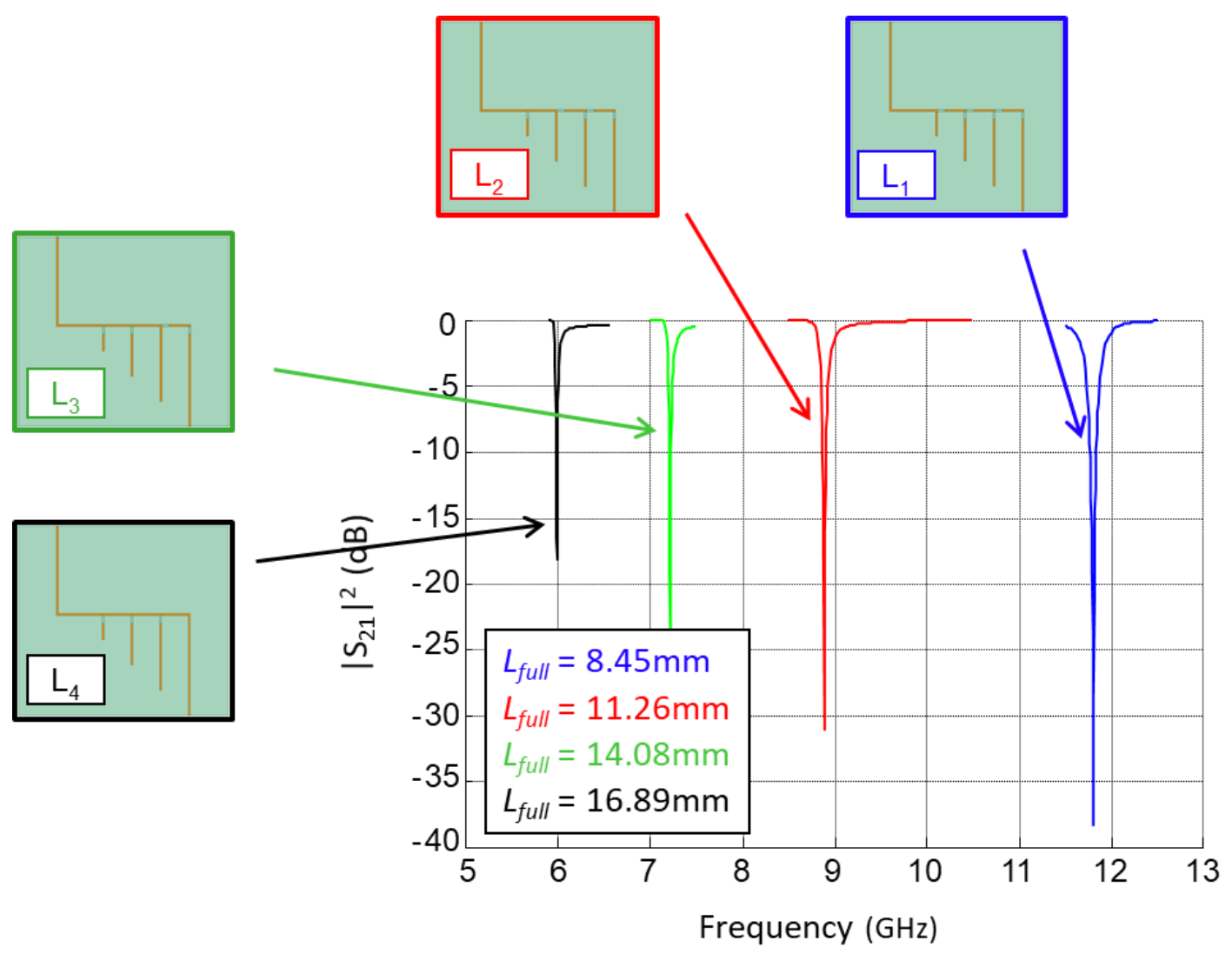

Figure 21. Frequency control and reconfiguration across 6-12 $\mathrm{GHz}$ using ramped multi-arm bent dipole FSS.

As expected from microstrip line theory, higher-order resonances will occur for each of these rejections [84]. Figure 22 demonstrates this behavior. As shown on the right of the graph, the bent dipole created from the $\mathrm{L}_{4}$ arm (with a rejection around 7.5 GHz) has a higher-order rejection near $15 \mathrm{GHz}$. Such higher-order resonances can be designed to occur outside the frequency range of interest with high precision due to the sharp roll-offs. To avoid higher-order resonances overlapping with designed rejections, the bent dipoles should be designed such that the $L_{\text {full }}$ for the longest arm is less than twice the $L_{\text {full }}$ of the shortest arm. In the case of Figure 22, this means ensuring that 
$L_{4, \text { full }}<2 L_{1, \text { full }}$ when selecting the parameter values. With this stepped multi-arm FSS element, we can adjust the length of the bent dipole using a single multi-function design.

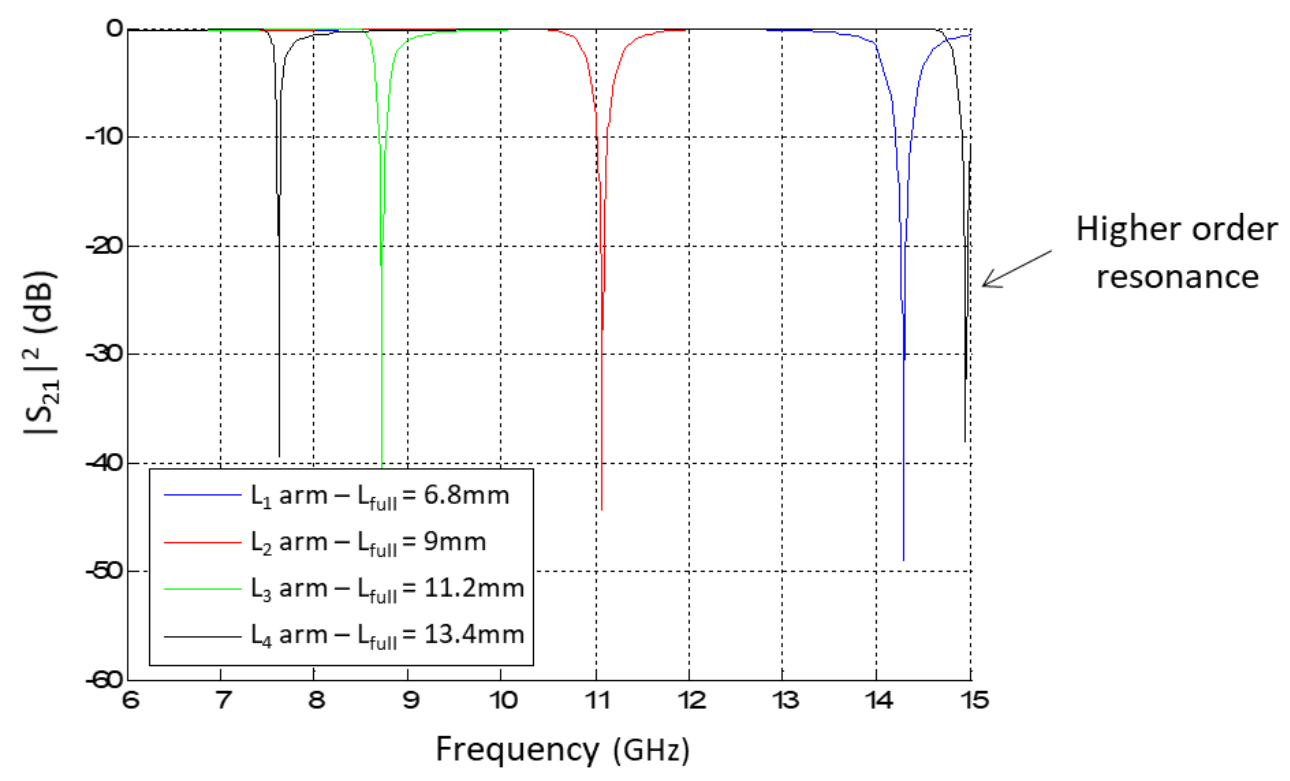

Figure 22. Higher-order resonances for ramped multi-arm dipole FSS.

\subsection{Reconfigurable Angled Dipole}

Even though the simulated designs have not yet incorporated actual switching elements, they will eventually be required for dynamic frequency reconfiguration. Since these components will add to the cost, weight, complexity, and power requirements of a final design, we must optimize their usage and placement. Therefore, by building upon 
the ramped multi-arm element, we next analyzed a modified design to reduce the required number of switching elements. This new element design is displayed in Figure 23.

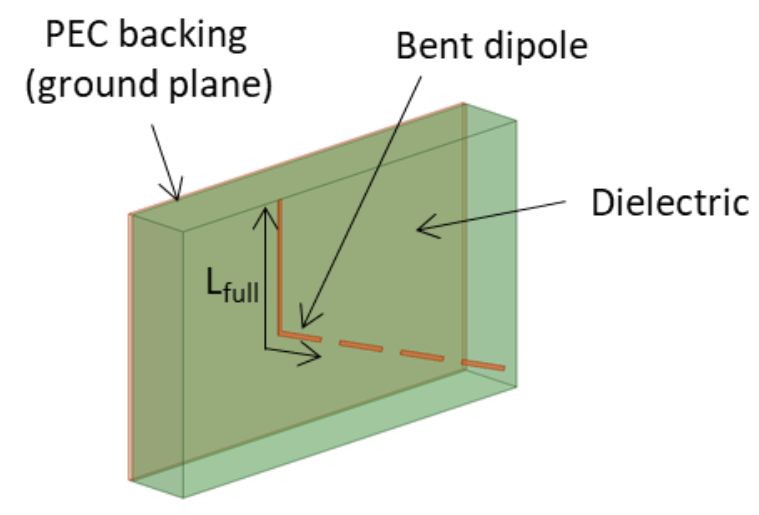

Figure 23. Thick FSS unit cell for reconfigurable angled dipole.

As before, the gaps can be successively closed to increase the length of $L_{f u l l}$, and thus reconfigure the geometry to shift the rejection frequency. The new design exhibits a significant reduction in the number of switching elements required. For the current example of a 4-way reconfigurable design, the new element reduces the number of switching elements to 3 - versus the 7 required for the element in Figure 20. This efficiency is a critical step toward design optimization, and with it an ultimate reduction in the cost, weight, complexity, and power requirements.

This design also retains the attractive features from the initial bent dipole design. Specifically, the design continues to demonstrate (a) high selectivity and (b) low profile. Table 3 displays the rejection frequency and relative $-10 \mathrm{~dB}$ rejection bandwidth for various cases where $L_{\text {full }}$ ranges from $4.40 \mathrm{~mm}$ to $10.25 \mathrm{~mm}$. For this design, the exact 
same unit cell dimensions were used as with the original bent dipole design, and therefore the same low profile benefits are achieved. And most importantly, the highly selective rejections are maintained, with relative $-10 \mathrm{~dB}$ bandwidth varying between $0.26-0.79 \%$.

Table 3. Rejection frequency and relative bandwidth for angled dipole FSS design.

\begin{tabular}{|c|c|c|c|c|}
\hline & \multicolumn{2}{|c|}{ Shortest dipole } & \multicolumn{2}{|c|}{ Longest dipole } \\
\hline $\mathrm{L}_{\text {full }}(\mathrm{mm})$ & 4.40 & 6.35 & 8.30 & 10.25 \\
\hline Rejection freq (GHz) & 21.28 & 15.06 & 11.75 & 9.71 \\
\hline Relative $-10 \mathrm{~dB}$ BW & $0.79 \%$ & $0.56 \%$ & $0.37 \%$ & $0.26 \%$ \\
\hline
\end{tabular}

\subsection{Multi-band Designs}

With a goal of incorporating these thick FSS designs into systems for multifunction applications, we extended this frequency control to multi-band designs. To this end, we interleave two different side-by-side elements (each designed to reject unique frequencies) to produce multiple rejections within the same unit cell.

To study the basic multi-band performance and characteristics, we returned to the simple dipole for a quick proof of concept. We created an array by interleaving two different dipoles in an alternating arrangement, maintaining one dipole at the same $L=9.5 \mathrm{~mm}$ from [18] and adjusting the length of the second dipole. The $\mathrm{S}_{21}$ performance is shown in Figure 24. As expected, when the second dipole is also set to $L=9.5 \mathrm{~mm}$, the 
results converge to the case of the single dipole. However, as the length of the second dipole is reduced, there are multiple rejections produced - one at the original rejection frequency, and one at a higher frequency. Notably, adding the second resonance has minimal effect on the location of the rejection frequency produced by the original dipole. Finally, by alternating the two dipole designs to create multiple notches, the sharp rolloffs enable precise tuning to alter the passband response and bandwidth.

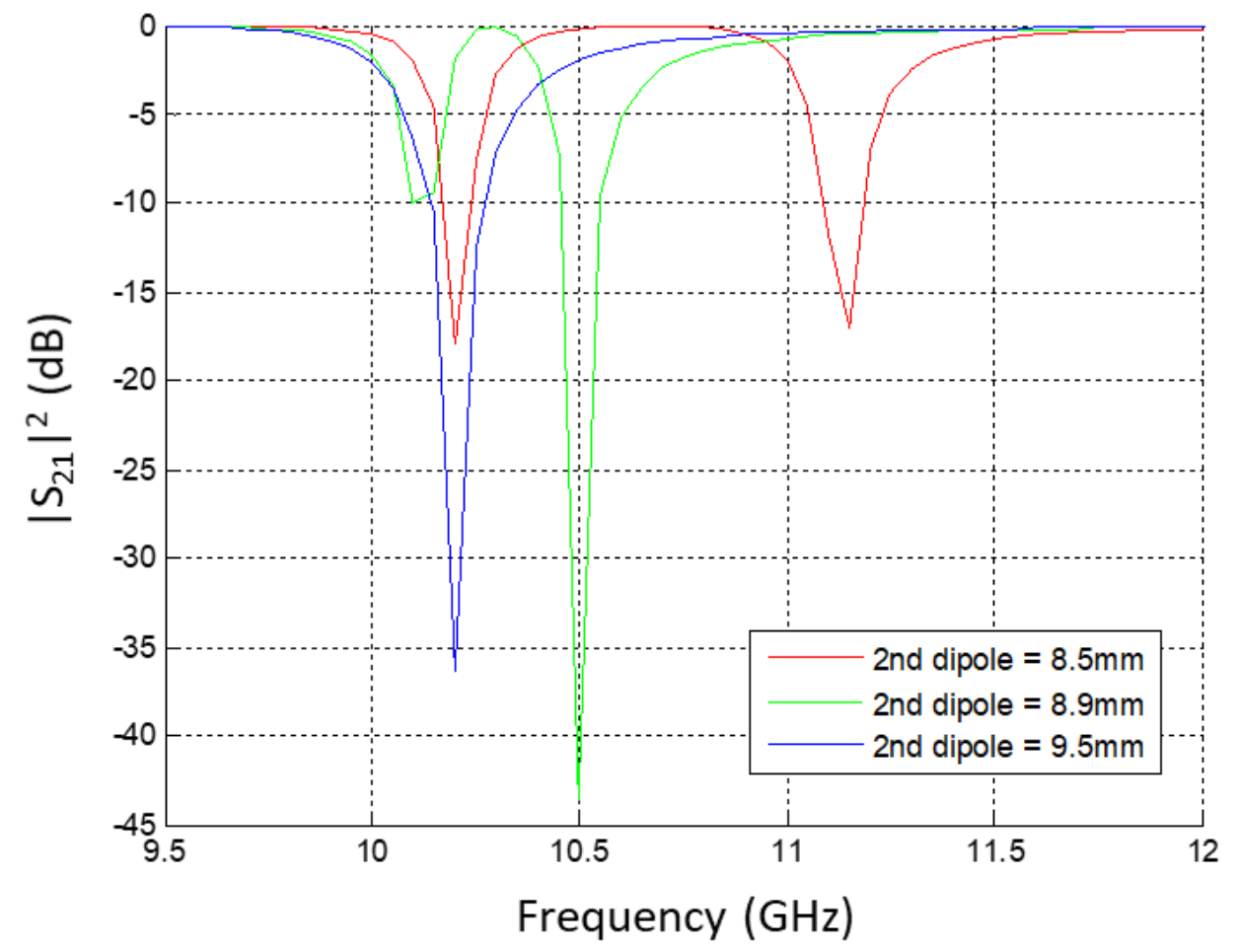

Figure 24. Multiple rejections produced by interleaving two simple dipole designs. 
After establishing this baseline behavior, we used the same principle to create multi-band designs via the multi-arm dipole element. An example design is displayed in Figure 25. With four arms per element, this multi-function design enables 16 different frequency reconfigurations by adjusting which two paths are closed; each reconfiguration produces a dual-band rejection. This adaptability is useful for multi-mode antenna applications.

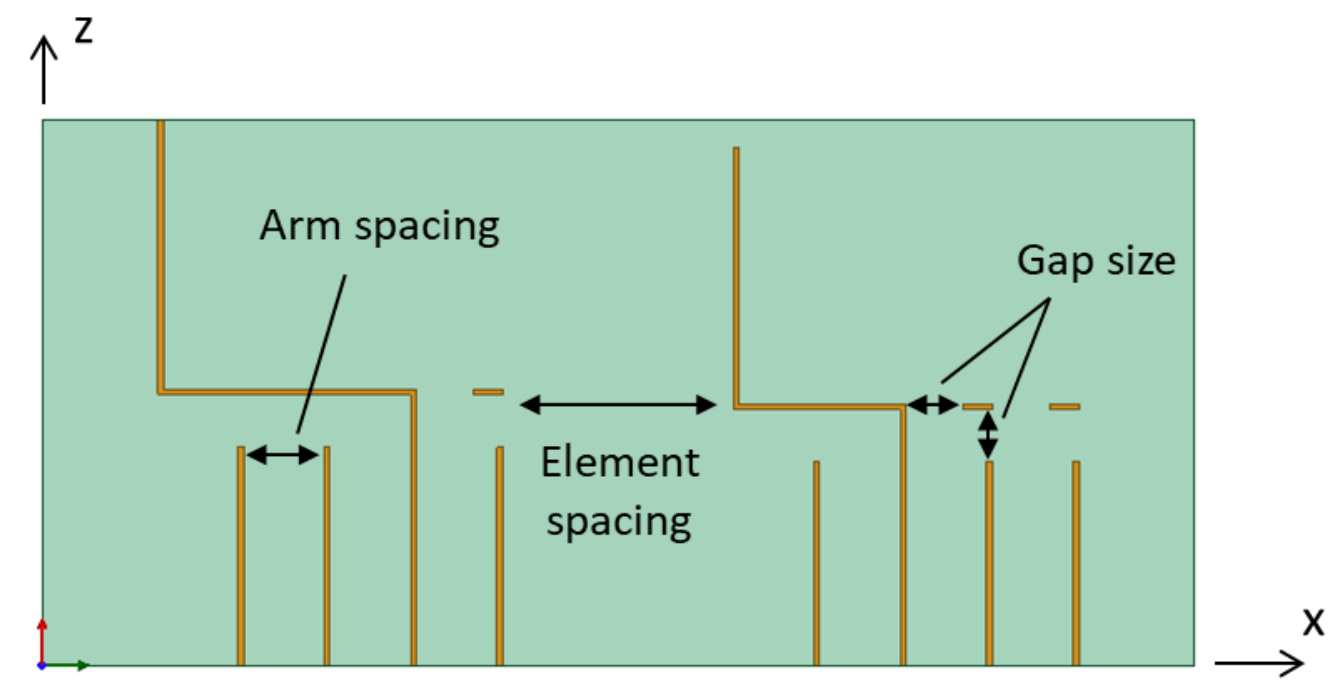

Figure 25. Multi-band unit cell design using interleaved multi-arm bent dipole elements.

As seen in Figure 26, this particular design demonstrates multi-band capability for reconfiguration. By using dipole lengths of $15.45 \mathrm{~mm}$ and $10.45 \mathrm{~mm}$, we create rejections at $6.56 \mathrm{GHz}$ and $9.7 \mathrm{GHz}$, respectively. To reduce unit cell size, design parameters are: gap size $=0.5 \mathrm{~mm}$, arm spacing $=1.5 \mathrm{~mm}$, and element spacing $=2 \mathrm{~mm}$. The sharp rolloffs again enable precise tuning of not only the rejection bands, but also the passband. 
While this example produces 16 dual-band reconfigurations, this concept can be expanded to include any number of reconfigurations or tunable frequency range, simply by altering the multi-arm elements. While this added design flexibility is an advantage, there are also tradeoffs that must be considered when designing for multiple rejections. For instance, since a fabricated FSS array is finite, only a subset of the array elements will be tuned to each frequency. This will in effect weaken the frequency response, an outcome that is not seen in the infinitely periodic designs from simulations.

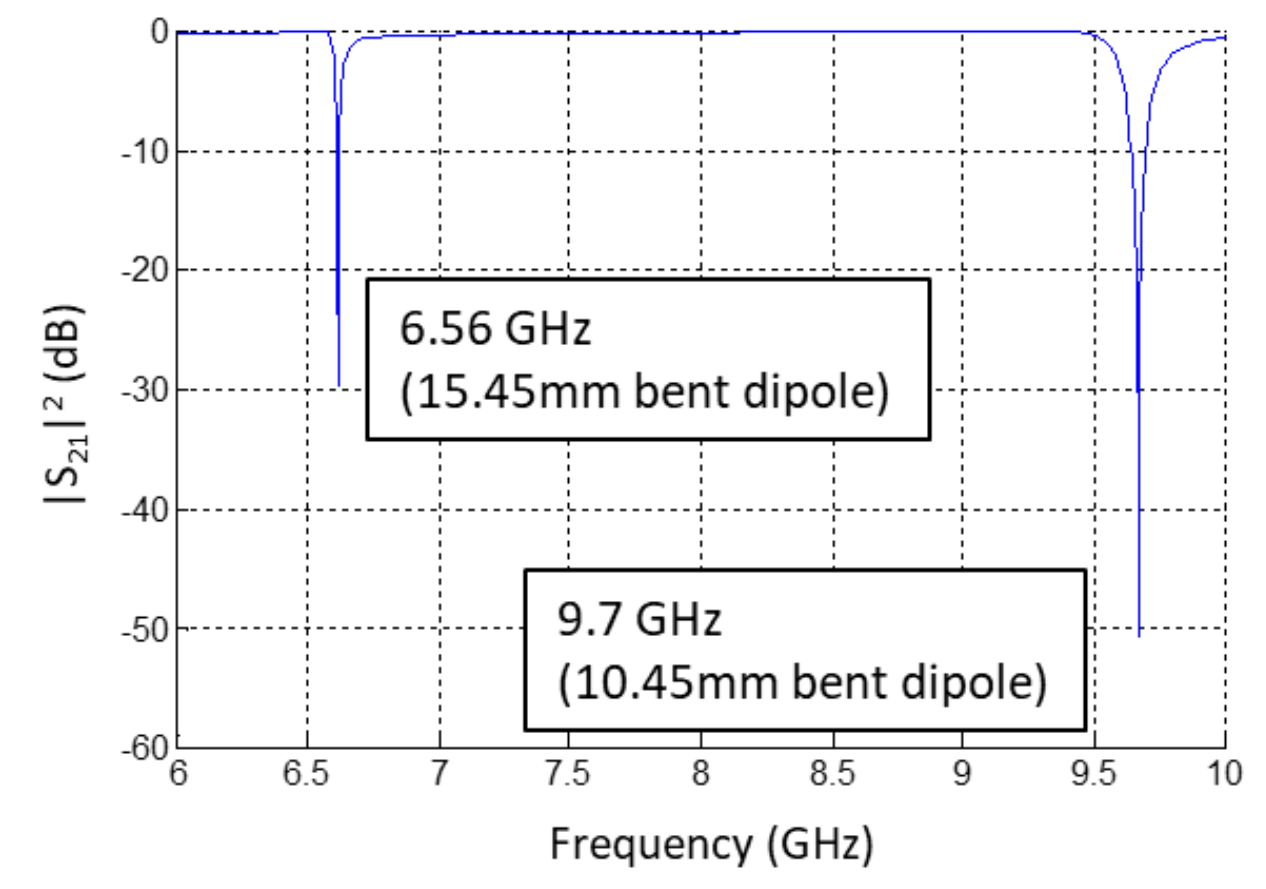

Figure 26. Dual-band rejection response for interleaved multi-arm bent dipoles. 


\section{Parametric Analysis}

As we see from Figure 25, there are several areas where coupling can become a design factor. These include arm spacing, gap size, and element spacing. Figure 27 displays an example of the impact of varying the gap size on the $S_{21}$ frequency response. In this example, we see that the rejection band seems to shift until it converges for a gap size of $1 \mathrm{~mm}$. Similar studies were conducted for arm spacing and element spacing, where each parameter has a measurable impact on rejection frequency that converges as spacing (from the "active" bent dipoles) is increased and coupling is minimized.

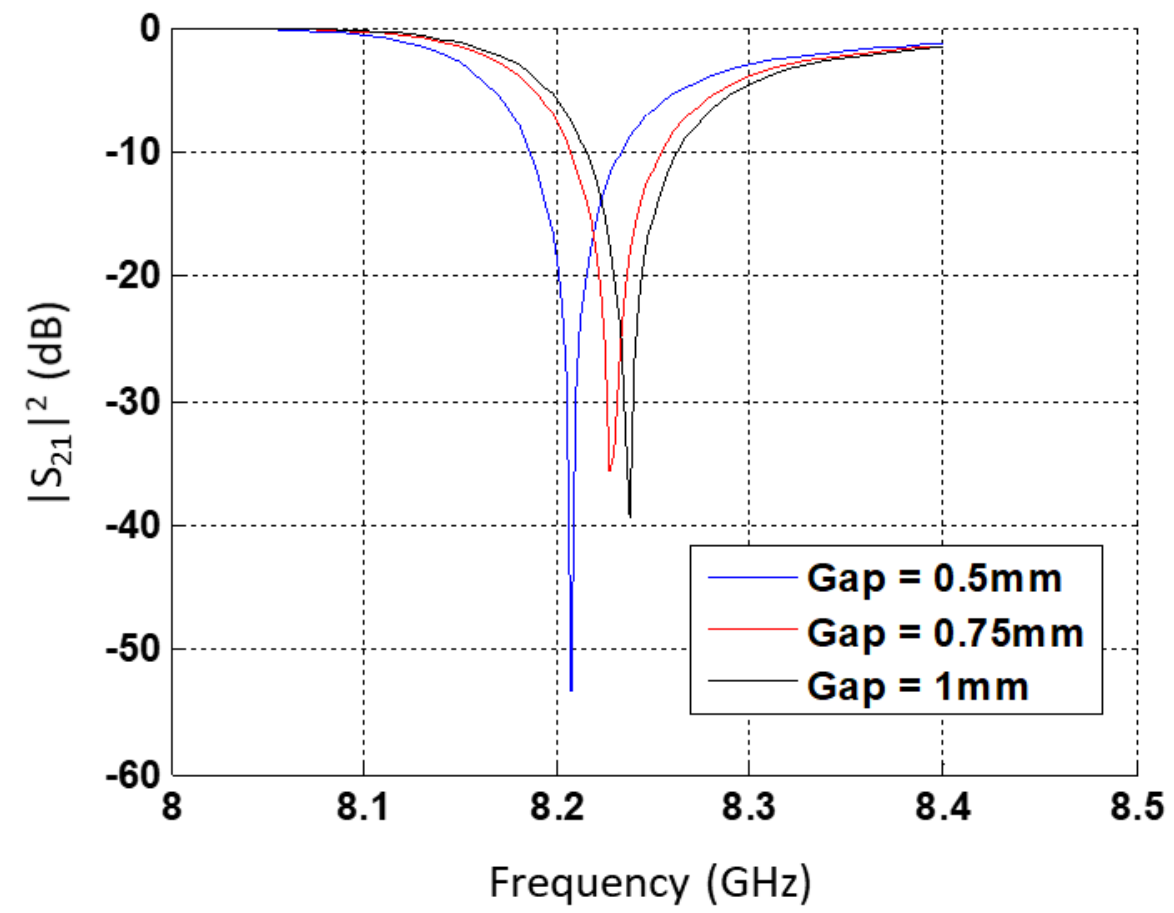

Figure 27. Impact of varying gap size on rejection frequency. 
When all spacings are set to minimize coupling, the rejection frequencies are identical to that of a single bent dipole of the same dimensions. However, this spacing strategy can be overly conservative in preventing practical and compact FSS element designs. Efficient design techniques should increase the number of elements in a finite array by reducing spacing and working with coupling, accepting this tradeoff by making finer adjustments during simulations to determine the impact.

By expanding the thick FSS concept into a novel multi-arm element technique, we combined dynamic frequency reconfiguration with a multi-function band rejection filter design, demonstrating great potential for future systems. Relative to planar surfaces, reconfigurability for thick structures has been much more limited. Nevertheless, our design achieves both wide tunable range and narrow relative bandwidth when compared to other thick FSS designs $[67,70]$. These filters have demonstrated an ability to control and adjust narrowband rejection frequencies within a single multi-function design. However, to this point the simulations have been limited to normally-incident waves. In order to design for practical applications, we must develop FSSs that provide stable performance responses with respect to incidence angle. 


\section{Chapter 5. Performance Stability with Incidence Angle}

Upon analyzing and developing useful techniques for reconfiguration, our focus transitioned to achieving performance stability with respect to incidence angle. To this point, all simulations have been run for normally-incident plane waves. In order to be useful for practical applications, we set a goal of performance stability through incidence angles of at least $30^{\circ}$. This proved to be a significant challenge - particularly for the narrowband designs explored here, since even slight shifts in frequency cause instability in the rejection band.

To explore the impact of incidence angle on performance, we establish a coordinate frame as pictured in Figure 28. Specifically, we see $\theta$ as rotated from the zaxis toward the $\mathrm{x}$-axis, and $\phi$ as rotated from the $\mathrm{x}$-axis toward the $\mathrm{y}$-axis. Incoming plane waves will be referenced as such throughout this study. As mentioned earlier, we will only focus on the polarization shown in Figure 5, since no transmission mode exists when the electric field is parallel to the PEC ground planes. 


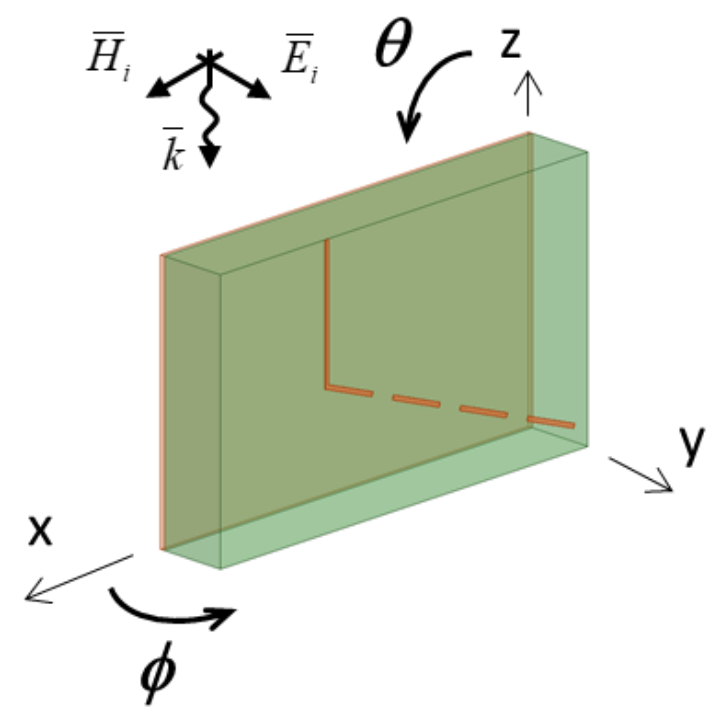

Figure 28. $(\theta, \phi)$ orientations for incidence angle used in FSS design.

\subsection{Bent and Angled Dipole Designs}

In exploring stability with incidence angle, we initially tested a variety of element designs and dimensions. In each of these early designs, we standardized the unit cell to use dielectric permittivity $=3.38$, substrate thickness $=1.524 \mathrm{~mm}$, air thickness $=1 \mathrm{~mm}$, unit cell width $=10 \mathrm{~mm}$, and PEC strip width $=0.1 \mathrm{~mm}$.

Our foundational multi-arm bent dipole design (see Figure 20) suffered from frequency shifts in the rejections band for incidence angles of $15^{\circ}$, so we transitioned our focus to the angled design from Figure 28. This design was simulated for the longest arm length of $L_{f u l l}=10.25 \mathrm{~mm}$ (i.e. when all gaps were closed), and for $\phi=0^{\circ}$. The $S_{21}$ response for this design is shown in Figure 29, by varying $\theta$ from $0^{\circ}$ to $30^{\circ}$. 


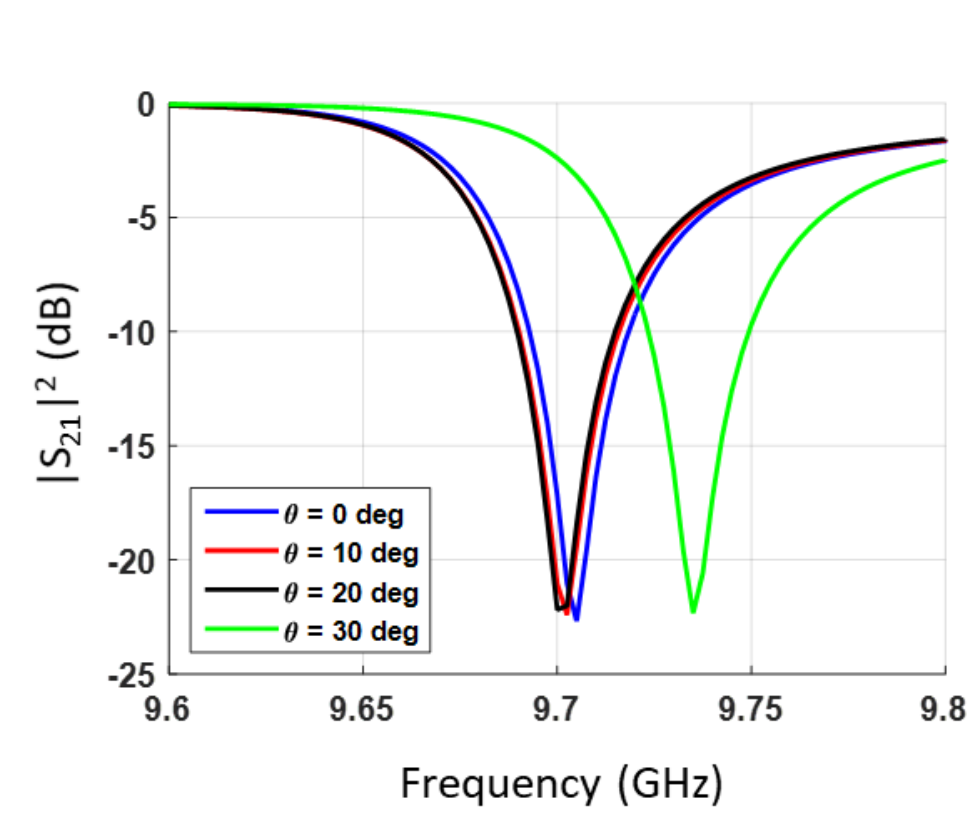

Figure 29. $S_{21}$ performance for angled dipole by varying incidence angle $(\theta)$, for $\phi=0^{\circ}$.

This angled dipole element displays very good stability through $20^{\circ}$, with negligible frequency shifting. This level of stability was promising for an initial attempt, especially given the extremely narrowband nature of the design. However, the $-10 \mathrm{~dB}$ rejection band shifts completely outside the original band for an incidence angle of $30^{\circ}-$ even though this shift is only about $40 \mathrm{MHz}$. We therefore explored additional designs. Several examples of these designs are shown in Figure 30. Starting with the angled dipole element, we extended the concept by introducing additional bends to create zigzag dipoles. In addition to creating greater inductance and capacitance for higher-order filtering and a lower profile, the intention was to create an element with more variability in its orientation. However, none of these bent dipole designs further enhanced the performance stability. 

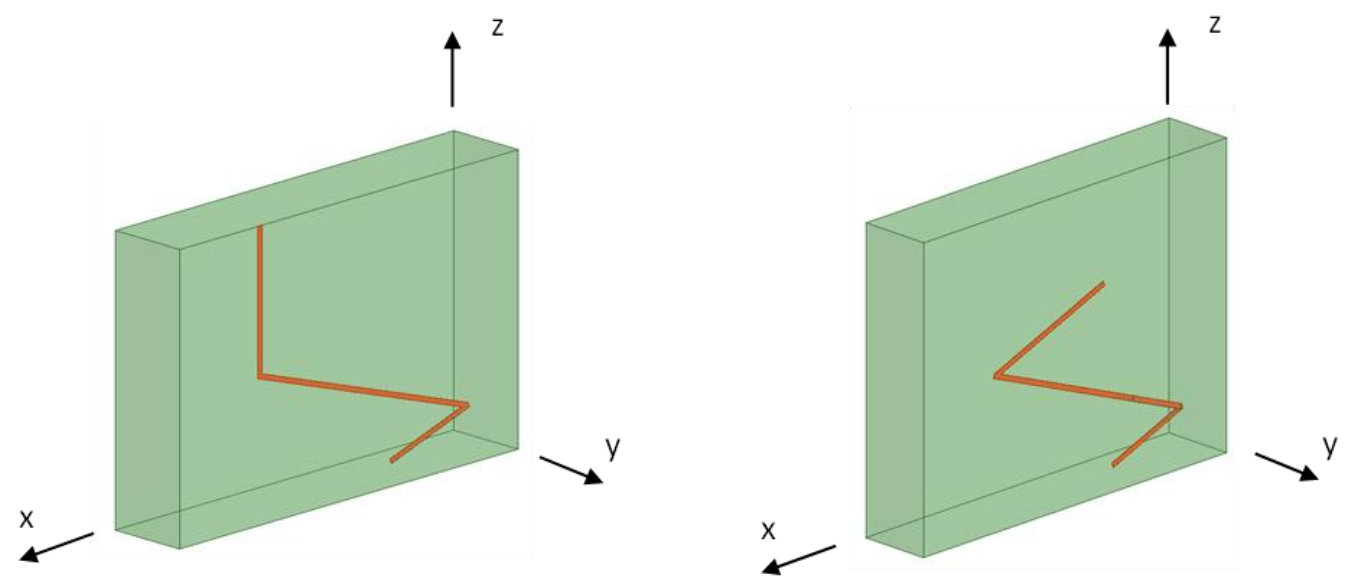

Figure 30. Example dipole elements with additional bends.

\subsection{Curved Element Designs}

From these and other test cases, we deduced that the orientation of the segments in the element plays an important role in the coupling to various incidence angles, and therefore to the performance stability of the design. To this end and to ensure FSS frequency responses, we next considered a different class of periodic elements. The foundation for most of these designs was to utilize an element with curved edges to encourage coupling to a wider variety of incoming waves while maintaining some symmetry within the element design. We therefore transitioned from sharp bends to a design that uses a semi-circle dipole element as shown in Figure 31. In this way, the element has an infinitesimal segment oriented in every possible direction of an incoming wave. In addition to exploring stability, this design provided insight into the design parameters and impact on performance. Also of note, we began simulating designs using 
substrate parameters of $\varepsilon_{\mathrm{r}}=3.50, \tan \delta=0.0015$, in order to replicate Rogers RO3035 commercial printed circuit board (PCB) material in preparation for fabrication.

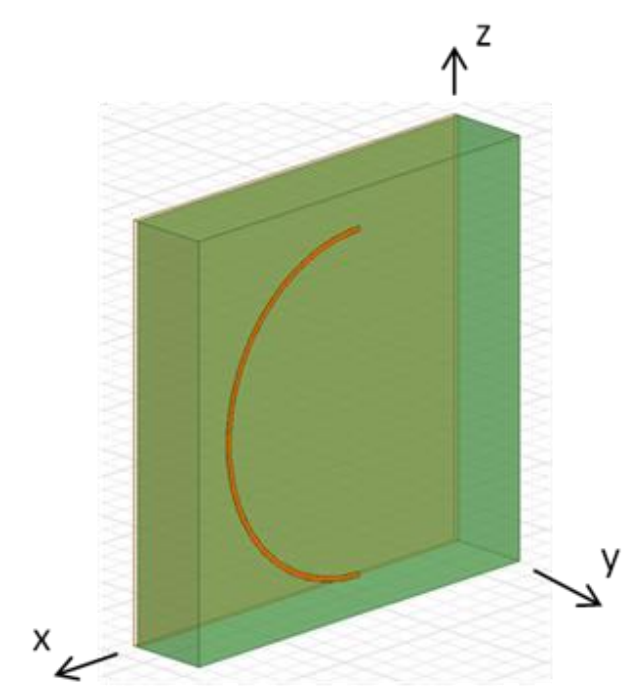

Figure 31. Semi-circle dipole element.

The $S_{21}$ response for this semi-circle dipole element is displayed in Figure 32. This represents the most successful design thus far, with a rejection band that partially covers the same frequency range up through an incidence angle of $40^{\circ}$. We note the tradeoff that exists between selectivity and stability, as the rounded element achieves less selective rejections than the earlier dipoles with sharp bends. However, this tradeoff is worthwhile in order to obtain a design that is stable across a wide range of incidence angles, lending to practical applications. These simulations support the role that element orientation plays in terms of coupling to the incident plane wave. 


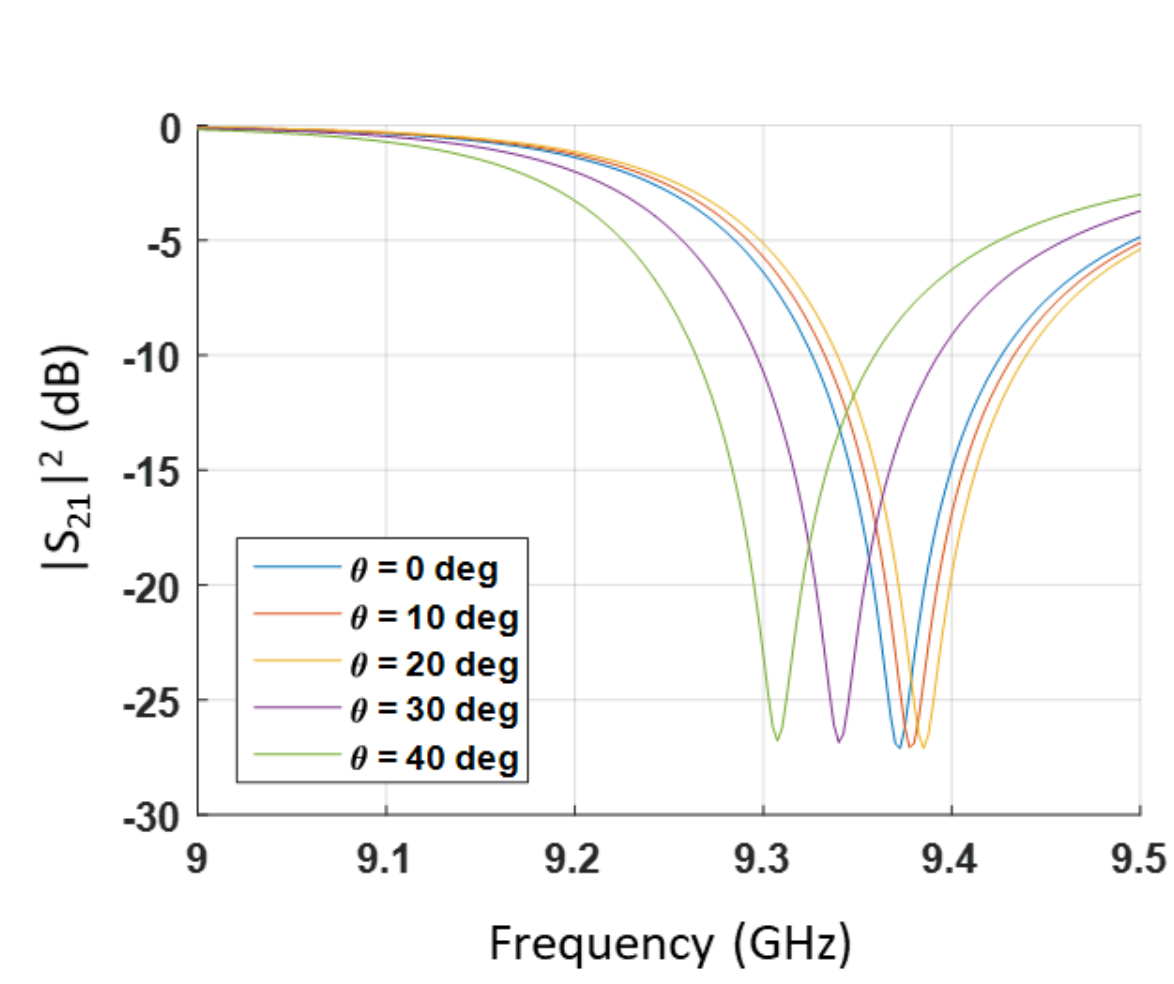

Figure 32. $\mathrm{S}_{21}$ response for semi-circle dipole element, with varying $\theta$.

By creating less abrupt changes in the element geometry, we better accommodate transitions among different incidence angles. To verify this design principle, we developed another geometry solely as a proof of concept. Using the same semi-circle geometry and unit cell dimensions, we simulated a PEC element with a torus shape resulting in rounded-off edges as opposed to squared-off edges. Figure 33 demonstrates this difference by comparing the side views of the two elements, with Figure 33(a) displaying the original semi-circle and Figure 33(b) showing the torus semi-circle element. 


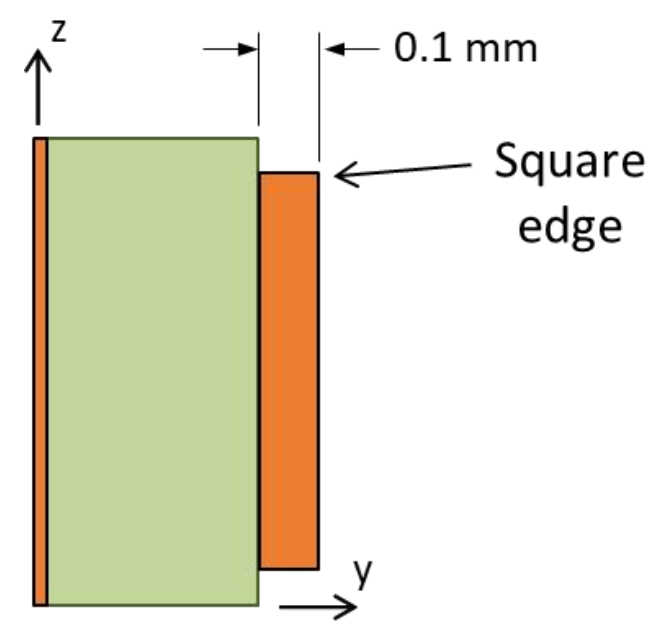

(a)

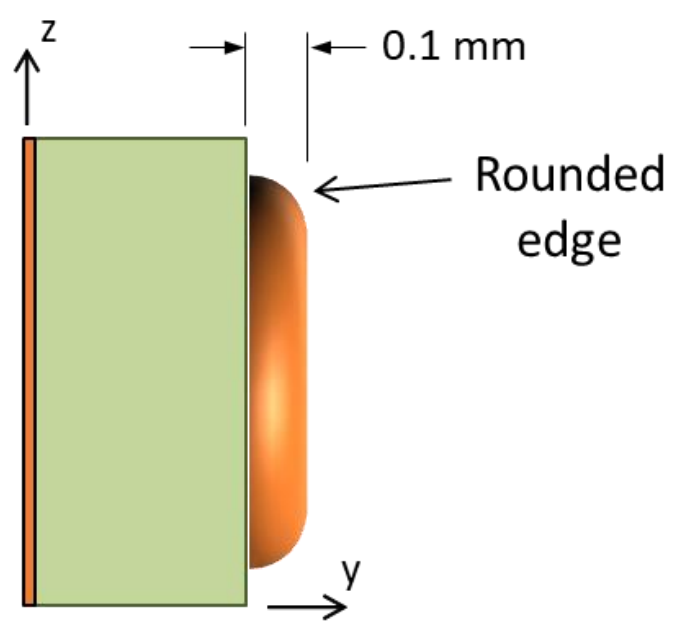

(b)

Figure 33. Side view of semi-circle dipoles: (a) original element and (b) torus element.

For the torus element, the basic geometry is identical to that pictured in Figure 31. However, as shown in the diagram the patch itself is rounded - unlike all previous elements which have a square edge when viewed in the yz-plane. Keeping in line with the design technique of rounding the design to smooth transitions and encourage coupling to a wider range of incoming waves, the torus design takes this concept to the extreme. Note that for both cases shown in Figure 33, the patch depth is exaggerated. Instead of drawing to scale, the sketch is intended to display the difference between these two designs.

Figure 34 displays the $\mathrm{S}_{21}$ performance for this design for varying $\theta$ at $\phi=0^{\circ}$. This torus element exhibits a significant improvement in the stability when compared with any of our previous designs, and the performance is remarkably stable through $\theta=50^{\circ}$. 


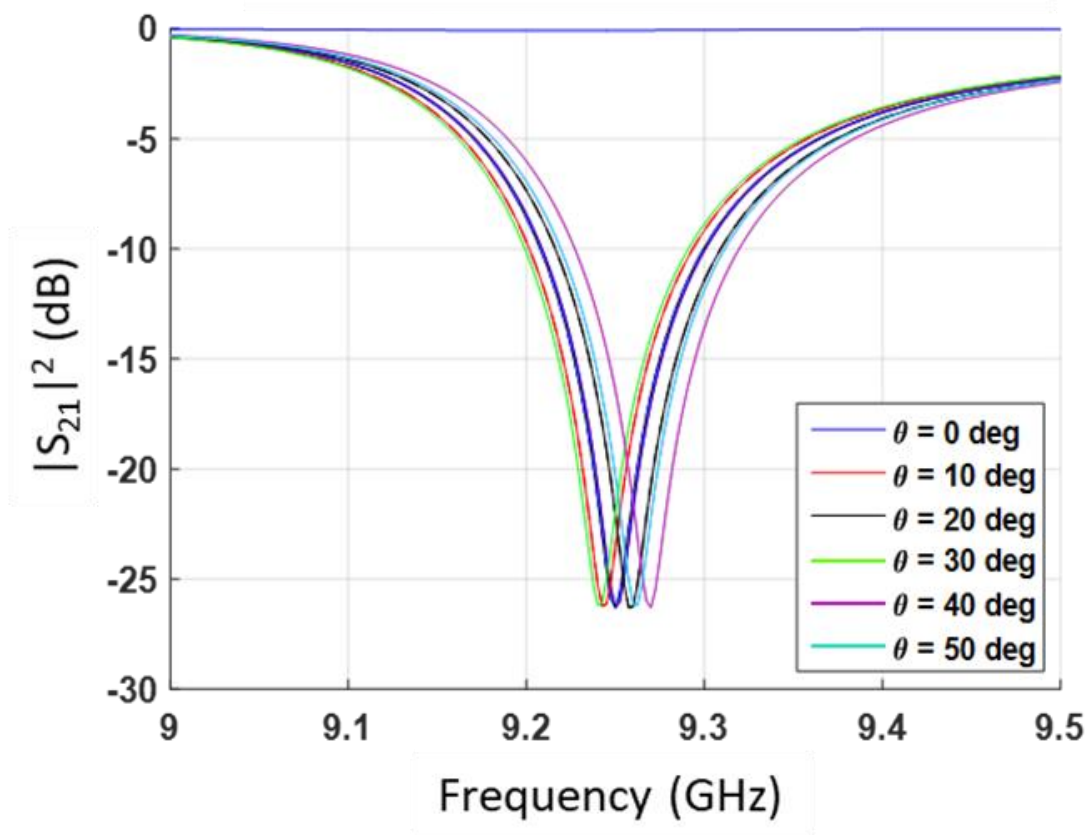

Figure 34. $\mathrm{S}_{21}$ performance for torus semi-circle dipole for varying $\theta$.

While this torus design exhibits exceptional performance stability and better controls the frequency response, a shortcoming is that it is expensive to fabricate. Ideally, a final design would enable use of a commercially available standard double-layer PCB, where one side could be etched to create the element, and one side would remain to create the PEC backing. By utilizing a torus contour for the element, the design pictured in Figure 33(b) would require considerable detail and microfabrication during the manufacturing process instead of simply etching away the element - significantly increasing the cost. Nevertheless, we confirmed our understanding of the value of rounded edges as an important design principle for reconfigurable thick FSS elements. 


\subsection{Circular Elements}

In maintaining focus on rounded edges, we turned to circular element designs, an example of which is shown in Figure 35. This full-circle design was a further attempt to improve stability relative to the semi-circle element.

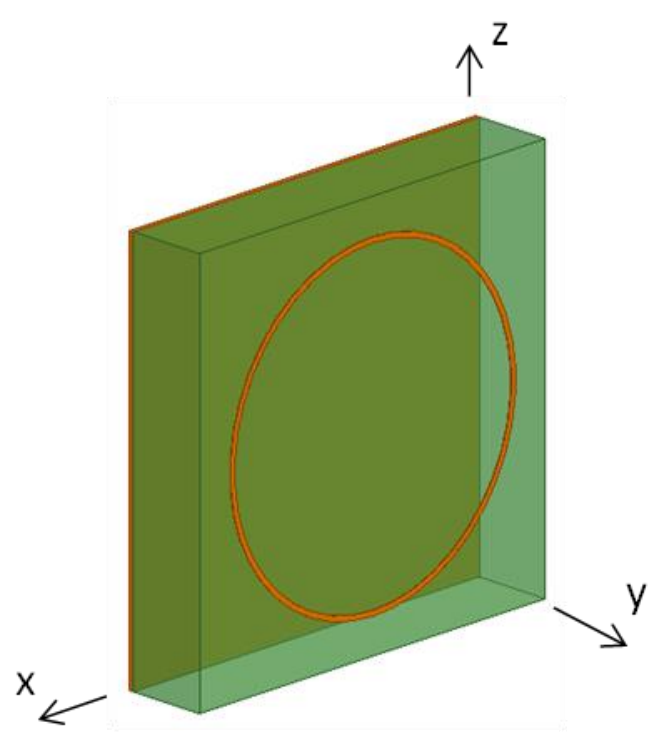

Figure 35. Full-circle dipole element.

By returning to square edges for the PEC element, this is again a design that would be economical to fabricate using the etching process described above. However, the circular element is more limited in terms of reducing the unit cell width. We see the exceptional performance stability exhibited by this design in Figure 36, where there is almost no frequency shift through an incidence angle of $45^{\circ}$. A tradeoff is that this design is less selective, producing a wider relative bandwidth for the filter. There is also an 
additional resonance that appears around $9.7 \mathrm{GHz}$; this is due to coupling, and can be optimized though element spacing and other design parameters used to control the frequency response.

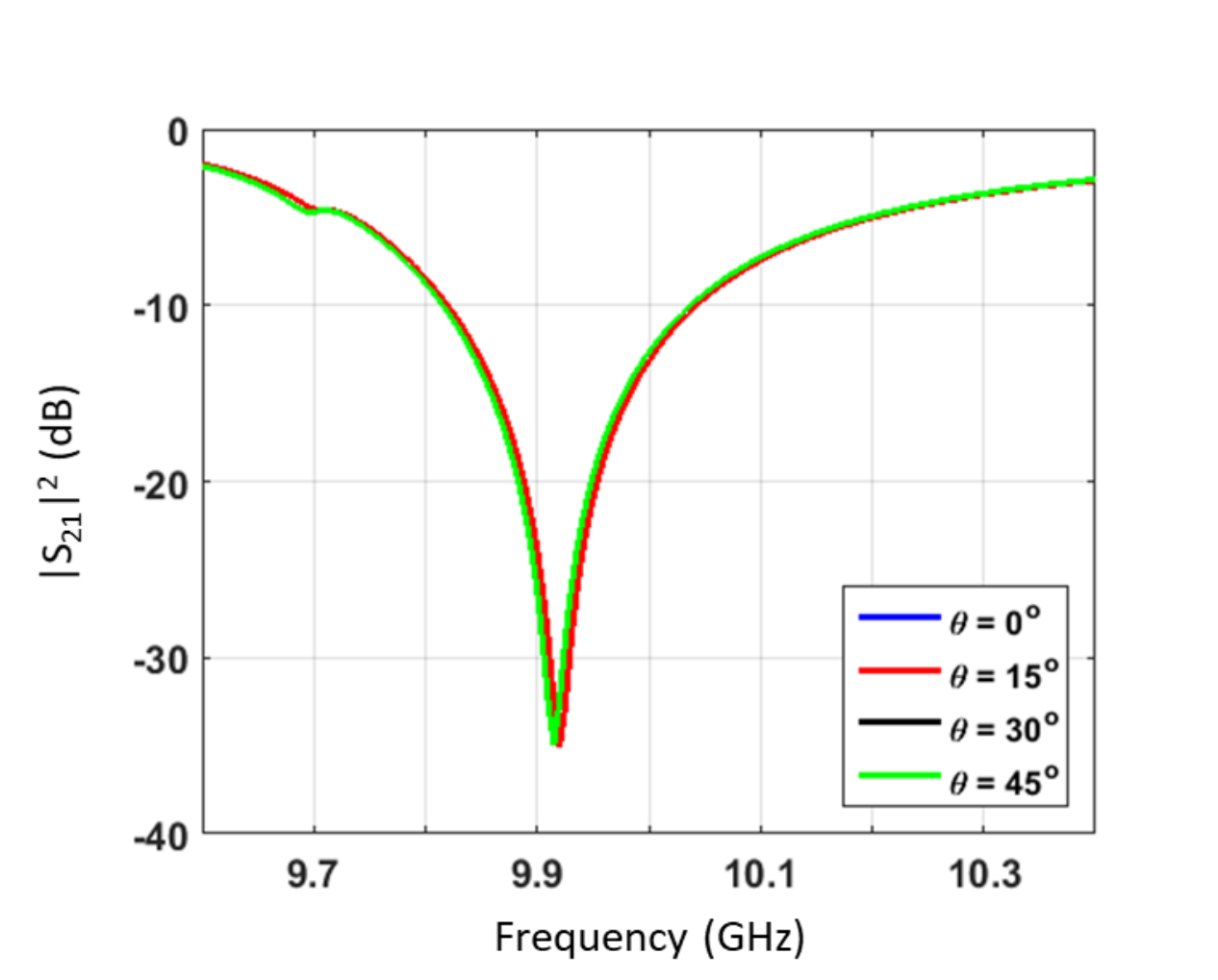

Figure 36. $\mathrm{S}_{21}$ performance for full-circle element, for varying $\theta$.

Since this foundational element demonstrates exceptional performance stability, we again explored methods to implement frequency reconfiguration. We achieved some success with tunable designs, by introducing gaps into the circular element as shown in Figure 37. The $S_{21}$ performance for this element design is displayed in Figure 38. 


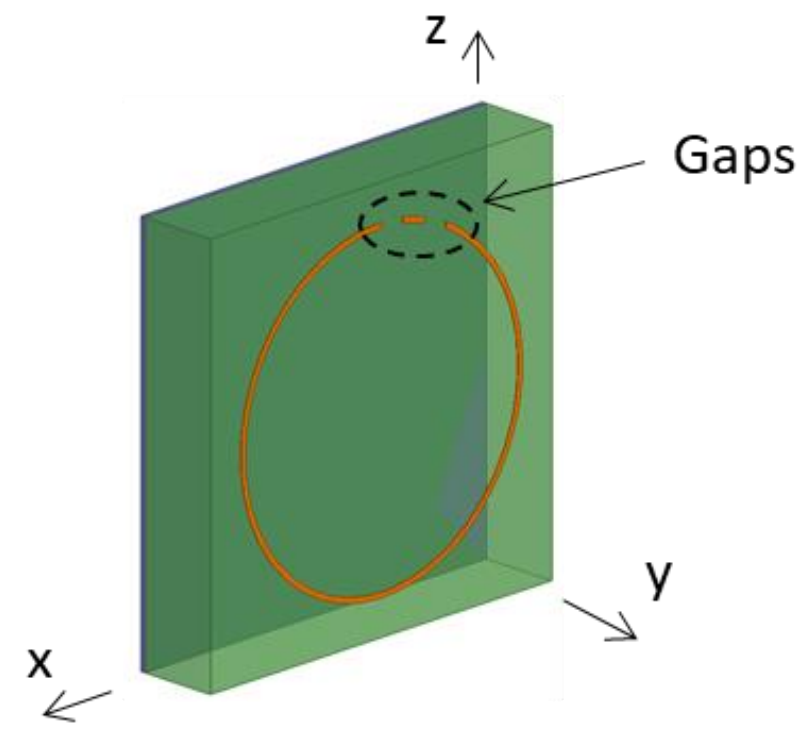

Figure 37. Full-circle dipole element, with gaps for reconfiguration.

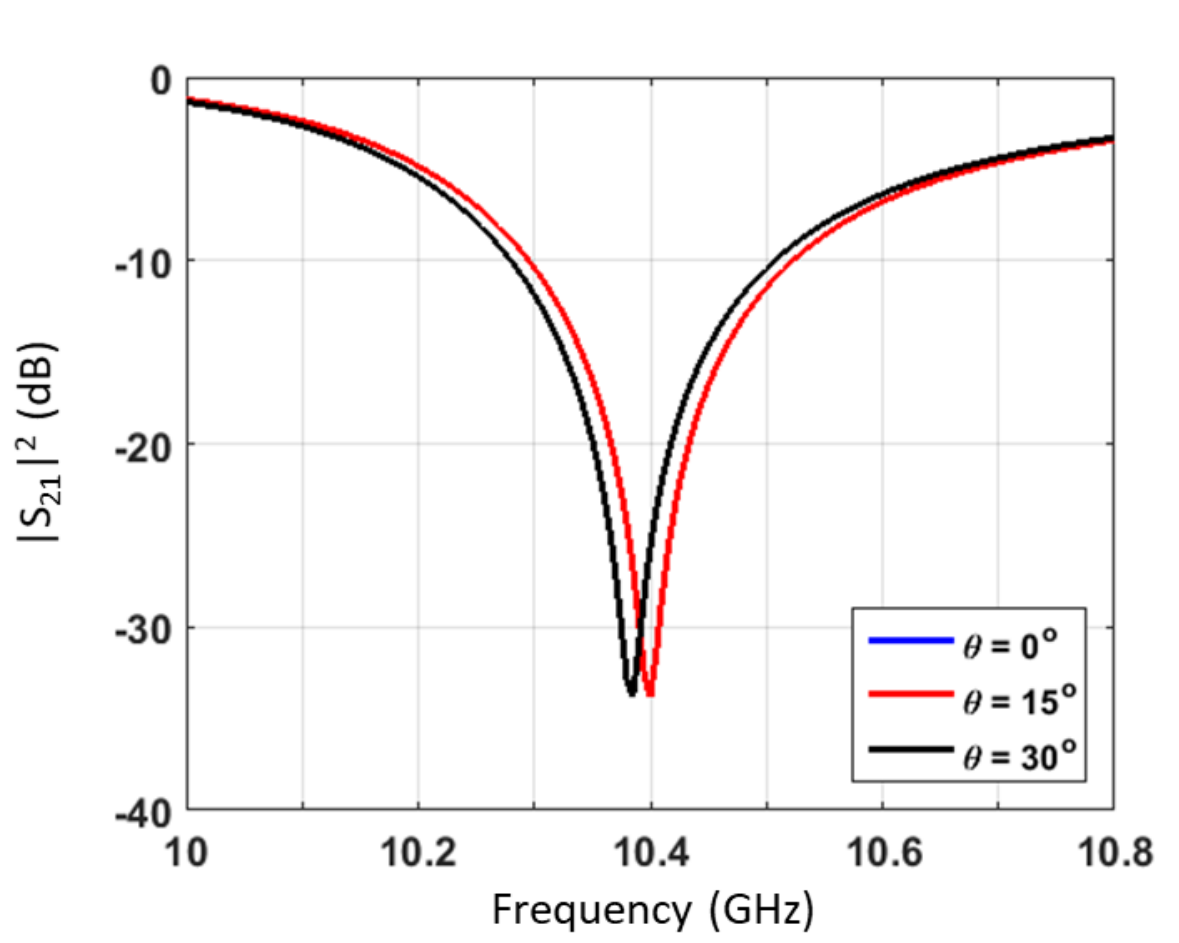

Figure 38. $\mathrm{S}_{21}$ performance for circular element with gaps, for varying $\theta$. 
This reconfigurable design maintained exceptional stability with incidence angle while shifting the rejection frequency from $9.9 \mathrm{GHz}$ to $10.4 \mathrm{GHz}$. However, additional testing for different gap placements only reinforced the benefits of a symmetric design. Further, the shape of the circular element significantly impacts the unit cell size, and the element's width-to-height ratio requires a concession to accept either coupling from adjacent elements or large profile (due to increased element spacing in the x-direction).

\subsection{Reconfigurable FSS Using Elliptical Element}

After exploring many designs (including modified bent dipoles, sinusoids, semicircles, and circular elements), the design that exhibited the best performance is an elliptical FSS element, with its major axis oriented along the direction of propagation (i.e. in the z-direction). This element is depicted in Figure 39. While maintaining the established design principles of rounded edges and symmetry within the element design, the elliptical element also provides a greater projection of the element along the direction of propagation relative to the circular elements. This also ensures a lower profile design, as the unit cells can be more tightly spaced in the x-direction. 

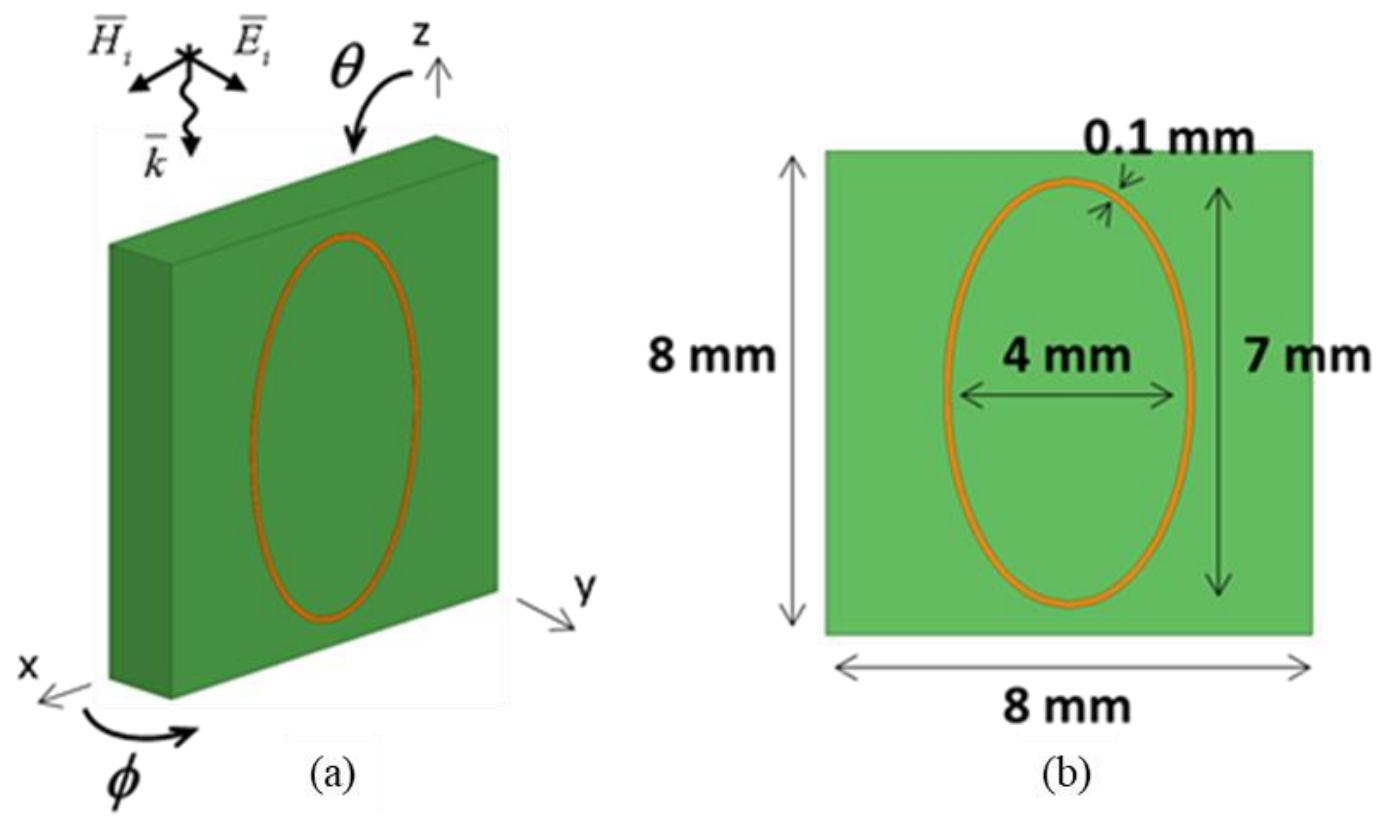

(b)

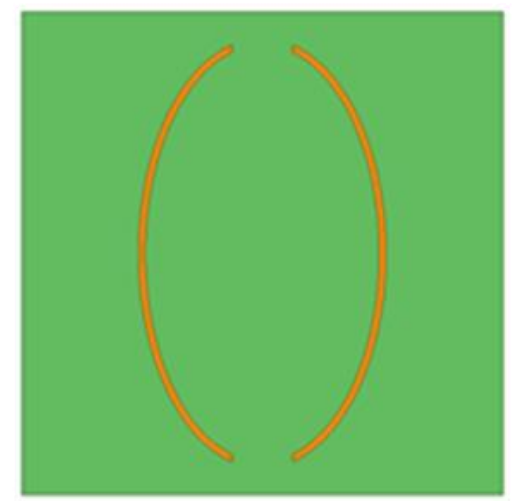

(c)

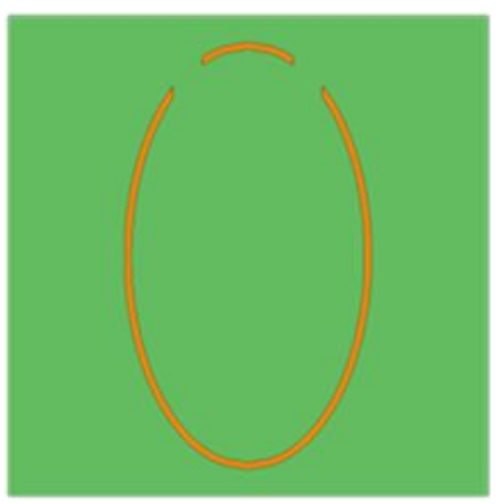

(d)

Figure 39. Elliptical element design: (a) 3-D unit cell, (b) full ellipse, (c) ellipse with gaps at top and bottom of the element, and (d) ellipse with two symmetric gaps near the top of the element.

Figure 39 displays three instances of a reconfigurable elliptical element design. Figure 39(a) provides the 3-D unit cell, with the propagation direction and the electric field orientation. Figure 39(b) displays the base design, with a full ellipse (i.e. no gaps) and dimensions of $7 \mathrm{~mm}$ tall and $4 \mathrm{~mm}$ wide. Figure 39(c) introduces gaps to the top and 
bottom of the element for possible reconfiguration. Finally, for more control for reconfiguration, Figure 39(d) uses two symmetric gaps near the top of the element. All three designs use the same size for the unit cell ( $8 \mathrm{~mm}$ tall and $8 \mathrm{~mm}$ wide) and elliptical element ( $7 \mathrm{~mm}$ tall and $4 \mathrm{~mm}$ wide), and the layer dimensions and material properties are the same as for all the curved FSS element designs.

Figure 40 shows the $S_{21}$ performance for the design pictured in Figure 39(b) - the full ellipse. Clearly this elliptical design exhibits exceptional $S_{21}$ performance stability for varying $\theta$.

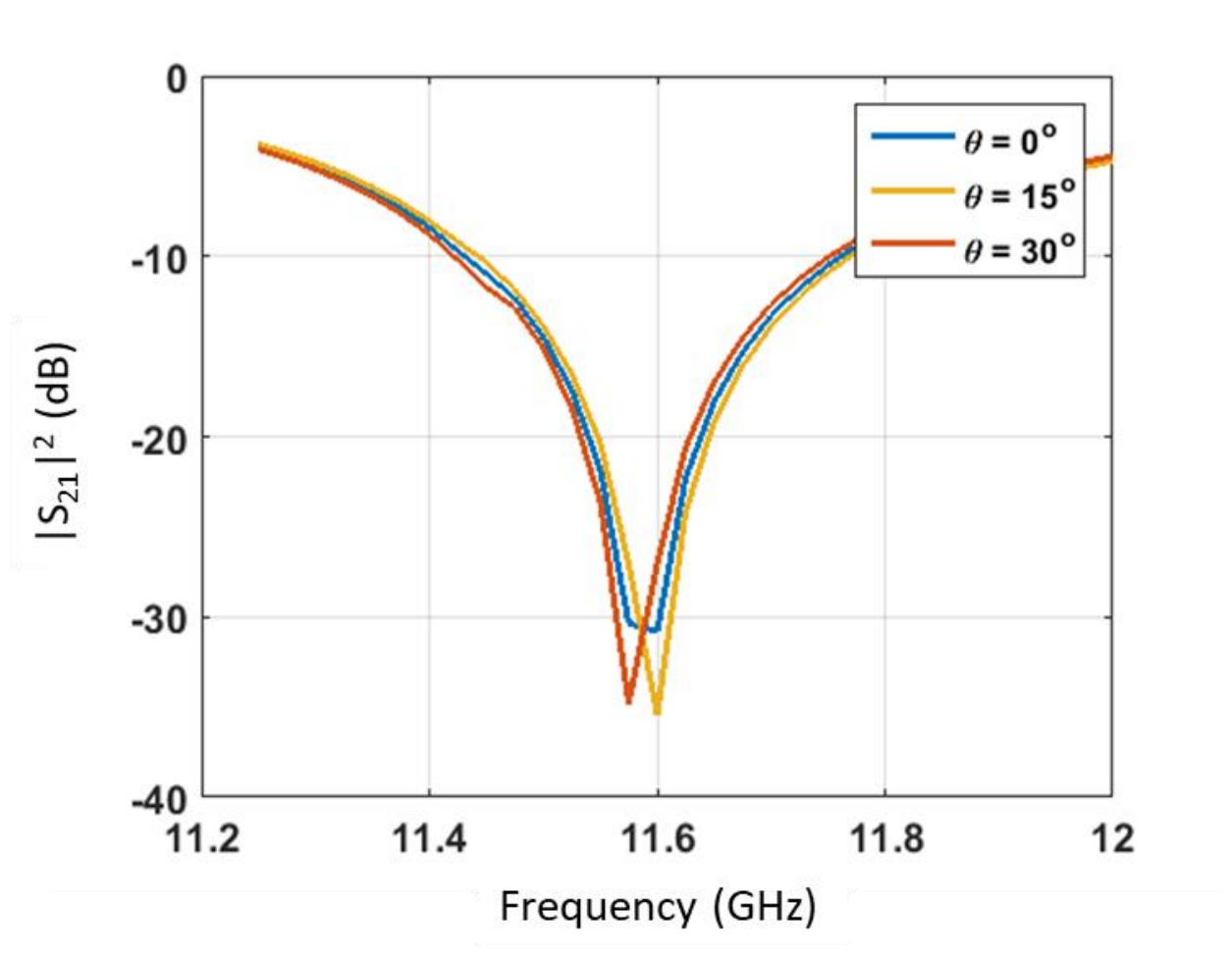

Figure 40. $\mathrm{S}_{21}$ performance for full ellipse, for varying $\theta$. 
Similar comparisons were made for the other two reconfigurable element designs in Figure 39(c) and Figure 39(d), displayed in Figure 41 and Figure 42, respectively. Despite the fact these elements are both incomplete ellipses (i.e. with gaps), the designs continue to demonstrate excellent stability.

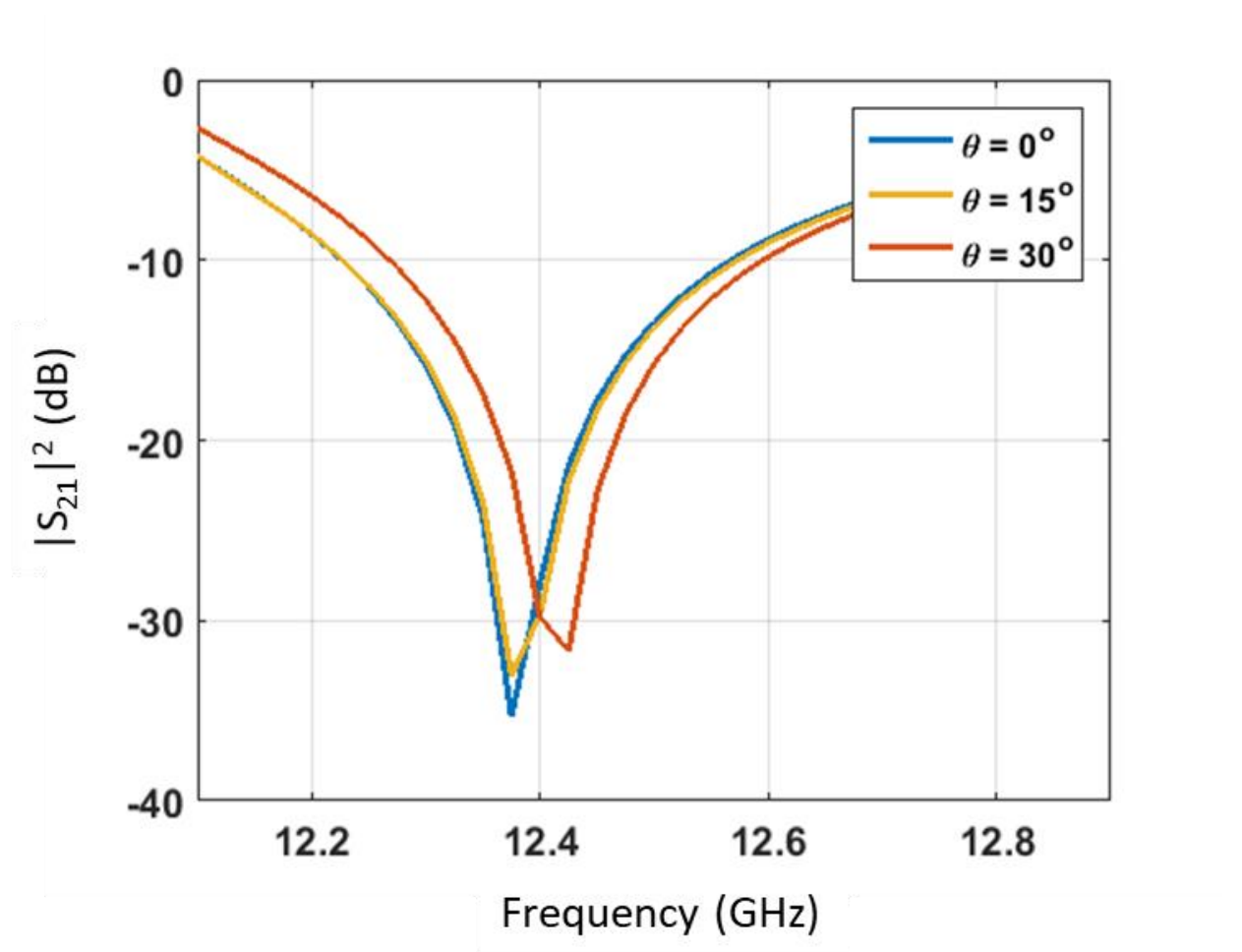

Figure 41. $\mathbf{S}_{21}$ performance for ellipse with gaps at top and bottom, for varying $\theta$. 


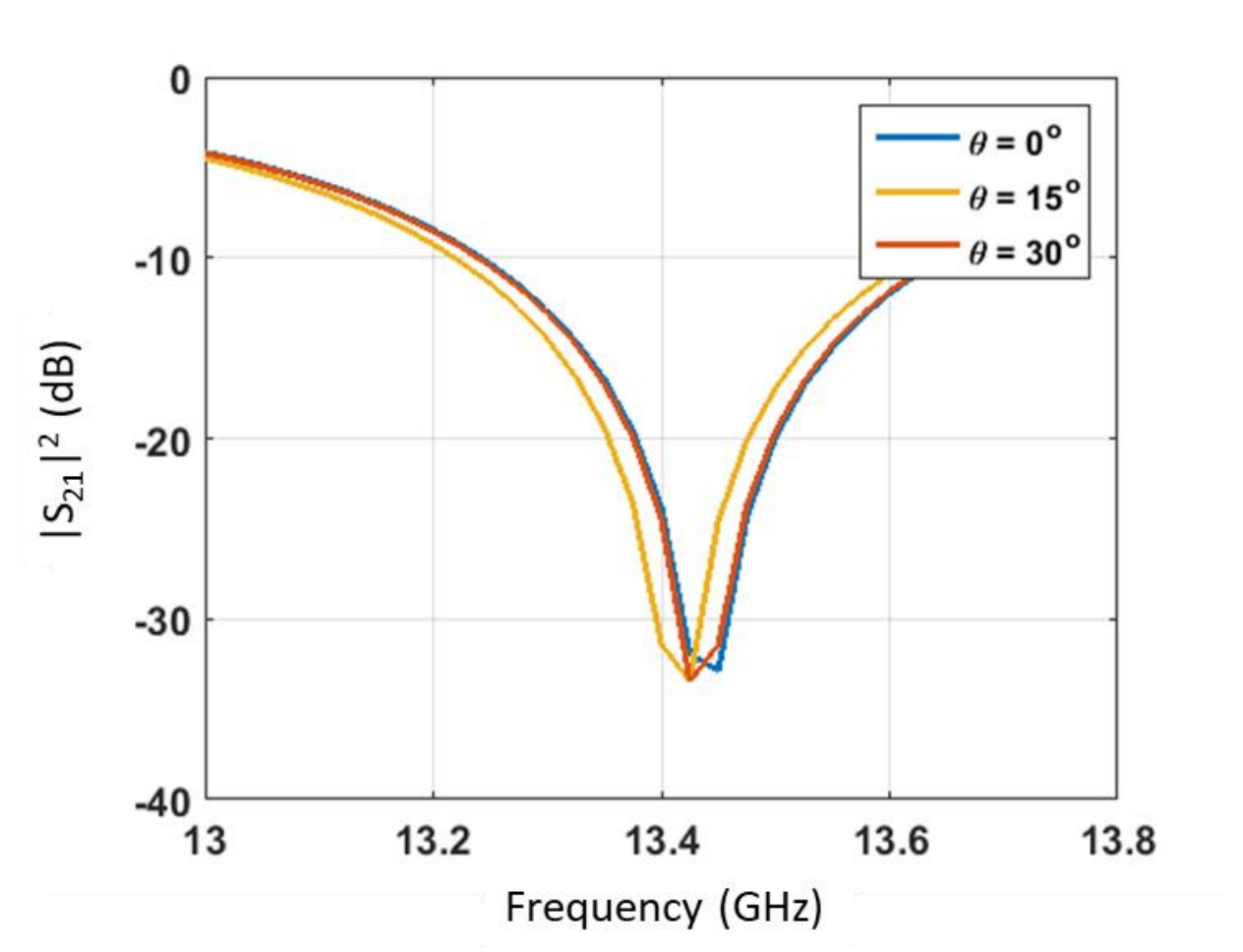

Figure $42 . \mathrm{S}_{21}$ performance for ellipse with two symmetric gaps near top, for varying $\theta$.

All three designs use the same size for the elliptical element and demonstrate performance stability within each design - as well as frequency reconfigurability across the three designs. This frequency reconfiguration is summarized in Figure 43, where we compare the $S_{21}$ performance for each of the three elements at normal incidence $\left(\theta=0^{\circ}\right.$, $\phi=0^{\circ}$ ). From the plot, we see that each of the three designs produces a different rejection frequency, with frequency shifting achieved within the 11-14 GHz range. We note that symmetry is maintained for each reconfiguration of the element design, which contributes to this performance. 

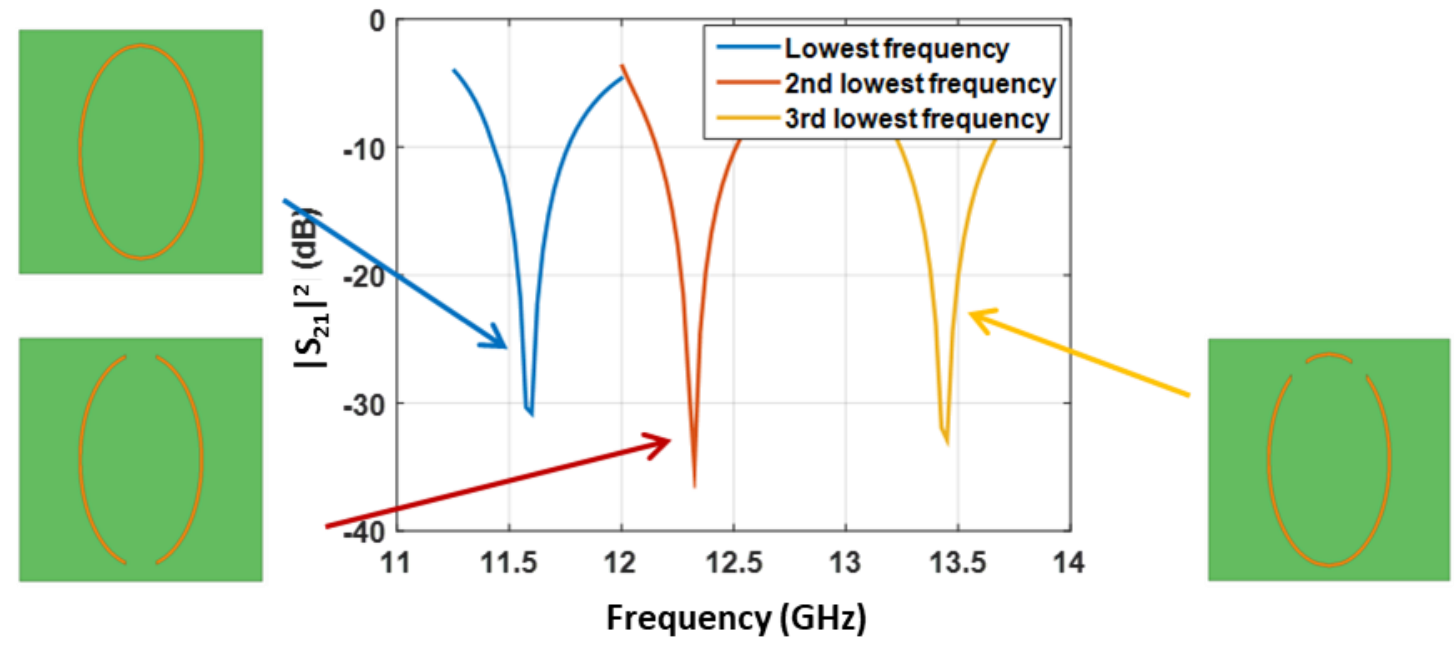

Figure 43. $\mathrm{S}_{21}$ performance for different reconfigurations of elliptical FSS element, with normal incidence angle $\left(\theta=0^{\circ}, \phi=0^{\circ}\right)$.

Since these three designs are all built upon the same fundamental elliptical element, switching would potentially lead to frequency reconfiguration within a single unit cell design. One drawback of using curved element designs is that some selectivity is lost from the earlier bent dipole designs. However, this tradeoff is worth using for achieving the significant performance stability demonstrated by the elliptical FSS element. We can now use this elliptical element as the foundation for fabricating a reconfigurable thick FSS design. 


\section{Chapter 6. Fabrication and Measurements for Validation}

Upon achieving a reconfigurable elliptical design that is stable with respect to incidence angle, we proceeded to fabrication and measurement of prototype designs to validate simulated performance of the FSS elements. We created two separate FSS arrays using the reconfigurable elliptical design, one for the element in Figure 39(c) and one for the element in Figure 39(d). In addition to exhibiting exceptional performance stability, a key advantage of these designs is that they were cost-effective to fabricate since our simulated designs utilize stock sizes of double-layer printed circuit boards (PCBs) that are readily available (based on permittivity, loss, and thickness).

\subsection{Fabrication and Assembly}

We worked with Candor Industries to fabricate these two prototype FSS arrays. A commercially available (off-the-shelf) standard double-layer PCB was used from Rogers Corporation - RO3035 high frequency circuit material. This material is a rigid, thermoset laminate, capable of being processed by automated handling systems and scrubbing equipment used for copper surface preparation. Our simulations were run with 
these material parameters in mind (i.e. $\varepsilon_{\mathrm{r}}=3.50, \tan \delta=0.0015$, dielectric thickness $=$ $1.524 \mathrm{~mm}$, and $1 / 2-\mathrm{oz}$. copper foil).

We designed the finite FSS arrays to be at least $4 \lambda \times 4 \lambda$ in size. This design decision balances approximation of an infinite array (to minimize edge effects) with the practicality of a finite design for cost, fabrication, and measurement considerations. As a result, each fabricated array consists of 17 unit cells across (in the x-direction) to create 1-D periodic strips, and 48 layers of strips (in the y-direction). The strips were created from a large sheet of double-sided PCB material, with one side etched to create the microstrip element while leaving the other side intact to serve as the PEC backing. The 1-D arrays were then cut from the board into shorter (equal length) strips to create the individual layers, with mounting holes drilled for use during assembly. These strips could then be stacked (with air layer separations) to form the FSS and bound at the ends. Standard and well-established circuit board and processing techniques were used for the fabrication, as the boards do not require specialized via preparation processes such as sodium etch.

Figure 44 details the creation of an FSS strip from the unit cell in Figure 39(c) for the fabrication process described above. Figure 44(a) shows a side view of the unit cell, and Figure 44(b) displays the front view of the unit cell with designed dimensions. As demonstrated in Figure 44(c), 17 of these unit cells are repeated periodically to create a single strip. As pictured, each strip was fabricated with two drilled mounting holes (one at each end) for assembly. The same process was used to fabricate the second set of strips from the unit cell design in Figure 39(d). 

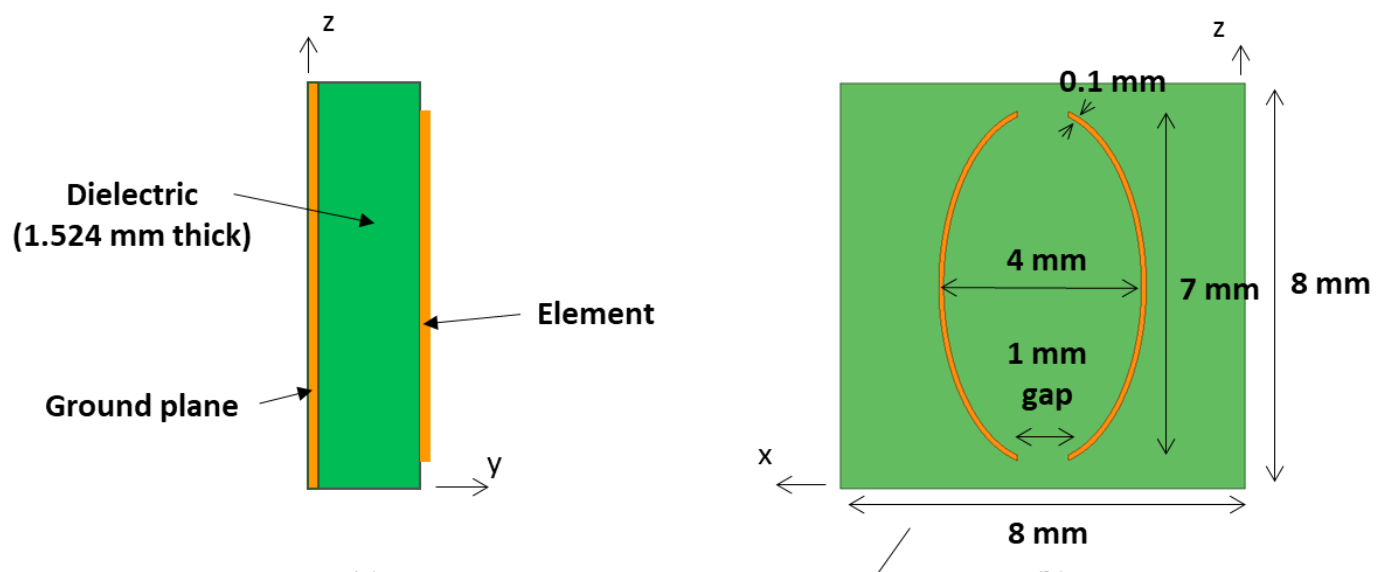

(a)

(b)

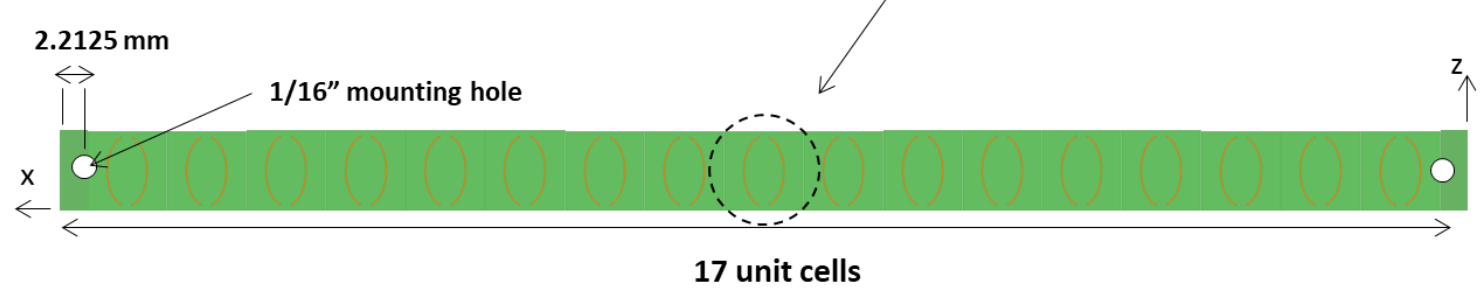

(c)

Figure 44. Creation of strip layers for fabricated designs: (a) side view of unit cell, (b) front view of unit cell with dimensions, and (c) 1-D strip created from unit cell.

Upon receiving the fabricated strips, we still needed to be creative in assembling the FSS arrays. For each array, we stacked the 48 strips and kept them in place using two $1 / 16$ " diameter plastic rods, with one rod guided through the drilled mounting holes on each side of the strip. To maintain the $1 \mathrm{~mm}$ air gap between layers, we used $1 \mathrm{~mm}$-thick nylon spacers along the plastic rods - spaced between successive strips. This ensured the precise air separation required for our design. Finally, we used small rubber bands to bind the design together at each end of both plastic rods, to maintain the integrity of the overall size and shape of the FSS array. The overall size of each FSS array is 
approximately $5.5 \% \times 5.5 \%$ A photo of one of the assembled arrays is shown in Figure 45(a), with a close-up photo of the unit cell displayed in Figure 45(b).

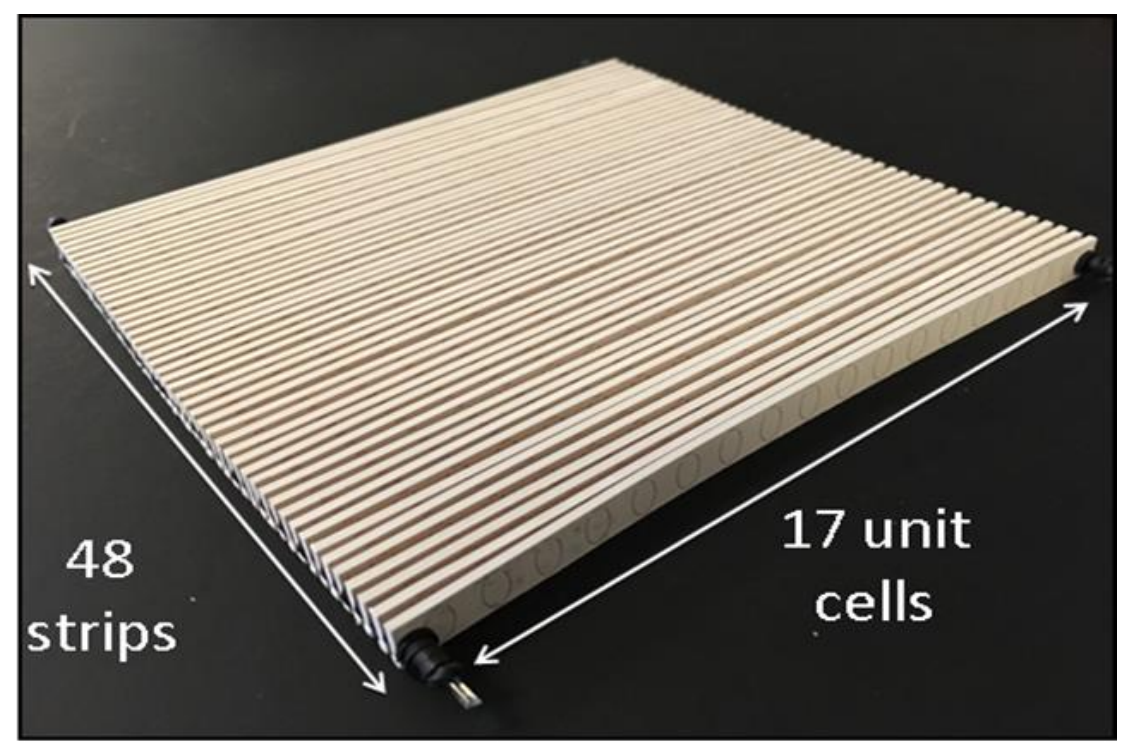

(a)

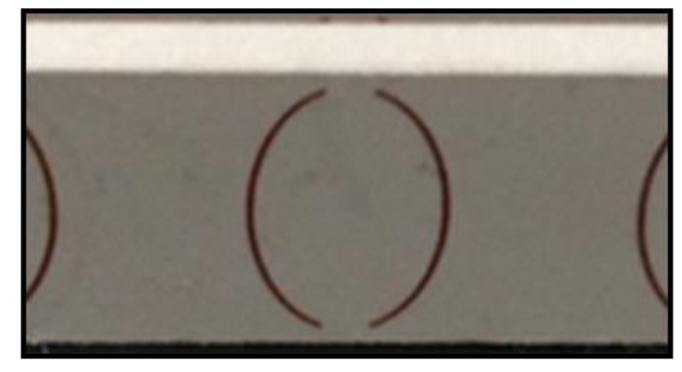

(b)

Figure 45. Fabricated and assembled FSS array: (a) full 2-D array and (b) unit cell. 


\subsection{Measurements}

Once the 2 FSS arrays were assembled, we proceeded with measurements. The measurements were conducted in an open lab environment, using two horn antennas and a network analyzer set up as pictured in Figure 46(a). A close-up photo of the FSS array with one of the antennas is displayed in Figure 46(b).

For the measurement set-up, two identical horn antennas were used for transmit and receive, with a test frequency range from 2-18 GHz. The measurements were conducted at a distance slightly greater than 3 meters, and the antennas and FSS array were mounted on foam pedestals to maintain an equal height through all the measurements. Additionally, we created a foam support to stabilize the FSS array orientation and positioning relative to one of the horn antennas (see Figure 46(b)).

As with simulations, our focus was on transmission, viz. $\mathrm{S}_{21}$ measurements. We conducted measurements at normal incidence (as pictured in Figure 46(a)), as well as at incidence angles of $15^{\circ}$ and $30^{\circ}$. For each incidence angle, calibration was first carried out using an "empty" foam, i.e. the exact same set-up except without the FSS array. Each measurement was then adjusted by its respective calibration test, and these measurements are shown in Figure 47. As displayed, the measurements clearly verify and validate the simulated designs for incidence angles from $0^{\circ}$ to $30^{\circ}$. 


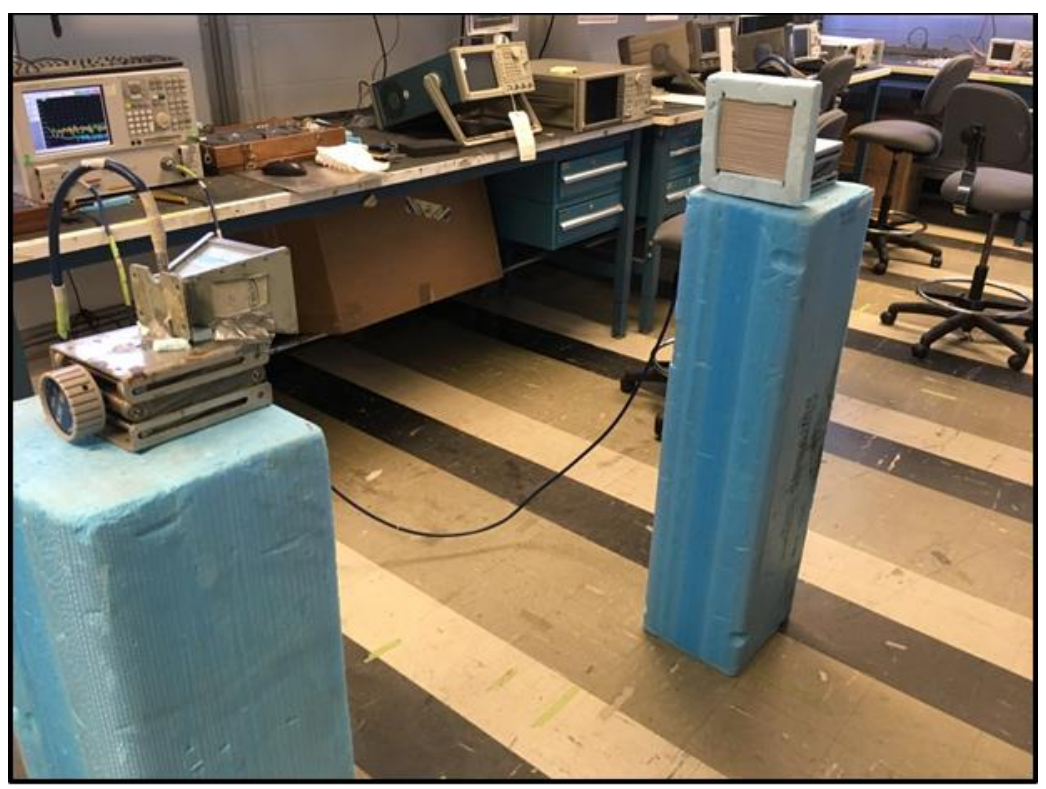

(a)

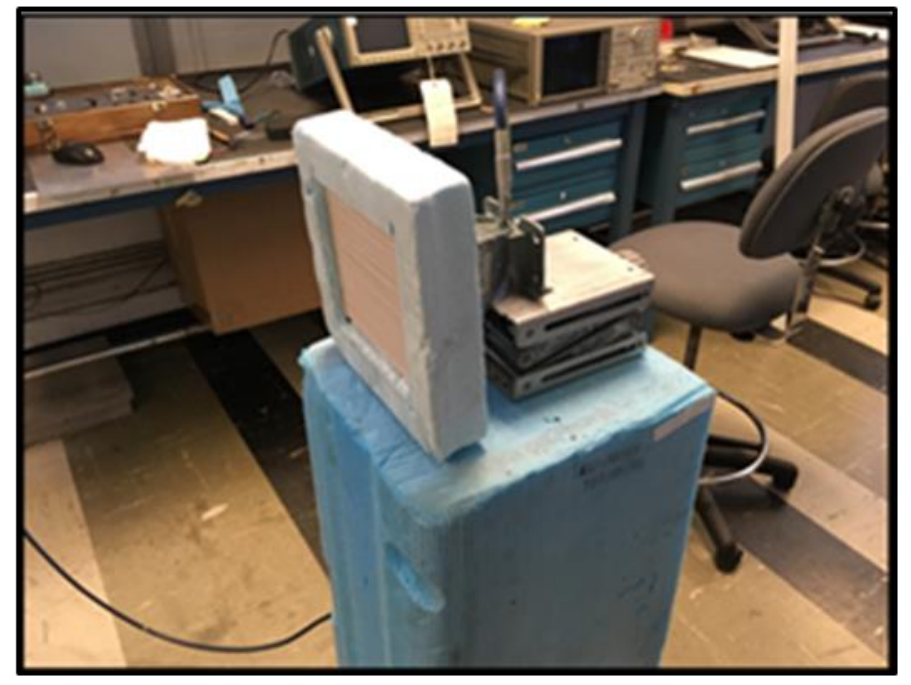

(b)

Figure 46. (a) Measurement set-up and (b) zoomed-in photo of FSS array/antenna configuration. 


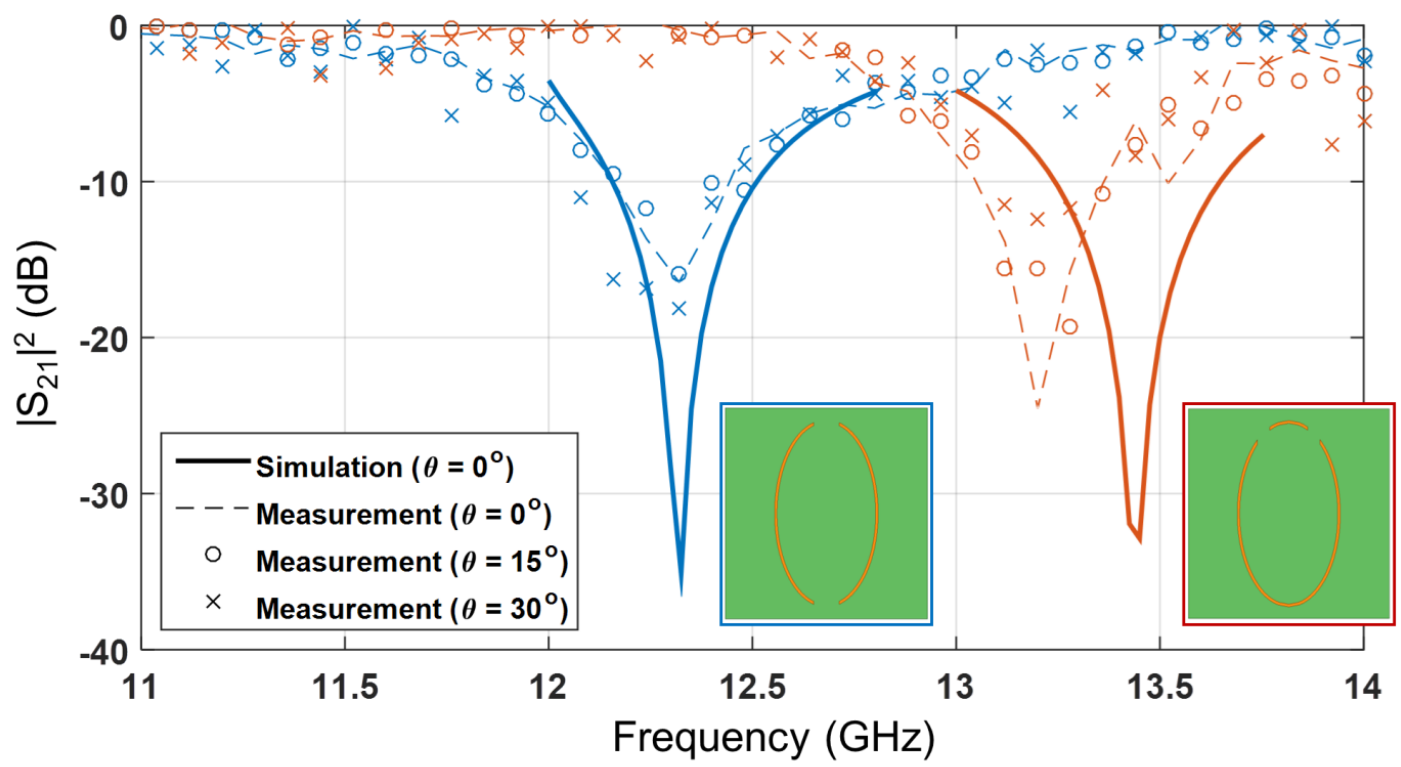

Figure 47. Measurement results for two fabricated FSS arrays, compared with simulations.

As expected, the two different designs successfully achieved frequency reconfiguration using the same foundational elliptical element by altering the placement of the gaps. Additionally, there is good agreement between measurements and simulations in terms of rejection frequency. For the lower frequency design, the frequency match is very good. However, for the higher frequency design there is a $0.25 \mathrm{GHz}$ frequency shift. This shift is believed to be due to change in dimensions during the assembly of the FSS. Since this was intended to be an inexpensive proof-of-concept fabrication, there is some acceptable margin for error in the design. Looking at Figure 45(a), we see some bowing of the strips as the gap size shrinks for several of the air gaps toward the center of the strip.

Nevertheless, the measurements confirm the performance stability of both designs with respect to incidence angle. For both FSS array designs, the measured transmission 
response is stable from normal incidence through $30^{\circ}$. This is a significant achievement, as performance stability was an important objective during the design process particularly for narrowband designs.

We provided a path forward to a new class of tunable thick FSS structures that provide for high flexibility and frequency control across a large bandwidth range. We showed that sharp filter roll-offs were achieved using designs that are readily realizable in practice. By fabricating and measuring prototypes of a stable multi-band design, we have demonstrated a capability to produce multiple rejections in the $11-14 \mathrm{GHz}$ range, with each design tuned to reject a different narrowband frequency. 


\section{Chapter 7. Summary and Future Work}

This work introduced a novel frequency reconfiguration technique using thick FSS-based transmission filters. We provided a path forward to a new class of tunable thick FSSs that provide high flexibility in tunability range, sharp filter roll-offs, and designs that are readily realizable in practice. These thick FSSs demonstrate an ability to control and adjust narrowband rejection frequencies using a single multi-function design, achieving reconfiguration across a wide bandwidth.

Importantly, the proposed designs achieve considerable stability with varying incidence angles, while maintaining a high level of selectivity and a relatively low profile. This performance required the examination of several FSS elements, and we ultimately achieved exceptional performance by focusing on elliptical-shaped designs due to their rounded edges and symmetry. Two different reconfigurations of these elliptical element designs were optimized for fabrication, including cost-effectiveness to be manufactured from off-the-shelf PCBs. These fabricated prototypes were then measured, and the measurements validated the simulated results. These designs exhibit selective rejections that are reconfigurable in the $11-14 \mathrm{GHz}$ frequency range, representing a new and useful direction for tunable filters. 


\subsection{Future Work}

Having developed a solid and innovative design foundation for these tunable thick FSSs, there are several useful extensions of the research that would build on these concepts. In terms of the FSS designs themselves, further work can be done for design and optimization of the geometry shape, size, and orientation to expand the frequency range of reconfiguration and control. While initial designs were produced to exhibit the improved features and performance characteristics, additional configurations can be designed and analyzed for specific frequency ranges for desired applications. Additional design arrangements can also be explored to create an even more compact design, one that minimizes $\mathrm{SWaP}$ requirements for practical and cost-effective applications. The research can also further extend to band-pass capability to enable total control of the operating frequency for one's own systems.

Another extension of this research is to develop designs that integrate actual switching devices for dynamic frequency reconfiguration and control. These designs could be simulated, analyzed, and optimized for switch placement to yield efficient and cost-effective structures. A sample design might then be fabricated as a prototype to test a reconfigurable, switch-based device, thus serving as a proof of concept. These switching devices could potentially incorporate several different approaches for reconfigurations of the operational band, including RF MEMS switching technology (e.g., as pictured in Figure 48) for a reliable and stable approach [85, 86]. 


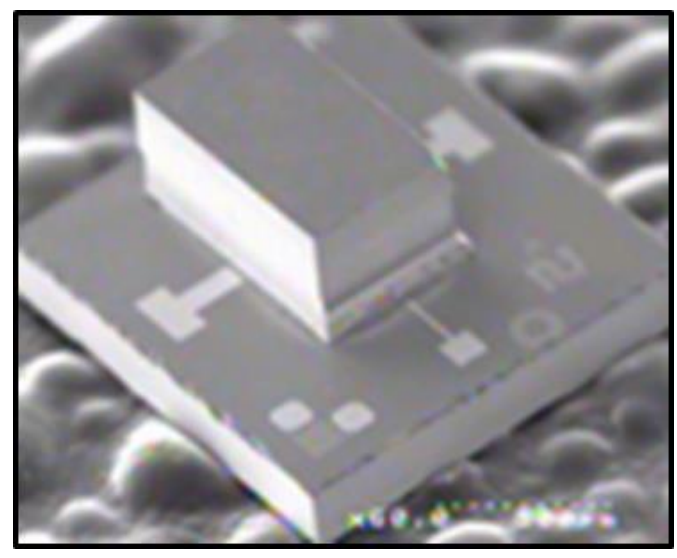

Figure 48. Example RF MEMS switch.

In exploring switches and additional means for reconfiguration, one possibility is to consider three-way switches. By integrating these devices, a design can better minimize the number of active elements in the final structure. One example design that utilizes this three-way switching concept is shown in Figure 49.

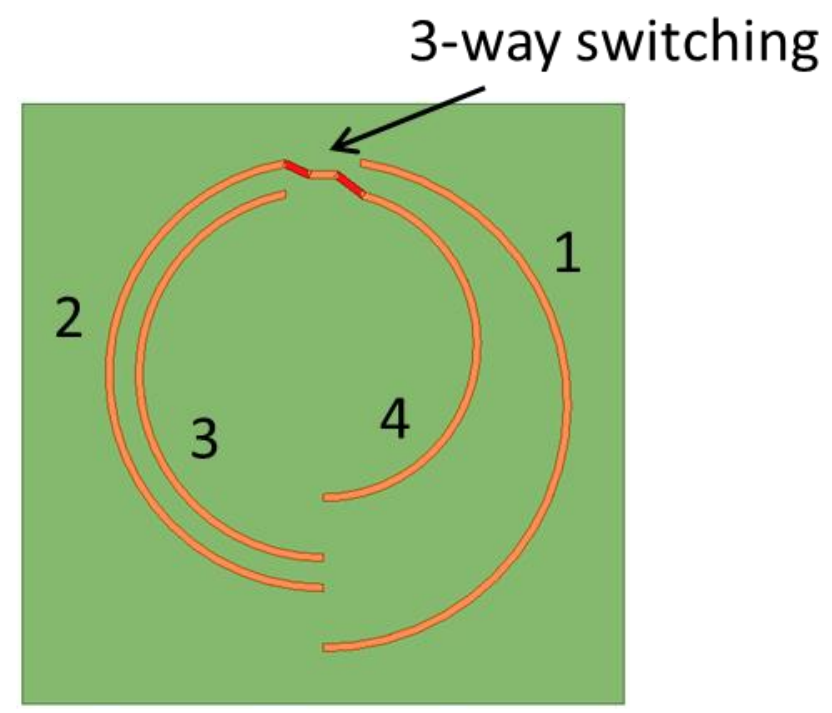

Figure 49. Circular-based element for reconfiguration. 
This design incorporates the circular element concept by creating 4 semi-circles of different sizes. They are numbered here from longest to shortest (i.e. 1 to 4 ). By mimicking the use of two 3-way switching devices (displayed in red for emphasis), different combinations of semi-circles can be connected to create elements of different lengths. This design produces four different combinations to explore reconfiguration across a wide bandwidth, with ' $1-2$ ' being the longest and ' $3-4$ ' being the shortest. As pictured, the switches are positioned to create the '2-4' element.

Figure 50 demonstrates the $S_{21}$ performance for this design configuration, for the third-longest (i.e. '2-4') element. The results are compared for varying $\theta$ at $\phi=0^{\circ}$. Despite the fact that the element is both an incomplete circle and has discontinuities at the switching locations, the design continues to exhibit exceptional performance stability for this element reconfiguration. We do see multiple resonances in the $8-16 \mathrm{GHz}$ frequency range due to the lengths of the parasitic elements, but as long as they are controllable then multiple resonances are not necessarily a problem depending on the application or desired frequency range. While this element represents just one example for further research into reconfigurability, the design shows considerable promise and can similarly be extended to an elliptical element. 


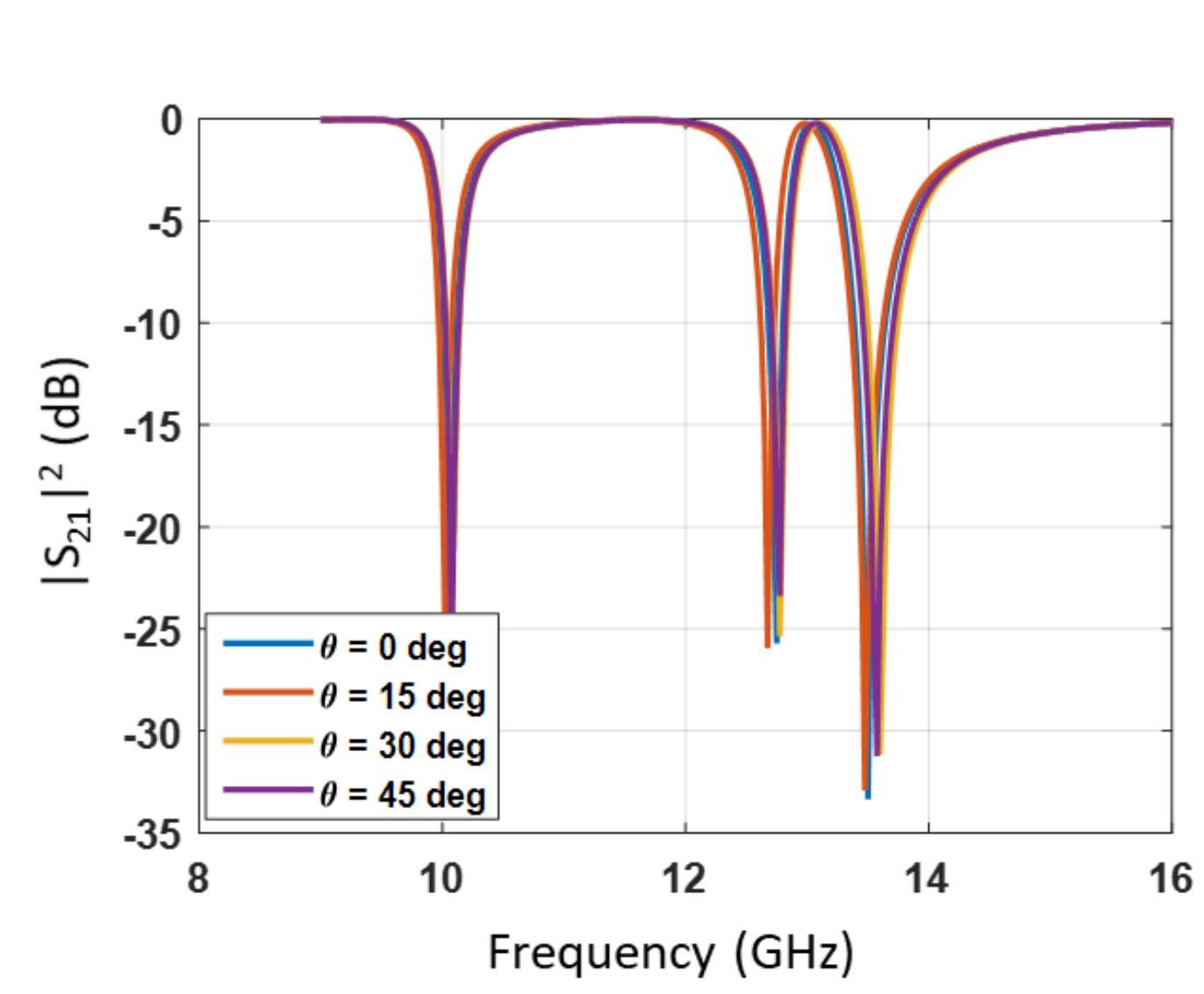

Figure 50. $\mathrm{S}_{21}$ performance for reconfigurable circular element for varying $\theta$.

Another category of future work for this research is to explore the use of these FSS designs as curved (rather than straight) structures to better model real-world systems and environments. The conformal nature of these structures readily enables these arrays to be flexible and adaptable for such applications. Simulating curved FSS designs presents several challenges, as key features are lost from the "planar" thick designs such as periodicity and infinite extent. Without this periodicity, analysis of a curved FSS design cannot be simplified to a single unit cell. Rather, it becomes necessary to perform the computations for groups of elements or individual elements conformed to the 
structure's profile. At times, the entire FSS may even need to be analyzed at once, resulting in increased demands on computational time and memory during the design process.

Due to the complexity and the size of the simulations required to analyze conformal FSS arrays, some numerical acceleration techniques have been pursued. For instance, one method divides a curved FSS into overlapping sub-arrays that are analyzed individually using a rigorous full-wave method [87]. Further, the thick FSS element designs introduced in this research have great potential to be useful for curved surfaces, as they benefit from a sub-wavelength unit cell that is less sensitive to incidence angle [88]. Nevertheless, thick FSS arrays still present a challenge for simulation and design. Therefore, additional research might include numerical modeling and analysis of thick FSS designs applied to curved surfaces, to explore the impact of these real-world limitations on design considerations and results.

\subsection{Possible Applications}

The proposed and experimentally validated reconfigurable thick FSS designs will provide for a new direction of tunable radomes and other applications. For several decades, thin surfaces of planar dipoles, loops, or Jerusalem crosses served as the hallmarks of FSS structures. However, these planar frequency selective surfaces provide minimal selectivity, limiting their value for tunability and design flexibility. 
By contrast, the thick FSS concept elevates these designs to a new class of applications. Similar to how patch antennas adapted from their initial introduction into thick microstrip patch designs for a new direction in conformal wideband antenna design, a parallel comparison can potentially be made between planar and thick FSSs. Just as this evolution made patch antennas and arrays ideal for many commercial and government applications, we believe that thick FSSs will do the same.

By introducing improved selectivity, sharp roll-offs, lower profile, and frequency reconfiguration and control to these thick FSS designs, this dissertation addresses several areas of interest in antenna design for multi-functionality and spectrum management. Potential applications include using these thick FSS designs: (1) as reconfigurable cover layers (e.g., radomes) over broadband antennas; (2) in frequency switching for communication/signal security; (3) to realize UWB operation using narrow, frequencyagile band selections; and (4) for isolation and interference reduction for UWB arrays. Since these designs can be modified to produce either band rejections (e.g., to reject noise jamming) or band-pass behavior (e.g., to enable a protected antenna to dynamically switch operation frequency to avoid being detected and/or jammed), an incredibly diverse set of applications is possible. As such, these structures can enable dynamic adaptation to a changing environment.

Applying tunable thick FSS designs to these applications will require further system integration and testing, including sensor resource management to ensure successful integration of the design into an end system - specifically its integration with the antenna and back-end components. Combined with digital beamforming capabilities, 
such systems will provide for unprecedented capability in terms of agile sensing and communication functions. As a result, the achievements made from this foundational research on reconfigurable thick FSSs supports radomes, apertures, and other critical systems that are needed for future Air Force platforms.

The designs presented in this dissertation demonstrate significant improvements to designs using thick FSSs. By addressing challenges associated with selectivity, tunability, and stability of these structures, we have opened the door for additional innovation in this emerging area. We introduced new elements and design techniques for reconfigurable FSS filters, and this research should lead to further developments in spectrum management and frequency agility. 


\section{Bibliography}

[1] M. Nowak, "Layered sensing to enhance situational awareness," in 2008 International Symposium on Collaborative Technologies and Systems, June 2008.

[2] AFRL/RYM - Multispectral Sensing \& Detection Division. (2016, July 14). https://www.wpafb.af.mil/Welcome/Fact-Sheets/Display/Article/837716/

[3] G. Sklivanitis, A. Gannon, K. Tountas, D. Pados, S. Batalma, S. Reichhart, M. Medley, N. Thawdar, U. Lee, J. Matyjas, S. Pudlewski, A. Drozd, A. Amanna, F. Latus, Z. Goldsmith, and D. Diaz, "Airborne Cognitive Networking: Design, Development, and Deployment," IEEE Access, vol. 6, pp. 47217-47239, July 2018.

[4] E. Y. Imana, "Cognitive RF front-end control," Ph.D. dissertation, Virginia Polytechnic Institute and State University, 2014.

[5] B. A. Munk, Frequency Selective Surfaces: Theory and Design. New York, NY, Wiley, 2000.

[6] T. K. Wu, Frequency Selective Surfaces and Grid Arrays. New York, NY, Wiley, 1995.

[7] S. B. Glybovski, S. A. Tretyakov, P. A. Belov, Y. S. Kivshar, and C. R. Simovski, "Metasurfaces: From microwaves to visible," Physics Reports, vol. 634, pp. 1-72, May 2016.

[8] J.C. Vardaxoglou, Frequency Selective Surfaces: Analysis and Design. Boston, MA, Research Studies Press, 1997.

[9] H.-H. Sung, "Frequency Selective Wallpaper fot Mitigating Indoor Wireless Interference," Ph.D. dissertation, The University of Auckland, New Zealand, 2006.

[10] S.-W. Lee, "Scattering by dielectric-loaded screen," IEEE Transactions on Antennas and Propagation, vol. 19, pp. 656-665, Sept. 1971. 
[11] E. Pelton and B. Munk, "A streamlined metallic radome," IEEE Transactions on Antennas and Propagation, vol. 22, pp. 799-803, Nov. 1974.

[12] S. Monni, G. Gerini, and A. Neto, "Frequency selective surfaces for the RCS reduction of low frequency antennas," in Proc. EuCAP 2006, Nov. 2006.

[13] P. Wang, P. Tang, W. Luo, Z. He, L. Li, and S. He, "Design of dual-band frequency selective surface for antenna RCS reduction," in 2016 Progress In Electromagnetic Research Symposium, pp. 4116-4119, Aug. 2016.

[14] S. Agahi and R. Mittra, "Design of a cascaded frequency selective surface as a dichroic subreflector," in Proc. Antennas and Propagation Society International Symposium: AP-S Merging Technologies for the 90 (Digest), pp. 88-91, May 1990.

[15] J. Arnaud and F. Pelow, "Resonant-grid quasi-optical diplexers," Bell System Technical Journal, vol. 54, pp. 263-283, Feb. 1975.

[16] X. C. Tong, Functional Metamaterials and Metadevices. Springer International Publishing, 2018.

[17] Department of Defense (DoD) Strategic Spectrum Plan, submitted to the Department of Commerce In Response to The Presidential Spectrum Policy Reform Initiative, Feb. 2008.

[18] A. K. Rashid and Z. Shen, "A novel band-reject frequency selective surface with pseudo-elliptic response," IEEE Transactions on Antennas and Propagation, vol. 58, no. 4, pp. 1220-1226, Apr. 2010.

[19] B. Li and Z. Shen, "Three-dimensional bandpass frequency-selective structures with multiple transmission zeros," IEEE Transactions on Microwave Theory and Techniques, vol. 61, no. 10, pp. 3578-3589, Oct. 2013.

[20] S. Magnuson, "Military Learning to Share Congested Radio Spectrum," National Defense, Jan. 2017.

[21] Joint Electromagnetic Spectrum Operations, Joint Doctrine Note 3-16. (2016, Oct. 20). https://fas.org/irp/doddir/dod/jdn3_16.pdf

[22] J. Keller, "Air Force looking for power sources and antennas for future high-power microwave weapons," Military \& Aerospace Electronics, Apr. 2016. 
[23] X.-S. Yang, W.-Q. Deng, C.-S. Shen, and B.-Z. Wang, "Dual-polarized multi-layer patch antenna with pattern reconfigurable characteristic," in 2016 IEEE 5th AsiaPacific Conference on Antennas and Propagation, pp. 181-182, July 2016.

[24] N. Surittikul, "Pattern reconfiguraable printed antennas and time domain method of characteristic modes for antenna analysis and design," Ph.D. dissertation, The Ohio State University, 2006.

[25] G. R. DeJean, "Design of compact antennas in multilayer technology for wireless communications / WLAN applications," M.S. thesis, Georgia Institute of Technology, 2004.

[26] N. Amitay, V. Galindo, and C. P. Wu, Theory and analysis of phased array antennas. New York, NY, Wiley, 1972.

[27] A. W. Mathis and A. F. Peterson, "Efficient electromagnetic analysis of a doubly infinite array of rectangular apertures," IEEE Transactions on Microwave Theory and Techniques, vol. 46, no. 1, pp. 46-54, 1998.

[28] C. C. Chen, "Scattering by a two dimensional periodic array of conducting plates," IEEE Transactions, AP-18, pp. 660-665, 1970.

[29] J. P. Montgomery, "Scattering by an infinite periodic array of thin conductors on a dielectric sheet," IEEE Transactions, AP-23, pp. 70-75, 1975.

[30] J. L. Volakis, T. Ozdemir, and J. Gong, "Hybrid finite-element methodologies for antennas and scattering," IEEE Transactions on Antennas and Propagation, vol. 45, pp. 493-507, Mar. 1997.

[31] T. F. Eibert, K. Sertel, and J. L. Volakis, "Hybrid finite element modelling of conformal antenna and array structures utilizing fast integral methods," International Journal of Numerical Modeling: Electronic Networks, Devices and Fields, vol. 13, pp. 81-101, 2000.

[32] E. W. Lucas and T. W. Fontana, "A 3-D hybrid finite element/boundary element method for the unified radiation and scattering analysis of general infinite periodic arrays," IEEE Transactions on Antennas and Propagation, vol. 43, pp. 145-153, 1995.

[33] D. T. McGrath and V. P. Pyati, "Periodic structure analysis using a hybrid finite element method," Radio Science, vol. 31, pp. 1173-1179, 1996. 
[34] T. F. Eibert, J. L. Volakis, D. R. Wilton, and D. R. Jackson, "Hybrid FE/BI modeling of 3D doubly periodic structures utilizing triangular prismatic elements and a MPIE formulation accelerated by the Ewald transformation," IEEE Transactions on Antennas and Propagation, vol. 47, pp. 843-849, 1999.

[35] R. Remski, B. Gray, and L. Ma, "Frequency selective surfaces design," presented at Ansoft Empowering Profitability Workshop, Sept. 16, 2002.

[36] A. E. Williams, "A four-cavity elliptic waveguide filter," IEEE Transactions on Microwave Theory and Techniques, vol. 18, no. 12, pp. 1109-1114, Dec. 1970.

[37] L. Accatino, G. Bertin, and M. Mongiardo, "Elliptical cavity resonators for dualmode narrow-band filters," IEEE Transactions on Microwave Theory and Techniques, vol. 45, no. 12, pp. 2393-2401, Dec. 1997.

[38] A. K. Rashid, Z. Shen, and B. Li, "An elliptical bandpass frequency selective structure based on microstrip lines," IEEE Transactions on Antennas and Propagation, vol. 60, pp. 4661-4669, 2012.

[39] M. Yan, J. Wang, H. Ma, M. Feng, Y. Pang, S. Qu, J. Zhang, and L. Zheng, "A triband, highly selective, bandpass FSS using cascaded multilayer loop arrays," IEEE Transactions on Antennas and Propagation, vol. 64, no. 5, pp. 2046-2049, May 2016.

[40] K. Tao, B. Li, Y. Tang, and Q. Wu, "Multi-layer tri-band frequency selective surface using stepped- and uniformimpedance resonators," Electronics Letters, vol. 52, no. 8, pp. 583-585, Apr. 2016.

[41] B. Li and Z. Shen, "Dual-band bandpass frequency-selective structures with arbitrary band ratios," IEEE Transactions on Antennas and Propagation, vol. 62, no. 11, pp. 5504-5512, Nov. 2014.

[42] R. S. Anwar, L. Mao, and H. Ning, "Frequency Selective Surfaces: A Review," Applied Sciences, vol. 8, Sept. 2018.

[43] E. A. Parker, "The gentleman's guide to frequency selective surfaces," in 17th Q.M.W. Antenna Symposium, Apr. 1991.

[44] A. Mackay, B. Sanz-Izquierdo, E. A. Parker, "Evolution of frequency selective surfaces," FERMAT, vol. 2, pp. 1-7, 2014.

[45] B. Li and Z. Shen, "Wideband Bandpass Frequency Selective Structure Based on Periodic Array of Multi-Layer Strip Lines," in 2014 URSI General Assembly and Scientific Symposium, Aug. 2014. 
[46] I. G. Lee and I. P. Hong, "3D frequency selective surface for stable angle of incidence," Electronics Letters, vol. 50, no. 6, pp. 423-424, Mar. 2014.

[47] B. Liang and M. Bai, "Subwavelength three-dimensional frequency selective surface based on surface wave tunneling," Optics Express, vol. 24, no. 13, pp. 14697-14702, 2016.

[48] M.-J. Huang, M.-Y. Lv, J. Huang, and Z. Wu, "A new type of combined element multiband frequency selective surface," IEEE Transactions on Antennas and Propagation, vol. 57, no. 6, pp. 1798-1803, June 2009.

[49] M. Bouslama, M. Traii, T. A. Denidni, and A. Gharsallah, "Reconfigurable frequency selective surface for beam-switching applications," IET Microwaves, Antennas, \& Propagation, vol. 11, no. 1, pp. 69-74, Jun. 2016.

[50] M. Qu, M. Rao, S. Li, and L. Deng, "Tunable antenna radome based on graphene frequency selective surface," AIP Advances, vol. 7, 2017.

[51] G. I. Kiani, K. L. Ford, L. G. Olsson, K. P. Esselle, and C. J. Panagamuwa, "Switchable frequency selective surface for reconfigurable electromagnetic architecture of buildings," IEEE Transactions on Antennas and Propagation, vol. 58, pp. 581-584, Feb. 2010.

[52] B. Sanz-Izquierdo and E. A. Parker, "Dual polarized reconfigurable frequency selective surfaces," IEEE Transactions on Antennas and Propagation, vol. 62, pp. 764-771, Feb. 2014.

[53] K. ElMahgoub, F. Yang, and A. Z. Elsherbeni, "Design of novel reconfigurable frequency selective surfaces with two control techniques," Progress in Electromagnetics Research C, vol. 35, pp. 135-145, 2013.

[54] R. Sivasamy and M. Kanagasabai, "Novel reconfigurable 3-D frequency selective surface," IEEE Transactions on Components, Packaging, and Manufacturing Technology, vol. 7, no. 10, pp. 1678-1682, Oct. 2017.

[55] S. Ghosh and K. V. Srivastava, "Polarization-insensitive single-and broadband switchable absorber/reflector and its realization using a novel biasing technique," IEEE Transactions on Antennas and Propagation, vol. 64, pp. 3665-3670, Aug. 2016.

[56] T. Chang, R. J. Langley, and E. A. Parker, "Frequency selective surfaces on biased ferrite substrates," Electronics Letters, vol. 30, pp. 1193-1194, 1994. 
[57] E. A. Parker and S. Savia, "Active frequency selective surfaces with ferroelectric substrates," IEE Proc. - Microwaves, Antennas and Propagation, vol. 148, pp. 103-108, 2001.

[58] M. Li, B. Yu, and N. Behdad, "Liquid-tunable frequency selective surfaces," IEEE Microwave and Wireless Components Letters, vol. 20, no. 8, pp. 423-425, July 2010.

[59] E. Doumanis, G. Goussetis, R. Dickie, R. Cahill, P. Baine, M. Bain, V. Fusco, J. Encinar, and G. Toso, "Electronically reconfigurable liquid crystal based Mmwave polarization converter, IEEE Transactions on Antennas and Propagation, vol. 62, pp. 2302-2307, Apr. 2014.

[60] D. F. Mamedes, A. G. Neto1, J. C. Silva, and J. Bornemann, "Design of reconfigurable frequency-selective surfaces including the PIN diode threshold region," IET Microwaves, Antennas, \& Propagation, vol. 12, no. 9, pp. 1483-1486, Apr. 2018.

[61] L. Boccia, I. Russo, G. Amendola and G. Di Massa, "Tunable frequency-selective surfaces for beam-steering applications," Electronics Letters, vol. 45, no. 24, Nov. 2009.

[62] C. G. Christodoulou, Y. Tawk, S. A. Lane, and S. R. Erwin, "Reconfigurable antennas for wireless and space applications," Proc. IEEE, vol. 100, no. 7, Jul 2012.

[63] P. Kong, X. Yu, Z. Liu, K. Zhou, Y. He, L. Miao, and J. Jiang, "A novel tunable frequency selective surface absorber with dual-DOF for broadband applications, Optics Express, vol. 22, pp. 30217-30224, Nov. 2014.

[64] S. Ghosh and K. V. Srivastava, "Broadband polarization-insensitive tunable frequency selective surface for wideband shielding," IEEE Transactions on Electromagnetic Compatibility, vol. 60, pp. 166-172, Feb. 2018.

[65] A. Pourziad, S. Nikmehr, and H. Veladi, "A novel multi-state integrated RF MEMS switch for reconfigurable antennas applications," Progress in Electromagnetics Research, vol. 135, pp. 389-406, May 2013.

[66] R. Martinez-Lopez, J. Rodriguez-Cuevas, A. E. Martynyuk, and J. I. MartinezLopez, "An active ring slot with RF MEMS switchable radial stubs for reconfigurable frequency selective surface applications," Progress in Electromagnetics Research, vol. 128, pp. 419-440, June 2012. 
[67] X. G. Huang, Z. Shen, Q. Y. Feng, and B. Li, "Tunable 3-D bandpass frequencyselective structure with wide tuning range," IEEE Transactions on Antennas and Propagation, vol. 63, no. 7, pp. 3297-3301, Jul. 2015.

[68] S. N. Azemi, K. Ghorbani, and W. S. T. Rowe, "Mechanically tunable and reconfigurable FSS using spring loaded ring resonators," in Proc. European Microwave Conference, pp. 932-935, Oct. 2013.

[69] M. Bouslama, M. Traii, A. Gharsallah, and T. A. Denidni, "Reconfigurable dualband 3D frequency selective surface unit-cell," in Proc. IEEE International Symposium on Antennas and Propagation, pp. 1264-1265, Jul. 2015.

[70] D. Ferreira, I. Cuiñas, R. F. S. Caldeirinha, and T. R. Fernandes, "3-D Mechanically Tunable Square Slot FSS," IEEE Transactions on Antennas and Propagation, vol. 65, no. 1, pp. 242-250, Jan. 2017.

[71] Z. Chen, Y. Wang, X. Wang, R. Wang, and L. Hao, "Reconfigurable frequency selective surface radome for tunable antenna RCS reduction," in 2017 International Applied Computational Electromagnetics Society Symposium, Aug. 2017.

[72] J. Roberts, K. L. Ford, and J. M. Rigelsford, "Secure electromagnetic buildings using slow phase-switching frequency-selective surfaces," IEEE Transactions on Antennas and Propagation, vol. 64, no. 1, pp. 251-261, Jan. 2016.

[73] C. H. Cox and E. I. Ackerman, "Photonics for simultaneous transmit and receive," Microwave Symposium Digest, 2011 IEEE MTT-S International, pp.1-4, June 2011.

[74] H. Yang, X. Cao, F. Yang, J. Gao, S. Xu, M. Li, X. Chen, Y. Zhao, Y. Zheng, and $\mathrm{S}$. Li, "A programmable metasurface with dynamic polarization, scattering and focusing control," Scientific Reports, Oct. 2016.

[75] C. Fernandez-Prades, P. Closas, and J. Arribas, "Implementation of digital beamforming in GNSS receivers," Proceedings of the European Workshop on GNSS Signals and Signal Processing, 2009.

[76] A. K. Rashid, B. Li, and Z. Shen, "An overview of three-dimensional frequencyselective structures," IEEE Antennas and Propagation Magazine, vol. 56, no. 3, pp. 43-67, June 2014.

[77] L. Zhu, $\mathrm{H}$. Bu, and $\mathrm{K}$. Wu, "Aperture compensation technique for innovative design of ultra-broadband microstrip bandpass filter," IEEE MTT-S International Microwave Symposium Digest, vol. 1, pp. 315-318, 2000. 
[78] W. Menzel, L. Zhu, K. Wu, and F. Bogelsack, "On the design of novel compact broad-band planar filters," IEEE Transactions on Microwave Theory and Techniques, vol. 51, pp. 364-370, 2003.

[79] C. A. Balanis, Antenna Theory: Analysis and Design. New York, NY, Wiley, 1997.

[80] H. So, A. Ando, T. Seki, M. Kawashima, and T. Sugiyama, "Directional multi-band antenna employing frequency selective surfaces," Electronics Letters, vol. 49, pp. 243-245, 2013.

[81] A. K. Palange, A. Sonker, and S. S. Yadav, "Designing of multiband frequency selective surfaces," in 2016 International Conference on Communication and Signal Processing, Apr. 2016

[82] B. Rahmati and H. R. Hassani, "Multiband metallic frequency selective surface with wide range of band ratio," IEEE Transactions on Antennas and Propagation, vol. 63, no. 8, pp. 3747-3753, Aug. 2015.

[83] A. L. Campos, T. L. Silva, and A. G. Neto, "Multiband frequency selective surfaces with simple modification of a rectangular patch element," Microwave and Optical Technology Letters, Sept. 2013.

[84] T. Edwards, Foundations for Microstrip Circuit Design. New York, NY, Wiley, 1991.

[85] H. Jaafar, K. S. Beh, N. A. Yunus, W. Z. Hasan, S. Shafie, and O. Sidek, "A comprehensive study on RF MEMS switch," Microsystem Technologies, vol. 20, no. 12, pp. 2109-2121, Aug. 2014.

[86] B. Manz, "The high-performance RF MEMS switch has arrived," Electronic Design, July 2018.

[87] Z. Sipus, M. Bosiljevac, and S. Skokic, "Analysis of curved frequency selective surfaces", in Proc. EuCAP 2007, Dec. 2007.

[88] O. Manoochehri, S. Abbasiniazare, A. Torabi, and K. Forooraghi, "A second-order BPF using a miniaturized-element frequency selective surface," Progress in Electromagnetics Research C, vol. 31, pp. 229-240, 2012. 